\title{
Noncommutative instantons from twisted conformal symmetries
}

\author{
Giovanni Landi ${ }^{1}$, Walter D. van Suijlekom ${ }^{2}$ \\ ${ }^{1}$ Dipartimento di Matematica e Informatica, Università di Trieste \\ Via A. Valerio 12/1, I-34127 Trieste, Italy \\ and INFN, Sezione di Trieste, Trieste, Italy \\ landi@univ.trieste.it \\ ${ }^{2}$ Max Planck Institute for Mathematics \\ Vivatsgasse 7, D-53111 Bonn, Germany \\ waltervs@mpim-bonn .mpg . de
}

31 July 2006

\begin{abstract}
We construct a five-parameter family of gauge-nonequivalent $S U(2)$ instantons on a noncommutative four sphere $S_{\theta}^{4}$ and of topological charge equal to 1 . These instantons are critical points of a gauge functional and satisfy self-duality equations with respect to a Hodge star operator on forms on $S_{\theta}^{4}$. They are obtained by acting with a twisted conformal symmetry on a basic instanton canonically associated with a noncommutative instanton bundle on the sphere. A completeness argument for this family is obtained by means of index theorems. The dimension of the "tangent space" to the moduli space is computed as the index of a twisted Dirac operator and turns out to be equal to five, a number that survives deformation.
\end{abstract}




\section{Contents}

1 Introduction 3

2 Connections and gauge transformations 4

2.1 Connections on modules . . . . . . . . . . . . . . . . . 4

2.2 Gauge transformations . . . . . . . . . . . . . . . 8

3 Toric noncommutative manifolds $M_{\theta}$

3.1 Deforming a torus action . . . . . . . . . . . . . . . . . . . 9

3.2 The manifold $M_{\theta}$ as a fixed point algebra . . . . . . . . . 12

3.3 Vector bundles on $M_{\theta} \ldots \ldots \ldots \ldots \ldots$. . . . . . . . . . . . . . . . . . . . . . . . .

3.4 Differential calculus on $M_{\theta} \ldots \ldots \ldots \ldots \ldots$

4 Gauge theory on the sphere $S_{\theta}^{4} \quad \mathbf{1 6}$

4.1 The principal fibration $S_{\theta^{\prime}}^{7} \rightarrow S_{\theta}^{4} \ldots \ldots \ldots \ldots \ldots \ldots$

4.2 Associated bundles . . . . . . . . . . . . . . . . . . . . 19

4.3 Yang-Mills theory on $S_{\theta}^{4} \ldots \ldots \ldots \ldots \ldots \ldots$

5 Construction of $\mathrm{SU}(2)$-instantons on $S_{\theta}^{4} \quad 25$

5.1 The basic instanton . . . . . . . . . . . . . . . . . 25

5.2 Twisted infinitesimal symmetries . . . . . . . . . . . . . . . . . 27

5.3 Twisted conformal transformations . . . . . . . . . . . . . . 31

5.4 Local expressions . . . . . . . . . . . . . . . . . . . . . . . . . . . . . . . . . . . .

5.5 Moduli space of instantons . . . . . . . . . . . . . . . . . . 37

5.6 Dirac operator associated to the complex . . . . . . . . . . 39

6 Towards Yang-Mills theory on $M_{\theta} \quad 40$

A Local index formula 43 


\section{Introduction}

The importance of Yang-Mills instantons in physics and mathematics needs not be stressed. They have played a central role since their first appearance [7] and are most elegantly described via the so-called ADHM construction [5, 4]. The generalization in [30] of this method for instantons on a noncommutative space $\mathbb{R}^{4}$ has found several important applications notably in brane and superconformal theories.

Toric noncommutative manifolds $M_{\Theta}$ were constructed and studied in [15]. One starts with any (Riemannian spin) manifold $M$ carrying a torus action and then deforms the torus to a noncommutative one governed by a real antisymmetric matrix $\Theta$ of deformation parameters. The starting example of [15] - the archetype of all these deformations - was a four dimensional sphere $S_{\theta}^{4}$, which came with a natural noncommutative instanton bundle endowed with a natural connection. At the classical value of the deformation parameter, $\theta=0$, the bundle and the connection reduces to the one of [7]. The present sphere $S_{\theta}^{4}$ can be thought of [14] as a one point compactification of a noncommutative $\mathbb{R}_{\theta}^{4}$ which is structurally different from the one considered in [30].

In [27] this basic noncommutative instanton was put in the context of an $\mathrm{SU}(2)$ noncommutative principal fibration $S_{\theta^{\prime}}^{7} \rightarrow S_{\theta}^{4}$ over $S_{\theta}^{4}$. In the present paper, we continue the analysis and consider it in the setting of a noncommutative Yang-Mills theory. We then construct a five-parameter family of (infinitesimal) gauge-nonequivalent instantons, by acting with twisted conformal symmetries on the basic instanton. All these instantons will be gauge configurations satisfying self-duality equations - with a suitable defined Hodge $*_{\theta}$-operator on forms $\Omega\left(S_{\theta}^{4}\right)$ - and will have a "topological charge" of value 1 . A completeness argument on the family of instantons is provided by index theoretical arguments, similar to the one in [6] for undeformed instantons on $S^{4}$. The dimension of the "tangent" of the moduli space can be computed as the index of a twisted Dirac operator which turns out to be equal to its classical value that is five. The twisting of the conformal symmetry is implemented with a twist of Drinfel'd type [18, 19] - in fact, explicitly constructed by Reshetikhin [31] - and gives rise to a deformed Hopf algebra $\mathrm{U}_{\theta}(s o(5,1))$. That these are conformal infinitesimal transformations is stressed by the fact that the Hopf algebra $\mathrm{U}_{\theta}(s o(5,1))$ leaves the Hodge $*_{\theta}$-structure of $\Omega\left(S_{\theta}^{4}\right)$ invariant.

The paper is organized as follows. In Section 2 we recall the setting of gauge theories (connections) and gauge transformations on finite projective modules (the substitute for vector bundles) over algebras (the substitute for spaces). The main objective is to implement a Bianchi identity that will be crucial later on for the self-duality equations.

Section 3 deals with toric noncommutative manifolds. These were indeed named isospectral deformations in that they can be endowed with the structure of a noncommutative Riemannian spin manifold via a spectral triple $\left(C^{\infty}\left(M_{\theta}\right), D, \mathcal{H}\right)$ with the properties of [13]. For this class of examples, the Dirac operator $D$ is the classical one and $\mathcal{H}=L^{2}(M, \mathcal{S})$ is the usual Hilbert space of spinors on which the algebra $C^{\infty}\left(M_{\theta}\right)$ acts in a twisted manner. Thus one twists the algebra and its representation while keeping the geometry unchanged. The resulting noncommutative geometry is isospectral and all spectral properties are preserved including the dimension. Both the algebra and its action on spinors can be given via a "star-type" product.

In Section 4 we specialize to gauge theories on the sphere $S_{\theta}^{4}$ and introduce a YangMills action functional, from which we derive field equations (equations for critical points), 
as well as a topological action functional whose absolute value gives a lower bound for the Yang-Mills action.

The heart of the paper is Section 5 were we explicitly construct instantons. As usual, these are gauge configurations which are solutions of (anti)self-duality equations and realize absolute minima of the Yang-Mills functional. We start from a basic instanton which is shown to be invariant under twisted orthogonal transformations in $\mathrm{U}_{\theta}(s o(5))$. We then perturb it by the action of conformal operators in $\mathrm{U}_{\theta}(s o(5,1))-\mathrm{U}_{\theta}(s o(5))$ producing a five parameter family of new, not gauge equivalent instantons. A completeness argument is obtained by using an index theorem to compute the dimension of the tangent space of the moduli space of instantons on $S_{\theta}^{4}$, which is shown to be just five. The relevant material from noncommutative index theory is recalled in the appendix.

Section 6] sketches a general scheme for gauge theories on four dimensional toric noncommutative manifolds.

\section{Connections and gauge transformations}

We first review the notion of a (gauge) connection on a (finite projective) module $\mathcal{E}$ over an algebra $\mathcal{A}$ with respect to a given calculus; we take a right module structure. Also, we recall gauge transformations in this setting. We refer to [12] for more details (see also [24]).

\subsection{Connections on modules}

Let us suppose we have an algebra $\mathcal{A}$ with a differential calculus $\left(\Omega \mathcal{A}=\oplus_{p} \Omega^{p} \mathcal{A}, \mathrm{d}\right)$. A connection on the right $\mathcal{A}$-module $\mathcal{E}$ is a $\mathbb{C}$-linear map

$$
\nabla: \mathcal{E} \otimes_{\mathcal{A}} \Omega^{p} \mathcal{A} \longrightarrow \mathcal{E} \otimes_{\mathcal{A}} \Omega^{p+1} \mathcal{A}
$$

defined for any $p \geq 0$, and satisfying the Leibniz rule

$$
\nabla(\omega \rho)=(\nabla \omega) \rho+(-1)^{p} \omega \mathrm{d} \rho, \quad \forall \omega \in \mathcal{E} \otimes_{\mathcal{A}} \Omega^{p} \mathcal{A}, \rho \in \Omega \mathcal{A} .
$$

A connection is completely determined by its restriction

$$
\nabla: \mathcal{E} \rightarrow \mathcal{E} \otimes_{\mathcal{A}} \Omega^{1} \mathcal{A}
$$

which satisfies

$$
\nabla(\eta a)=(\nabla \eta) a+\eta \otimes_{\mathcal{A}} \mathrm{d} a, \quad \forall \eta \in \mathcal{E}, a \in \mathcal{A},
$$

and which is extended to all of $\mathcal{E} \otimes_{\mathcal{A}} \Omega^{p} \mathcal{A}$ using Leibniz rule. It is the latter rule that implies the $\Omega \mathcal{A}$-linearity of the composition,

$$
\nabla^{2}=\nabla \circ \nabla: \mathcal{E} \otimes_{\mathcal{A}} \Omega^{p} \mathcal{A} \longrightarrow \mathcal{E} \otimes_{\mathcal{A}} \Omega^{p+2} \mathcal{A}
$$

Indeed, for any $\omega \in \mathcal{E} \otimes_{\mathcal{A}} \Omega^{p} \mathcal{A}$ and $\rho \in \Omega \mathcal{A}$ it follows that $\nabla^{2}(\omega \rho)=\nabla((\nabla \omega) \rho+$ $\left.(-1)^{p} \omega \mathrm{d} \rho\right)=\left(\nabla^{2} \omega\right) \rho+(-1)^{p+1}(\nabla \omega) \mathrm{d} \rho+(-1)^{p}(\nabla \omega) \mathrm{d} \rho+\omega \mathrm{d}^{2} \rho=\left(\nabla^{2} \omega\right) \rho$. The restriction of $\nabla^{2}$ to $\mathcal{E}$ is the curvature

$$
F: \mathcal{E} \rightarrow \mathcal{E} \otimes_{\mathcal{A}} \Omega^{2} \mathcal{A}
$$


of the connection. It is $\mathcal{A}$-linear, $F(\eta a)=F(\eta) a$ for any $\eta \in \mathcal{E}, a \in \mathcal{A}$, and satisfies

$$
\nabla^{2}\left(\eta \otimes_{\mathcal{A}} \rho\right)=F(\eta) \rho, \quad \forall \eta \in \mathcal{E}, \rho \in \Omega \mathcal{A} .
$$

Thus, $F \in \operatorname{Hom}_{\mathcal{A}}\left(\mathcal{E}, \mathcal{E} \otimes_{\mathcal{A}} \Omega^{2} \mathcal{A}\right)$, the latter being the collection of (right) $\mathcal{A}$-linear homomorphisms from $\mathcal{E}$ to $\mathcal{E} \otimes_{\mathcal{A}} \Omega^{2} \mathcal{A}$ (an alternative notation for this collection that is used in the literature, is $\left.\operatorname{End}_{\mathcal{A}}\left(\mathcal{E}, \mathcal{E} \otimes_{\mathcal{A}} \Omega^{2} \mathcal{A}\right)\right)$.

In order to have the notion of a Bianchi identity we need some generalization. Let $\operatorname{End}_{\Omega \mathcal{A}}\left(\mathcal{E} \otimes_{\mathcal{A}} \Omega \mathcal{A}\right)$ be the collection of all $\Omega \mathcal{A}$-linear endomorphisms of $\mathcal{E} \otimes_{\mathcal{A}} \Omega \mathcal{A}$. It is an algebra under composition. The curvature $F$ can be thought of as an element of $\operatorname{End}_{\Omega \mathcal{A}}\left(\mathcal{E} \otimes_{\mathcal{A}} \Omega \mathcal{A}\right)$. There is a map

$$
\begin{aligned}
{[\nabla, \cdot] } & : \operatorname{End}_{\Omega \mathcal{A}}\left(\mathcal{E} \otimes_{\mathcal{A}} \Omega \mathcal{A}\right) \longrightarrow \operatorname{End}_{\Omega \mathcal{A}}\left(\mathcal{E} \otimes_{\mathcal{A}} \Omega \mathcal{A}\right), \\
{[\nabla, T] } & :=\nabla \circ T-(-1)^{|T|} T \circ \nabla
\end{aligned}
$$

where $|T|$ denotes the degree of $T$ with respect to the $\mathbb{Z}^{2}$-grading of $\Omega \mathcal{A}$. Indeed, for any $\omega \in \mathcal{E} \otimes_{\mathcal{A}} \Omega^{p} \mathcal{A}$ and $\rho \in \Omega \mathcal{A}$, it follows that

$$
\begin{aligned}
{[\nabla, T](\omega \rho) } & =\nabla(T(\omega \rho))-(-1)^{|T|} T(\nabla(\omega \rho)) \\
& =\nabla(T(\omega) \rho)-(-1)^{|T|} T\left((\nabla \omega) \rho+(-1)^{p} \omega \mathrm{d} \rho\right) \\
& =(\nabla(T(\omega))) \rho+(-1)^{p+|T|} T(\omega) \mathrm{d} \rho-(-1)^{|T|} T(\nabla \omega) \rho-(-1)^{p+|T|} T(\omega) \mathrm{d} \rho \\
& =\left(\nabla(T(\omega))-(-1)^{|T|} T(\nabla \omega)\right) \rho=([\nabla, T](\omega)) \rho,
\end{aligned}
$$

and the map in (2.5) is well-defined. It is straightforwardly checked that $[\nabla, \cdot]$ is a graded derivation for the algebra $\operatorname{End}_{\Omega \mathcal{A}}\left(\mathcal{E} \otimes_{\mathcal{A}} \Omega \mathcal{A}\right)$,

$$
[\nabla, S \circ T]=[\nabla, S] \circ T+(-1)^{|S|} S \circ[\nabla, T] .
$$

Proposition 1. The curvature F satisfies the Bianchi identity,

$$
[\nabla, F]=0 .
$$

Proof. Since $F$ is an even element in $\operatorname{End}_{\Omega \mathcal{A}}\left(\mathcal{E} \otimes_{\mathcal{A}} \Omega \mathcal{A}\right)$, the map $[\nabla, F]$ makes sense. Furthermore,

$$
[\nabla, F]=\nabla \circ \nabla^{2}-\nabla^{2} \circ \nabla=\nabla^{3}-\nabla^{3}=0 .
$$

In Section II.2 of [11], such a Bianchi identity was implicitly used in the construction of a so-called canonical cycle from a connection on a finite projective $\mathcal{A}$-module $\mathcal{E}$.

Connections always exist on a projective module. On the module $\mathcal{E}=\mathbb{C}^{N} \otimes_{\mathbb{C}} \mathcal{A} \simeq \mathcal{A}^{N}$, which is free, a connection is given by the operator

$$
\nabla_{0}=\mathbb{I} \otimes \mathrm{d}: \mathbb{C}^{N} \otimes_{\mathbb{C}} \Omega^{p} \mathcal{A} \longrightarrow \mathbb{C}^{N} \otimes_{\mathbb{C}} \Omega^{p+1} \mathcal{A}
$$

With the canonical identification $\mathbb{C}^{N} \otimes_{\mathbb{C}} \Omega \mathcal{A}=\left(\mathbb{C}^{N} \otimes_{\mathbb{C}} \mathcal{A}\right) \otimes_{\mathcal{A}} \Omega \mathcal{A} \simeq(\Omega \mathcal{A})^{N}$, one thinks of $\nabla_{0}$ as acting on $(\Omega \mathcal{A})^{N}$ as the operator $\nabla_{0}=(\mathrm{d}, \mathrm{d}, \cdots, \mathrm{d})(N$-times $)$. Next, take a projective module $\mathcal{E}$ with inclusion map, $\lambda: \mathcal{E} \rightarrow \mathcal{A}^{N}$, which identifies $\mathcal{E}$ as a direct summand of the free module $\mathcal{A}^{N}$ and idempotent $p: \mathcal{A}^{N} \rightarrow \mathcal{E}$ which allows one to identify 
$\mathcal{E}=p \mathcal{A}^{N}$. Using these maps and their natural extensions to $\mathcal{E}$-valued forms, a connection $\nabla_{0}$ on $\mathcal{E}$ (called Levi-Civita or Grassmann) is the composition,

$$
\mathcal{E} \otimes_{\mathcal{A}} \Omega^{p} \mathcal{A} \stackrel{\lambda}{\longrightarrow} \mathbb{C}^{N} \otimes_{\mathbb{C}} \Omega^{p} \mathcal{A} \stackrel{\mathbb{I} \otimes \mathrm{d}}{\longrightarrow} \mathbb{C}^{N} \otimes_{\mathbb{C}} \Omega^{p+1} \mathcal{A} \stackrel{p}{\longrightarrow} \mathcal{E} \otimes_{\mathcal{A}} \Omega^{p+1} \mathcal{A}
$$

that is

$$
\nabla_{0}=p \circ(\mathbb{I} \otimes \mathrm{d}) \circ \lambda .
$$

One indicates it simply by $\nabla_{0}=p \mathrm{~d}$. The space $C(\mathcal{E})$ of all connections on $\mathcal{E}$ is an affine space modeled on $\operatorname{Hom}_{\mathcal{A}}\left(\mathcal{E}, \mathcal{E} \otimes_{\mathcal{A}} \Omega^{1} \mathcal{A}\right)$. Indeed, if $\nabla_{1}, \nabla_{2}$ are two connections on $\mathcal{E}$, their difference is $\mathcal{A}$-linear,

$$
\left(\nabla_{1}-\nabla_{2}\right)(\eta a)=\left(\left(\nabla_{1}-\nabla_{2}\right)(\eta)\right) a, \quad \forall \eta \in \mathcal{E}, a \in \mathcal{A},
$$

so that $\nabla_{1}-\nabla_{2} \in \operatorname{Hom}_{\mathcal{A}}\left(\mathcal{E}, \mathcal{E} \otimes_{\mathcal{A}} \Omega^{1} \mathcal{A}\right)$. Thus, any connection can be written as

$$
\nabla=p \mathrm{~d}+\alpha
$$

where $\alpha$ is any element in $\operatorname{Hom}_{\mathcal{A}}\left(\mathcal{E}, \mathcal{E} \otimes_{\mathcal{A}} \Omega^{1} \mathcal{A}\right)$. The "matrix of 1 -forms" $\alpha$ as in (2.9) is called the gauge potential of the connection $\nabla$. The corresponding curvature $F$ of $\nabla$ is

$$
F=p \mathrm{~d} p \mathrm{~d} p+p \mathrm{~d} \alpha+\alpha^{2}
$$

Next, let the algebra $\mathcal{A}$ have an involution *; it is extended to the whole of $\Omega \mathcal{A}$ by the requirement $(\mathrm{d} a)^{*}=\mathrm{d} a^{*}$ for any $a \in \mathcal{A}$. A Hermitian structure on the module $\mathcal{E}$ is a $\operatorname{map}\langle\cdot, \cdot\rangle: \mathcal{E} \times \mathcal{E} \rightarrow \mathcal{A}$ with the properties

$$
\begin{aligned}
& \langle\eta, \xi a\rangle=\langle\xi, \eta\rangle a, \quad\langle\eta, \xi\rangle^{*}=\langle\xi, \eta\rangle, \\
& \langle\eta, \eta\rangle \geq 0, \quad\langle\eta, \eta\rangle=0 \Longleftrightarrow \eta=0,
\end{aligned}
$$

for any $\eta, \xi \in \mathcal{E}$ and $a \in \mathcal{A}$ (an element $a \in \mathcal{A}$ is positive if it is of the form $a=b^{*} b$ for some $b \in \mathcal{A}$ ). We shall also require the Hermitian structure to be self-dual, i.e. every right $\mathcal{A}$-module homomorphism $\phi: \mathcal{E} \rightarrow \mathcal{A}$ is represented by an element of $\eta \in \mathcal{E}$, by the assignment $\phi(\cdot)=\langle\eta, \cdot\rangle$, the latter having the correct properties by the first of (2.11).

The Hermitian structure is naturally extended to an $\Omega \mathcal{A}$-valued linear map on the product $\mathcal{E} \otimes_{\mathcal{A}} \Omega \mathcal{A} \times \mathcal{E} \otimes_{\mathcal{A}} \Omega \mathcal{A}$ by

$$
\left\langle\eta \otimes_{\mathcal{A}} \omega, \xi \otimes_{\mathcal{A}} \rho\right\rangle=(-1)^{|\eta||\omega|} \omega^{*}\langle\eta, \xi\rangle \rho, \quad \forall \eta, \xi \in \mathcal{E} \otimes_{\mathcal{A}} \Omega \mathcal{A}, \omega, \rho \in \Omega \mathcal{A} .
$$

A connection $\nabla$ on $\mathcal{E}$ and a Hermitian structure $\langle\cdot, \cdot\rangle$ on $\mathcal{E}$ are said to be compatible if the following condition is satisfied [12],

$$
\langle\nabla \eta, \xi\rangle+\langle\eta, \nabla \xi\rangle=\mathrm{d}\langle\eta, \xi\rangle, \quad \forall \eta, \xi \in \mathcal{E}
$$

It follows directly from the Leibniz rule and (2.12) that this extends to

$$
\langle\nabla \eta, \xi\rangle+(-1)^{|\eta|}\langle\eta, \nabla \xi\rangle=\mathrm{d}\langle\eta, \xi\rangle, \quad \forall \eta, \xi \in \mathcal{E} \otimes_{\mathcal{A}} \Omega \mathcal{A} .
$$


On the free module $\mathcal{A}^{N}$ there is a canonical Hermitian structure given by

$$
\langle\eta, \xi\rangle=\sum_{j=1}^{N} \eta_{j}^{*} \xi_{j},
$$

with $\eta=\left(\eta_{1}, \cdots, \eta_{N}\right)$ and $\eta=\left(\eta_{1}, \cdots, \eta_{N}\right)$ any two elements of $\mathcal{A}^{N}$.

Under suitable regularity conditions on the algebra $\mathcal{A}$ all Hermitian structures on a given finite projective module $\mathcal{E}$ over $\mathcal{A}$ are isomorphic to each other and are obtained from the canonical structure (2.15) on $\mathcal{A}^{N}$ by restriction [12, II.1]. Moreover, if $\mathcal{E}=p \mathcal{A}^{N}$, then $p$ is self-adjoint: $p=p^{*}$, with $p^{*}$ obtained by the composition of the involution ${ }^{*}$ in the algebra $\mathcal{A}$ with the usual matrix transposition. The Grassmann connection (2.8) is easily seen to be compatible with this Hermitian structure,

$$
\mathrm{d}\langle\eta, \xi\rangle=\left\langle\nabla_{0} \eta, \xi\right\rangle+\left\langle\eta, \nabla_{0} \xi\right\rangle .
$$

For a general connection (2.9), the compatibility with the Hermitian structure reduces to

$$
\langle\alpha \eta, \xi\rangle+\langle\eta, \alpha \xi\rangle=0, \quad \forall \eta, \xi \in \mathcal{E}
$$

which just says that the gauge potential is skew-hermitian,

$$
\alpha^{*}=-\alpha .
$$

We still use the symbol $C(\mathcal{E})$ to denote the space of compatible connections on $\mathcal{E}$.

Let $\operatorname{End}_{\Omega \mathcal{A}}^{s}\left(\mathcal{E} \otimes_{\mathcal{A}} \Omega \mathcal{A}\right)$ denote the space of elements $T$ in $\operatorname{End}_{\Omega \mathcal{A}}\left(\mathcal{E} \otimes_{\mathcal{A}} \Omega \mathcal{A}\right)$ which are skew-hermitian with respect to the Hermitian structure (2.12), i.e. satisfying

$$
\langle T \eta, \xi\rangle+\langle\eta, T \xi\rangle=0, \quad \forall \eta, \xi \in \mathcal{E}
$$

Proposition 2. The map $[\nabla$, · $]$ in (2.5) restricts to $\operatorname{End}_{\Omega \mathcal{A}}^{s}\left(\mathcal{E} \otimes_{\mathcal{A}} \Omega \mathcal{A}\right)$ as a derivation

$$
[\nabla, \cdot]: \operatorname{End}_{\Omega \mathcal{A}}^{s}\left(\mathcal{E} \otimes_{\mathcal{A}} \Omega \mathcal{A}\right) \longrightarrow \operatorname{End}_{\Omega \mathcal{A}}^{s}\left(\mathcal{E} \otimes_{\mathcal{A}} \Omega \mathcal{A}\right)
$$

Proof. Let $T \in \operatorname{End}_{\Omega \mathcal{A}}^{s}\left(\mathcal{E} \otimes_{\mathcal{A}} \Omega \mathcal{A}\right)$ be of order $|T|$; it then satisfies

$$
\langle T \eta, \xi\rangle+(-1)^{|\eta||T|}\langle\eta, T \xi\rangle=0,
$$

for $\eta, \xi \in \mathcal{E} \otimes_{\mathcal{A}} \Omega \mathcal{A}$. Since $[\nabla, T]$ is $\Omega \mathcal{A}$-linear, it is enough to show that

$$
\langle[\nabla, T] \eta, \xi\rangle+\langle\eta,[\nabla, T] \xi\rangle=0, \quad \forall \eta, \xi \in \mathcal{E} .
$$

This follows from equations (2.21) and (2.14),

$$
\begin{aligned}
\langle[\nabla, T] \eta, \xi\rangle+\langle\eta,[\nabla, T] \xi\rangle & =\langle\nabla T \eta, \xi\rangle-(-1)^{|T|}\langle T \nabla \eta, \xi\rangle+\langle\eta, \nabla T \xi\rangle-(-1)^{|T|}\langle\eta, T \nabla \xi\rangle \\
& =\langle\nabla T \eta, \xi\rangle-\langle\nabla \eta, T \xi\rangle+\langle\eta, \nabla T \xi\rangle-(-1)^{|T|}\langle T \eta, \nabla \xi\rangle \\
& =\mathrm{d}(\langle T \eta, \xi\rangle+\langle\eta, T \xi\rangle)=0 .
\end{aligned}
$$




\subsection{Gauge transformations}

We now add the additional requirement that the algebra $\mathcal{A}$ is a Fréchet algebra and that $\mathcal{E}$ a right Fréchet module. That is, both $\mathcal{A}$ and $\mathcal{E}$ are complete in the topology defined by a family of seminorms $\|\cdot\|_{i}$ such that the following condition is satisfied: for all $j$ there exists a constant $c_{j}$ and an index $k$ such that

$$
\|\eta a\|_{j} \leq c_{j}\|\eta\|_{k}\|a\|_{k}
$$

The collection $\operatorname{End}_{\mathcal{A}}(\mathcal{E})$ of all $\mathcal{A}$-linear maps is an algebra with involution; its elements are also called endomorphisms of $\mathcal{E}$. It becomes a Fréchet algebra with the following family of seminorms: for $T \in \operatorname{End}_{\mathcal{A}}(\mathcal{E})$,

$$
\|T\|_{i}=\sup _{\eta}\left\{\|T \eta\|_{i}:\|\eta\|_{i} \leq 1\right\}
$$

Since we are taking a self-dual Hermitian structure (see the discussion after (2.11)), any $T \in \operatorname{End}_{\mathcal{A}}(\mathcal{E})$ is adjointable, that is it admits an adjoint, an $\mathcal{A}$-linear map $T^{*}: \mathcal{E} \rightarrow \mathcal{E}$ such that

$$
\left\langle T^{*} \eta, \xi\right\rangle=\langle\eta, T \xi\rangle, \quad \forall \eta, \xi \in \mathcal{E} .
$$

The group $\mathcal{U}(\mathcal{E})$ of unitary endomorphisms of $\mathcal{E}$ is given by

$$
\mathcal{U}(\mathcal{E}):=\left\{u \in \operatorname{End}_{\mathcal{A}}(\mathcal{E}) \mid u u^{*}=u^{*} u=\operatorname{id}_{\mathcal{E}}\right\}
$$

This group plays the role of the infinite dimensional group of gauge transformations. It naturally acts on compatible connections by

$$
(u, \nabla) \mapsto \nabla^{u}:=u^{*} \nabla u, \quad \forall u \in \mathcal{U}(\mathcal{E}), \quad \nabla \in C(\mathcal{E}),
$$

where $u^{*}$ is really $u^{*} \otimes \operatorname{id}_{\Omega \mathcal{A}}$; this will always be understood in the following. Then the curvature transforms in a covariant way

$$
(u, F) \mapsto F^{u}=u^{*} F u,
$$

since, evidently, $F^{u}=\left(\nabla^{u}\right)^{2}=u^{*} \nabla u u^{*} \nabla u^{*}=u^{*} \nabla^{2} u=u^{*} F u$.

As for the gauge potential, one has the usual affine transformation,

$$
(u, \alpha) \mapsto \alpha^{u}:=u^{*} p \mathrm{~d} u+u^{*} \alpha u .
$$

Indeed, $\nabla^{u}(\eta)=u^{*}(p \mathrm{~d}+\alpha) u \eta=u^{*} p \mathrm{~d}(u \eta)+u^{*} \alpha u \eta=u^{*} p u \mathrm{~d} \eta+u^{*} p(\mathrm{~d} u) \eta+u^{*} \alpha u \eta=$ $p \mathrm{~d} \eta+\left(u^{*} p \mathrm{~d} u+u^{*} \alpha u\right) \eta$ for any $\eta \in \mathcal{E}$, which yields (2.27) for the transformed potential.

The "tangent vectors" to the gauge group $\mathcal{U}(\mathcal{E})$ constitute the vector space of infinitesimal gauge transformations. Suppose $\left\{u_{t}\right\}_{t \in \mathbb{R}}$ is a differentiable family of elements in $\operatorname{End}_{\mathcal{A}}(\mathcal{E})$ (in the topology defined by the above sup-norms) and define $X:=\left(\partial u_{t} / \partial t\right)_{t=0}$. Unitarity of $u_{t}$ then induces that $X=-X^{*}$. In other words, for $u_{t}$ to be a gauge transformation, $X$ should be a skew-hermitian endomorphisms of $\mathcal{E}$. In this way, we understand $\operatorname{End}_{\mathcal{A}}^{s}(\mathcal{E})$ as the collection of infinitesimal gauge transformations. It is a real vector space whose complexification $\operatorname{End}_{\mathcal{A}}^{s}(\mathcal{E}) \otimes_{\mathbb{R}} \mathbb{C}$ can be identified with $\operatorname{End}_{\mathcal{A}}(\mathcal{E})$. 
Infinitesimal gauge transformations act on a connection in a natural way. Let the gauge transformation $u_{t}$, with $X=\left(\partial u_{t} / \partial t\right)_{t=0}$, act on $\nabla$ as in (2.25). From the fact that $\left(\partial\left(u_{t} \nabla u_{t}^{*}\right) / \partial t\right)_{t=0}=[\nabla, X]$, we conclude that an element $X \in \operatorname{End}_{\mathcal{A}}^{s}(\mathcal{E})$ acts infinitesimally on a connection $\nabla$ by the addition of $[\nabla, X]$,

$$
(X, \nabla) \mapsto \nabla^{X}=\nabla+t[\nabla, X]+\mathcal{O}\left(t^{2}\right), \quad \forall X \in \operatorname{End}_{\mathcal{A}}^{s}(\mathcal{E}), \quad \nabla \in C(\mathcal{E}) .
$$

As a consequence, for the transformed curvature one finds

$$
(X, F) \mapsto F^{X}=F+t[F, X]+\mathcal{O}\left(t^{2}\right),
$$

since $F^{X}=(\nabla+t[\nabla, X]) \circ(\nabla+t[\nabla, X])=\nabla^{2}+t\left[\nabla^{2}, X\right]+\mathcal{O}\left(t^{2}\right)$.

\section{Toric noncommutative manifolds $M_{\theta}$}

We start by recalling the general construction of toric noncommutative manifolds given in [15] where they were called isospectral deformations. These are deformations of a classical Riemannian manifold and satisfy all the properties of noncommutative spin geometry [13]. They are the content of the following result taken from [15],

Theorem 3. Let $M$ be a compact spin Riemannian manifold whose isometry group has rank $r \geq 2$. Then $M$ admits a natural one parameter isospectral deformation to noncommutative geometries $M_{\theta}$.

The idea of the construction is to deform the standard spectral triple describing the Riemannian geometry of $M$ along a torus embedded in the isometry group, thus obtaining a family of spectral triples describing noncommutative geometries.

\subsection{Deforming a torus action}

Let $M$ be an $m$ dimensional compact Riemannian manifold equipped with an isometric smooth action $\sigma$ of an $n$-torus $\mathbb{T}^{n}, n \geq 2$. We denote by $\sigma$ also the corresponding action of $\mathbb{T}^{n}$ by automorphisms - obtained by pull-backs - on the algebra $C^{\infty}(M)$ of smooth functions on $M$.

The algebra $C^{\infty}(M)$ may be decomposed into spectral subspaces which are indexed by the dual group $\mathbb{Z}^{n}=\widehat{\mathbb{T}}^{n}$. Now, with $s=\left(s_{1}, \cdots, s_{n}\right) \in \mathbb{T}^{n}$, each $r \in \mathbb{Z}^{n}$ yields a character of $\mathbb{T}^{n}, e^{2 \pi i s} \mapsto e^{2 \pi i r \cdot s}$, with the scalar product $r \cdot s:=r_{1} s_{1}+\cdots+r_{n} s_{n}$. The $r$-th spectral subspace for the action $\sigma$ of $\mathbb{T}^{n}$ on $C^{\infty}(M)$ consists of those smooth functions $f_{r}$ for which

$$
\sigma_{s}\left(f_{r}\right)=e^{2 \pi i r \cdot s} f_{r},
$$

and each $f \in C^{\infty}(M)$ is the sum of a unique series $f=\sum_{r \in \mathbb{Z}^{n}} f_{r}$, which is rapidly convergent in the Fréchet topology of $C^{\infty}(M)$ (see [33] for more details). Let now $\theta=$ $\left(\theta_{j k}=-\theta_{k j}\right)$ be a real antisymmetric $n \times n$ matrix. The $\theta$-deformation of $C^{\infty}(M)$ may be defined by replacing the ordinary product by a deformed product, given on spectral subspaces by

$$
f_{r} \times_{\theta} g_{r^{\prime}}:=f_{r} \sigma_{\frac{1}{2} r \cdot \theta}\left(g_{r^{\prime}}\right)=e^{\pi i r \cdot \theta \cdot r^{\prime}} f_{r} g_{r^{\prime}}
$$


where $r \cdot \theta$ is the element in $\mathbb{R}^{n}$ with components $(r \cdot \theta)_{k}=\sum r_{j} \theta_{j k}$ for $k=1, \ldots, n$. The product in (3.2) is then extended linearly to all functions in $C^{\infty}(M)$. We denote the space $C^{\infty}(M)$ endowed with the product $\times_{\theta}$ by $C^{\infty}\left(M_{\theta}\right)$. The action $\sigma$ of $\mathbb{T}^{n}$ on $C^{\infty}(M)$ extends to an action on $C^{\infty}\left(M_{\theta}\right)$ given again by (3.1) on the homogeneous elements.

Next, let us take $M$ to be a spin manifold with $\mathcal{H}:=L^{2}(M, \mathcal{S})$ the Hilbert space of spinors and $D$ the usual Dirac operator of the metric of $M$. Smooth functions act on spinors by pointwise multiplication thus giving a representation $\pi: C^{\infty}(M) \rightarrow \mathcal{B}(\mathcal{H})$, the latter being the algebra of bounded operators on $\mathcal{H}$.

There is a double cover $c: \widetilde{\mathbb{T}}^{n} \rightarrow \mathbb{T}^{n}$ and a representation of $\widetilde{\mathbb{T}}^{n}$ on $\mathcal{H}$ by unitary operators $U(s), s \in \widetilde{\mathbb{T}}^{n}$, so that

$$
U(s) D U(s)^{-1}=D
$$

since the torus action is assumed to be isometric, and such that for all $f \in C^{\infty}(M)$,

$$
U(s) \pi(f) U(s)^{-1}=\pi\left(\sigma_{c(s)}(f)\right) .
$$

Recall that an element $T \in \mathcal{B}(\mathcal{H})$ is called smooth for the action of $\widetilde{\mathbb{T}}^{n}$ if the map

$$
\widetilde{\mathbb{T}}^{n} \ni s \mapsto \alpha_{s}(T):=U(s) T U(s)^{-1},
$$

is smooth for the norm topology. From its very definition, $\alpha_{s}$ coincides on $\pi\left(C^{\infty}(M)\right) \subset$ $\mathcal{B}(\mathcal{H})$ with the automorphism $\sigma_{c(s)}$. Much as it was done before for the smooth functions, we shall use the torus action to give a spectral decomposition of smooth elements of $\mathcal{B}(\mathcal{H})$. Any such a smooth element $T$ is written as a (rapidly convergent) series $T=\sum T_{r}$ with $r \in \mathbb{Z}^{n}$ and each $T_{r}$ is homogeneous of degree $r$ under the action of $\widetilde{\mathbb{T}}^{n}$, i.e.

$$
\alpha_{s}\left(T_{r}\right)=e^{2 \pi i r \cdot s} T_{r}, \quad \forall s \in \widetilde{\mathbb{T}}^{n} .
$$

Let $\left(P_{1}, P_{2}, \ldots, P_{n}\right)$ be the infinitesimal generators of the action of $\widetilde{\mathbb{T}}^{n}$ so that we can write $U(s)=\exp 2 \pi i s \cdot P$. Now, with $\theta$ a real $n \times n$ anti-symmetric matrix as above, one defines a twisted representation of the smooth elements of $\mathcal{B}(\mathcal{H})$ on $\mathcal{H}$ by

$$
L_{\theta}(T):=\sum_{r} T_{r} U\left(\frac{1}{2} r \cdot \theta\right)=\sum_{r} T_{r} \exp \left\{\pi i r_{j} \theta_{j k} P_{k}\right\}
$$

The twist $L_{\theta}$ commutes with the action $\alpha_{s}$ of $\widetilde{\mathbb{T}}^{n}$ and preserves the spectral components of smooth operators:

$$
\alpha_{s}\left(L_{\theta}\left(T_{r}\right)\right)=U(s) T U\left(\frac{1}{2} r \cdot \theta\right) U(s)^{-1}=U(s) T U(s)^{-1} U\left(\frac{1}{2} r \cdot \theta\right)=e^{2 \pi i r \cdot s} L_{\theta}\left(T_{r}\right) .
$$

Taking smooth functions on $M$ as elements of $\mathcal{B}(\mathcal{H})$, via the representation $\pi$, the previous definition gives an algebra $L_{\theta}\left(C^{\infty}(M)\right)$ which we may think of as a representation (as bounded operators on $\mathcal{H}$ ) of the algebra $C^{\infty}\left(M_{\theta}\right)$. Indeed, by the very definition of the product $x_{\theta}$ in (3.2) one establishes that

$$
L_{\theta}\left(f \times_{\theta} g\right)=L_{\theta}(f) L_{\theta}(g),
$$


proving that the algebra $C^{\infty}(M)$ equipped with the product $\times_{\theta}$ is isomorphic to the algebra $L_{\theta}\left(C^{\infty}(M)\right)$. It is shown in 33 that there is a natural completion of the algebra $C^{\infty}\left(M_{\theta}\right)$ to a $C^{*}$-algebra $C\left(M_{\theta}\right)$ whose smooth subalgebra - under the extended action of $\mathbb{T}^{n}$ - is precisely $C^{\infty}\left(M_{\theta}\right)$. Thus, we can understand $L_{\theta}$ as a quantization map from

$$
L_{\theta}: C^{\infty}(M) \rightarrow C^{\infty}\left(M_{\theta}\right)
$$

which provides a strict deformation quantization in the sense of Rieffel. More generally, in 33] one considers a (not necessarily commutative) $C^{*}$-algebra $A$ carrying an action of $\mathbb{R}^{n}$. For an anti-symmetric $n \times n$ matrix $\theta$, one defines a star product $\times_{\theta}$ between elements in $A$ much as we did before. The algebra $A$ equipped with the product $\times_{\theta}$ gives rise to a $C^{*}$-algebra denoted by $A_{\theta}$. Then the collection $\left\{A_{\hbar \theta}\right\}_{\hbar \in[0,1]}$ is a continuous family of $C^{*}$-algebras providing a strict deformation quantization in the direction of the Poisson structure on $A$ defined by the matrix $\theta$.

Our case of interest corresponds to the choice $A=C(M)$ with an action of $\mathbb{R}^{n}$ that is periodic or, in other words, an action of $\mathbb{T}^{n}$. The smooth elements in the deformed algebra make up the algebra $C^{\infty}\left(M_{\theta}\right)$. The quantization map will play a key role in what follows, allowing us to extend differential geometric techniques from $M$ to the noncommutative space $M_{\theta}$.

It was shown in [15] that the datum $\left(L_{\theta}\left(C^{\infty}(M)\right), \mathcal{H}, D\right)$ satisfies all properties of a noncommutative spin geometry [13] (see also [22]); there is also a grading $\gamma$ (for the even case) and a real structure $J$. In particular, boundedness of the commutators $\left[D, L_{\theta}(f)\right]$ for $f \in C^{\infty}(M)$ follows from $\left[D, L_{\theta}(f)\right]=L_{\theta}([D, f]), D$ being of degree 0 (since $\mathbb{T}^{n}$ acts by isometries, each $P_{k}$ commutes with $D$ ). This noncommutative geometry is an isospectral deformation of the classical Riemannian geometry of $M$, in that the spectrum of the operator $D$ coincides with that of the Dirac operator $D$ on $M$. Thus all spectral properties are unchanged. In particular, the triple is $m^{+}$-summable and there is a noncommutative integral as a Dixmier trace [17],

$$
f L_{\theta}(f):=\operatorname{Tr}_{\omega}\left(L_{\theta}(f)|D|^{-m}\right)
$$

with $f \in C^{\infty}\left(M_{\theta}\right)$ understood in its representation on $\mathcal{H}$. A drastic simplification of this noncommutative integral is given by the Lemma [20, Prop. 5.1].

Lemma 4. If $f \in C^{\infty}\left(M_{\theta}\right)$ then

$$
f L_{\theta}(f)=\int_{M} f \mathrm{~d} \nu
$$

Proof. Any element $f \in C^{\infty}\left(M_{\theta}\right)$ is given as an infinite sum of functions that are homogeneous under the action of $\mathbb{T}^{n}$. Let us therefore assume that $f$ is homogeneous of degree $k$ so that $\sigma_{s}\left(L_{\theta}(f)\right)=L_{\theta}\left(\sigma_{s}(f)\right)=e^{2 \pi i k \cdot s} L_{\theta}(f)$. From the tracial property of the noncommutative integral and the invariance of $D$ under the action of $\mathbb{T}^{n}$, we see that

$$
\operatorname{Tr}_{\omega}\left(\sigma_{s}\left(L_{\theta}(f)\right)|D|^{-m}\right)=\operatorname{Tr}_{\omega}\left(U(s) L_{\theta}(f) U(s)^{-1}|D|^{-m}\right)=\operatorname{Tr}_{\omega}\left(L_{\theta}(f)|D|^{-m}\right) .
$$

In other words, $e^{2 \pi i k \cdot s} \operatorname{Tr}_{\omega}\left(L_{\theta}(f)|D|^{-m}\right)=\operatorname{Tr}_{\omega}\left(L_{\theta}(f)|D|^{-m}\right)$ from which we infer that this trace vanishes if $k \neq 0$. If $k=0$, then $L_{\theta}(f)=f$, leading to the desired result. 


\subsection{The manifold $M_{\theta}$ as a fixed point algebra}

A different but equivalent approach to these noncommutative manifolds $M_{\theta}$ was introduced in [14]. In there the algebra $C^{\infty}\left(M_{\theta}\right)$ is identified as a fixed point subalgebra of $C^{\infty}(M) \otimes C^{\infty}\left(\mathbb{T}_{\theta}^{n}\right)$ where $C^{\infty}\left(\mathbb{T}_{\theta}^{n}\right)$ is the algebra of smooth functions on the noncommutative torus. This identification was shown to be useful in extending techniques from commutative differential geometry on $M$ to the noncommutative space $M_{\theta}$.

We recall the definition of the noncommutative $n$-torus $\mathbb{T}_{\theta}^{n}[32]$. Let $\theta=\left(\theta_{j k}\right)$ be a real $n \times n$ anti-symmetric matrix as before, and let $\lambda^{j k}=e^{2 \pi i \theta_{j k}}$. The unital $*$-algebra $\mathcal{A}\left(\mathbb{T}_{\theta}^{n}\right)$ of polynomial functions on $\mathbb{T}_{\theta}^{n}$ is generated by $n$ unitary elements $U^{k}, k=1, \ldots, n$, with relations

$$
U^{j} U^{k}=\lambda^{j k} U^{k} U^{j}, \quad j, k=1, \ldots, n .
$$

The polynomial algebra is extended to the universal $C^{*}$-algebra with the same generators. There is a natural action of $\mathbb{T}^{n}$ on $\mathcal{A}\left(\mathbb{T}_{\theta}^{n}\right)$ by $*$-automorphisms given by $\tau_{s}\left(U^{k}\right)=e^{2 \pi i s_{k}} U^{k}$ with $s=\left(s_{k}\right) \in \mathbb{T}^{n}$. The corresponding infinitesimal generators $X_{k}$ of the action are algebra derivations given explicitly on the generators by $X_{k}\left(U^{j}\right)=2 \pi i \delta_{k}^{j}$. They are used [10] to construct the pre- $C^{*}$-algebra $C^{\infty}\left(\mathbb{T}_{\theta}^{n}\right)$ of smooth functions on $\mathbb{T}_{\theta}^{n}$, which is the completion of $\mathcal{A}\left(\mathbb{T}_{\theta}^{n}\right)$ with respect to the locally convex topology generated by the seminorms,

$$
|u|_{r}:=\sup _{r_{1}+\cdots+r_{n} \leq r}\left\|X_{1}^{r_{1}} \cdots X_{n}^{r_{n}}(u)\right\|
$$

and $\|\cdot\|$ is the $C^{*}$-norm. The algebra $C^{\infty}\left(\mathbb{T}_{\theta}^{n}\right)$ turns out to be a nuclear Fréchet space and one can unambiguously take the completed tensor product $C^{\infty}(M) \bar{\otimes} C^{\infty}\left(\mathbb{T}_{\theta}^{n}\right)$. Then, one defines $\left(C^{\infty}(M) \bar{\otimes} C^{\infty}\left(\mathbb{T}_{\theta}^{n}\right)\right)^{\sigma \otimes \tau^{-1}}$ as the fixed point subalgebra of $C^{\infty}(M) \bar{\otimes} C^{\infty}\left(\mathbb{T}_{\theta}^{n}\right)$ consisting of elements $a$ in the tensor product that are invariant under the diagonal action of $\mathbb{T}^{n}$, i.e. such that $\sigma_{s} \otimes \tau_{-s}(a)=a$ for all $s \in \mathbb{T}^{n}$. The noncommutative manifold $M_{\theta}$ is defined by "duality" by setting

$$
C^{\infty}\left(M_{\theta}\right):=\left(C^{\infty}(M) \bar{\otimes} C^{\infty}\left(\mathbb{T}_{\theta}^{n}\right)\right)^{\sigma \otimes \tau^{-1}} .
$$

As the notation suggests, the algebra $C^{\infty}\left(M_{\theta}\right)$ turns out to be isomorphic to the algebra $L_{\theta}\left(C^{\infty}(M)\right)$ defined in the previous section.

Next, let $\mathcal{S}$ be a spin bundle over $M$ and $D$ the Dirac operator on $\Gamma^{\infty}(M, \mathcal{S})$, the $C^{\infty}(M)$-module of smooth sections of $\mathcal{S}$. The action of the group $\mathbb{T}^{n}$ on $M$ does not lift directly to the spinor bundle. Rather, there is a double cover $c: \widetilde{\mathbb{T}}^{n} \rightarrow \mathbb{T}^{n}$ and a group homomorphism $\widetilde{s} \rightarrow V_{\widetilde{s}}$ of $\widetilde{\mathbb{T}}^{n}$ into $\operatorname{Aut}(\mathcal{S})$ covering the action of $\mathbb{T}^{n}$ on $M$,

$$
V_{\widetilde{s}}(f \psi)=\sigma_{c(s)}(f) V_{\widetilde{s}}(\psi),
$$

for $f \in C^{\infty}(M)$ and $\psi \in \Gamma^{\infty}(M, \mathcal{S})$. According to [14, the proper notion of smooth sections $\Gamma^{\infty}\left(M_{\theta}, \mathcal{S}\right)$ of a spinor bundle on $M_{\theta}$ are elements of $\Gamma^{\infty}(M, \mathcal{S}) \widehat{\otimes} C^{\infty}\left(\mathbb{T}_{\theta / 2}^{n}\right)$ which are invariant under the diagonal action $V \times \widetilde{\tau}^{-1}$ of $\widetilde{\mathbb{T}}^{n}$. Here $\widetilde{s} \mapsto \widetilde{\tau}_{\widetilde{s}}$ is the canonical action of $\widetilde{\mathbb{T}}^{n}$ on $\mathcal{A}\left(\mathbb{T}_{\theta / 2}^{n}\right)$. Since the Dirac operator $D$ commutes with $V_{\widetilde{s}}$ (remember that the torus action is isometric) one can restrict $D \otimes$ id to the fixed point elements $\Gamma^{\infty}\left(M_{\theta}, \mathcal{S}\right)$.

Then, let $L^{2}(M, \mathcal{S})$ be the space of square integrable spinors on $M$ and let $L^{2}\left(\mathbb{T}_{\theta / 2}^{n}\right)$ be the completion of $C^{\infty}\left(\mathbb{T}_{\theta / 2}^{n}\right)$ in the norm $a \mapsto\|a\|=\operatorname{tr}\left(a^{*} a\right)^{1 / 2}$, with tr the usual trace 
on $C^{\infty}\left(\mathbb{T}_{\theta / 2}^{n}\right)$. The diagonal action $V \times \widetilde{\tau}^{-1}$ of $\widetilde{\mathbb{T}}^{n}$ extends to $L^{2}(M, \mathcal{S}) \otimes L^{2}\left(\mathbb{T}_{\theta / 2}^{n}\right)$ (where it becomes $U \times \tau)$ and one defines $L^{2}\left(M_{\theta}, \mathcal{S}\right)$ to be the fixed point Hilbert subspace. If $D$ also denotes the closure of the Dirac operator on $L^{2}(M, \mathcal{S})$, one still denotes by $D$ the operator $D \otimes$ id on $L^{2}(M, \mathcal{S}) \otimes L^{2}\left(\mathbb{T}_{\theta / 2}^{n}\right)$ when restricted to $L^{2}\left(M_{\theta}, \mathcal{S}\right)$. The triple $\left(C^{\infty}\left(M_{\theta}\right), L^{2}\left(M_{\theta}, \mathcal{S}\right), D\right)$ is an $m^{+}$-summable noncommutative spin geometry.

\subsection{Vector bundles on $M_{\theta}$}

Noncommutative vector bundles on $M_{\theta}$, i.e. finite projective modules over $C^{\infty}\left(M_{\theta}\right)$, were obtained in [14] as fixed point submodules of $\Gamma^{\infty}(M, E) \otimes C^{\infty}\left(\mathbb{T}_{\theta}^{n}\right)$ under some diagonal action of the torus $\mathbb{T}^{n}$. Here $\Gamma^{\infty}(M, E)$ denotes the $C^{\infty}(M)$-bimodule of smooth sections of a vector bundle $E \rightarrow M$. We will presently give an equivalent description of these modules over $C^{\infty}\left(M_{\theta}\right)$ in terms of a kind of $*$-product.

Let $E$ be a $\sigma$-equivariant vector bundle over $M$, that is a bundle which carries an action $V$ of $\mathbb{T}^{n}$ by automorphisms, covering the action $\sigma$ of $\mathbb{T}^{n}$ on $M$,

$$
V_{s}(f \psi)=\sigma_{s}(f) V_{s}(\psi), \quad \forall f \in C^{\infty}(M), \psi \in \Gamma^{\infty}(M, E) .
$$

We also assume that the topology on the Fréchet $C^{\infty}(M)$-bimodule $\Gamma^{\infty}(M, E)$ is given in terms of a family of $\mathbb{T}^{n}$-invariant seminorms $\|\cdot\|_{i}$.

We define the $C^{\infty}\left(M_{\theta}\right)$-bimodule $\Gamma^{\infty}\left(M_{\theta}, E\right)$ as the vector space $\Gamma^{\infty}(M, E)$ but with deformed bimodule structure given by

$$
\begin{aligned}
& f \triangleright_{\theta} \psi=\sum_{k} f_{k} V_{\frac{1}{2} k \cdot \theta}(\psi), \\
& \psi \triangleleft_{\theta} f=\sum_{k} V_{-\frac{1}{2} k \cdot \theta}(\psi) f_{k} ;
\end{aligned}
$$

here $f=\sum_{k} f_{k}$ with $f_{k} \in C^{\infty}(M)$ homogeneous of degree $k$ under the action of $\mathbb{T}^{n}$ - as in (3.5) - and $\psi$ is a smooth section of $E$. By using the explicit expression (3.2) for the star product and equation (3.14), one checks that these are indeed actions of $C^{\infty}\left(M_{\theta}\right)$. Moreover, the invariance of $\|\cdot\|_{i}$ under the action of $\mathbb{T}^{n}$ makes both actions continuous, turning $\Gamma^{\infty}\left(M_{\theta}, E\right)$ into a Fréchet $C^{\infty}\left(M_{\theta}\right)$-bimodule.

The $C^{\infty}\left(M_{\theta}\right)$-bimodule $\Gamma^{\infty}\left(M_{\theta}, E\right)$ is finite projective [14] and still carries an action $V$ of $\mathbb{T}^{n}$ with equivariance as in (3.14) for both the left and right action of $C^{\infty}\left(M_{\theta}\right)$ : the group $\mathbb{T}^{n}$ being abelian, one directly establishes that

$$
V_{s}\left(f \triangleright_{\theta} \psi\right)=\sigma_{s}(f) \triangleright_{\theta} V_{s}(\psi), \quad \forall f \in C^{\infty}\left(M_{\theta}\right), \psi \in \Gamma^{\infty}\left(M_{\theta}, E\right),
$$

and a similar property for the right structure $\triangleleft_{\theta}$. In fact, since the category of $\sigma$ equivariant finite projective module over $C^{\infty}\left(M_{\theta}\right)$ is equivalent to the category of finite projectve modules over $C^{\infty}\left(M_{\theta}\right) \rtimes_{\sigma} \mathbb{T}^{n}$ (see [23]) for all $\theta$ (in particular $\theta=0$ ), the isomorphism $C^{\infty}\left(M_{\theta}\right) \rtimes_{\sigma} \mathbb{T}^{n} \simeq C^{\infty}(M) \rtimes_{\sigma} \mathbb{T}^{n}$ shows that all $\sigma$-equivariant finite projective modules over $C^{\infty}\left(M_{\theta}\right)$ are of the above type [14, Proof of Prop. 5]. This also reflects the result in 34] that the K-groups of a $C^{*}$-algebra deformed by an action of $\mathbb{R}^{n}$ are isomorphic to the $\mathrm{K}$-groups of the original $C^{*}$-algebra: as mentioned above, the noncommutative manifolds $M_{\theta}$ are a special case - in which the starting algebra is commutative 
and the action periodic - of the general formulation in [33] of deformations of $C^{*}$-algebras under an action of $\mathbb{R}^{n}$.

Although we defined the above left and right actions on sections with respect to an action of $\mathbb{T}^{n}$ on the vector bundle $E$, the same construction can be done for vector bundles carrying an action of the double cover $\widetilde{\mathbb{T}}^{n}$. We have already seen an example of this double cover action for the spinor bundle, for which we defined a left action of $C^{\infty}\left(M_{\theta}\right)$ using the twisted representation (3.6).

From the very definition of $\Gamma^{\infty}\left(M_{\theta}, E\right)$ the following lemma is true.

Lemma 5. If $E \simeq F$ as $\sigma$-equivariant vector bundles, then $\Gamma^{\infty}\left(M_{\theta}, E\right) \simeq \Gamma^{\infty}\left(M_{\theta}, F\right)$ as $C^{\infty}\left(M_{\theta}\right)$-bimodules.

\subsection{Differential calculus on $M_{\theta}$}

It is straightforward to construct a differential calculus on $M_{\theta}$. This can be done in two equivalent manners, either by extending to forms the quantization maps, or by using the general construction in [12] by means of the Dirac operator.

Firstly, let $(\Omega(M), \mathrm{d})$ be the usual differential calculus on $M$, with $\mathrm{d}$ the exterior derivative. This becomes a Fréchet algebra if we consider $\Omega(M)$ as the space of smooth sections of a bundle over $M$. Moreover, it carries an action $\sigma$ of $\mathbb{T}^{n}$ by automorphisms which commutes with the differential d. Again, we assume that the seminorms defining the Fréchet topology of $\Omega(M)$ are $\mathbb{T}^{n}$-invariant.

The quantization map $L_{\theta}: C^{\infty}(M) \rightarrow C^{\infty}\left(M_{\theta}\right)$ is extended to $\Omega(M)$ by imposing that it commutes with d. The image $L_{\theta}(\Omega(M))$ will be denoted $\Omega\left(M_{\theta}\right)$ and becomes a Fréchet algebra with the induced seminorms from $\Omega(M)$. Equivalently, $\Omega\left(M_{\theta}\right)$ could be defined to be $\Omega(M)$ as a vector space but equipped with an "exterior star product" which is the extension of the product (3.2) to $\Omega(M)$ by the requirement that it commutes with $\mathrm{d}$. Indeed, since the action of $\mathbb{T}^{n}$ commutes with $\mathrm{d}$, an element in $\Omega(M)$ can be decomposed into a sum of a rapidly convergent series of homogeneous elements for the action of $\mathbb{T}^{n}$ as was done for $C^{\infty}(M)$. Then one defines a star product $\times_{\theta}$ on homogeneous elements in $\Omega(M)$ as in (3.2) and denotes $\Omega\left(M_{\theta}\right)=\left(\Omega(M), \times_{\theta}\right)$. This construction is in concordance with the previous section, when $\Omega(M)$ is considered as a $C^{\infty}(M)$-bimodule of sections. The extended action of $\mathbb{T}^{n}$ from $C^{\infty}(M)$ to $\Omega(M)$ is used to endow the space $\Omega\left(M_{\theta}\right)$ with the structure of a $C^{\infty}\left(M_{\theta}\right)$-bimodule with a left and right action given in (3.15)-(3.16) .

As mentioned, a differential calculus $\Omega_{D}\left(C^{\infty}\left(M_{\theta}\right)\right)$ on $C^{\infty}\left(M_{\theta}\right)$ can also be obtained from the general procedure [12] using the isospectral Dirac operator $D$ on $M_{\theta}$ defined above. The $C^{\infty}\left(M_{\theta}\right)$-bimodule $\Omega_{D}^{p}\left(C^{\infty}\left(M_{\theta}\right)\right)$ of $p$-forms is made of classes of operators

$$
\omega=\sum_{j} a_{0}^{j}\left[D, a_{1}^{j}\right] \cdots\left[D, a_{p}^{j}\right], \quad a_{i}^{j} \in C^{\infty}\left(M_{\theta}\right)
$$

modulo the sub-bimodule of operators

$$
\left\{\sum_{j}\left[D, b_{0}^{j}\right]\left[D, b_{1}^{j}\right] \cdots\left[D, b_{p-1}^{j}\right]: b_{i}^{j} \in C^{\infty}\left(M_{\theta}\right), b_{0}^{j}\left[D, b_{1}^{j}\right] \cdots\left[D, b_{p-1}^{j}\right]=0\right\} .
$$


The exterior differential $\mathrm{d}_{D}$ is given by

$$
\mathrm{d}_{D}\left[\sum_{j} a_{0}^{j}\left[D, a_{1}^{j}\right] \cdots\left[D, a_{p}^{j}\right]\right]=\left[\sum_{j}\left[D, a_{0}^{j}\right]\left[D, a_{1}^{j}\right] \cdots\left[D, a_{p}^{j}\right]\right],
$$

and satisfies $\mathrm{d}_{D}^{2}=0$. One also introduces an inner product on forms by declaring that forms of different degree are orthogonal, while for two $p$-forms $\omega_{1}, \omega_{2}$, the product is

$$
\left(\omega_{1}, \omega_{2}\right)_{D}=f \omega_{1}^{*} \omega_{2}
$$

Here the noncommutative integral is the natural extension of the one in (3.10),

$$
f T:=\operatorname{Tr}_{\omega}\left(T|D|^{-m}\right)
$$

with $T$ an element in a suitable class of operators. Not surprisingly, these two constructions of forms agree [14], that is, the differential calculi $\Omega\left(M_{\theta}\right)$ and $\Omega_{D}\left(C^{\infty}\left(M_{\theta}\right)\right)$ are isomorphic. This allows us in particular to integrate forms of top dimension, by defining

$$
\int_{M_{\theta}} \omega:=f \omega_{D}, \quad \omega \in \Omega^{m}\left(M_{\theta}\right)
$$

where $\omega_{D}$ denotes the element in $\Omega_{D}^{m}\left(C^{\infty}\left(M_{\theta}\right)\right.$ ) corresponding to $\omega$ (replacing every $\mathrm{d}$ in $\omega$ by $\left.\mathrm{d}_{D}\right)$. We have the following noncommutative Stokes theorem.

Lemma 6. If $\omega \in \Omega^{m-1}\left(M_{\theta}\right)$ then

$$
\int_{M_{\theta}} \mathrm{d} \omega=0
$$

Proof. From the definition of the noncommutative integral,

$$
\int_{M_{\theta}} \mathrm{d} \omega=f \mathrm{~d}_{D} \omega_{D}=f \mathrm{~d}_{D} L_{\theta}\left(\omega_{D}^{(0)}\right)
$$

with $\omega_{D}^{(0)}$ the classical counterpart of $\omega$, i.e. $\omega=L_{\theta}\left(\omega_{D}^{(0)}\right)$. At this point one remembers that $D$ commutes with $L_{\theta}$ (see Section 3.1), and realizes that there is an analogue of Lemma 4 for forms, i.e. $f L_{\theta}(T)=\int_{M} T$. One concludes that the above integral vanishes since it vanishes in the classical case.

The next ingredient is a Hodge star operator on $\Omega\left(M_{\theta}\right)$. Classically, the Hodge star operator is a map $*: \Omega^{p}(M) \rightarrow \Omega^{m-p}(M)$ depending only on the conformal class of the metric on $M$. On the one end, since $\mathbb{T}^{n}$ acts by isometries, it leaves the conformal structure invariant and therefore, it commutes with $*$. On the other hand, with the isospectral deformation one does not change the metric. Thus it makes sense to define a map $*_{\theta}: \Omega^{p}\left(M_{\theta}\right) \rightarrow \Omega^{m-p}\left(M_{\theta}\right)$ by

$$
*_{\theta} L_{\theta}(\omega)=L_{\theta}(* \omega), \quad \text { for } \quad \omega \in \Omega(M) .
$$


With this Hodge operator, there is an alternative definition of an inner product on $\Omega\left(M_{\theta}\right)$. Given that $*_{\theta}$ maps $\Omega^{p}\left(M_{\theta}\right)$ to $\Omega^{m-p}\left(M_{\theta}\right)$, we can define for $\alpha, \beta \in \Omega^{p}\left(M_{\theta}\right)$

$$
(\alpha, \beta)_{2}=f *_{\theta}\left(\alpha^{*} *_{\theta} \beta\right)
$$

since $*_{\theta}\left(\alpha^{*} *_{\theta} \beta\right)$ is an element in $C^{\infty}\left(M_{\theta}\right)$.

Lemma 7. Under the isomorphism $\Omega_{D}\left(C^{\infty}\left(M_{\theta}\right)\right) \simeq \Omega\left(M_{\theta}\right)$, the inner product $(\cdot, \cdot)_{2}$ coincides with $(\cdot, \cdot)_{D}$.

Proof. Let $\omega_{1}, \omega_{2}$ be two forms in $\Omega_{D}\left(C^{\infty}(M)\right)$, so that $L_{\theta}\left(\omega_{i}\right)$ are two generic forms in $\Omega_{D}\left(C^{\infty}\left(M_{\theta}\right)\right) \simeq L_{\theta}(\Omega(M))=\Omega\left(M_{\theta}\right)$. Then, using Lemma 4 it follows that

$$
f L_{\theta}\left(\omega_{1}\right)^{*} L_{\theta}\left(\omega_{2}\right)=f L_{\theta}\left(\omega_{1}^{*} \times_{\theta} \omega_{2}\right)=f \omega_{1}^{*} \times_{\theta} \omega_{2}
$$

Now, the inner product $(,)_{D}$ coincides with $(,)_{2}$ as defined by (3.25) in the classical case - under the above isomorphism $\Omega_{D}\left(C^{\infty}(M)\right) \simeq \Omega(M)$; see for example [12, VI.1]. It follows that the above expression equals

$$
f *\left(\omega_{1}^{*} \times_{\theta}\left(* \omega_{2}\right)\right)=f *_{\theta}\left(L_{\theta}\left(\omega_{1}\right)^{*}\left(*_{\theta} L_{\theta}\left(\omega_{2}\right)\right)\right),
$$

using Lemma 4 for forms once more, together with the defining property of $*_{\theta}$.

Lemma 8. The formal adjoint $\mathrm{d}^{*}$ of $\mathrm{d}$ with respect to the inner product $(\cdot, \cdot)_{2}-i$.e. so that $\left(\mathrm{d}^{*} \alpha, \beta\right)_{2}=(\alpha, \mathrm{d} \beta)_{2}-$ is given on $\Omega^{p}\left(M_{\theta}\right)$ by

$$
\mathrm{d}^{*}=(-1)^{m(p+1)+1} *_{\theta} \mathrm{d} *_{\theta} .
$$

Proof. Just as in the classical case, this follows from Stokes Lemma 6, together with the observation that

$$
\int_{M_{\theta}} \omega=f *_{\theta} \omega, \quad \omega \in \Omega^{m}\left(M_{\theta}\right),
$$

again established from the classical case by means of the mentioned analogue of Lemma 4 for forms.

Remark 9. The algebra $\Omega\left(M_{\theta}\right)$ can also be defined as a fixed point algebra [14]. The action $\sigma$ of $\mathbb{T}^{n}$ on $\Omega(M)$ allows one to define $\Omega\left(M_{\theta}\right)$ by $\left(\Omega(M) \bar{\otimes} C^{\infty}\left(\mathbb{T}_{\theta}^{n}\right)\right)^{\sigma \otimes \tau^{-1}}$. Furthermore, since the exterior derivative $\mathrm{d}$ on $\Omega(M)$ commutes with the action of $\mathbb{T}^{n}$, the differential $\mathrm{d}_{\theta}$, for the fixed point algebra is defined as $\mathrm{d}_{\theta}=\mathrm{d} \otimes \mathrm{id}$. Similarly, the Hodge star operator takes the form $*_{\theta}=* \otimes$ id with $*$ the classical Hodge operator.

\section{Gauge theory on the sphere $S_{\theta}^{4}$}

We now apply the general scheme of noncommutative gauge field theories - as developed in Section 2 - to the case of the $\mathrm{SU}(2)$ noncommutative principal bundle $S_{\theta^{\prime}}^{7} \rightarrow S_{\theta}^{4}$ constructed in [27]. This will also make more explicit all the constructions above. It is worth stressing that what follows is valid for more general $\theta$-deformed $G$-principal bundle. We will come back to this point later in the paper. 


\subsection{The principal fibration $S_{\theta^{\prime}}^{7} \rightarrow S_{\theta}^{4}$}

The $\mathrm{SU}(2)$ noncommutative principal fibration $S_{\theta^{\prime}}^{7} \rightarrow S_{\theta}^{4}$ is given by an algebra inclusion $\mathcal{A}\left(S_{\theta}^{4}\right) \hookrightarrow \mathcal{A}\left(S_{\theta^{\prime}}^{7}\right)$. The algebra $\mathcal{A}\left(S_{\theta}^{4}\right)$ of polynomial functions on the sphere $S_{\theta}^{4}$ is generated by elements $z_{0}=z_{0}^{*}, z_{j}, z_{j}^{*}, j=1,2$, subject to relations

$$
z_{\mu} z_{\nu}=\lambda_{\mu \nu} z_{\nu} z_{\mu}, \quad z_{\mu} z_{\nu}^{*}=\lambda_{\nu \mu} z_{\nu}^{*} z_{\mu}, \quad z_{\mu}^{*} z_{\nu}^{*}=\lambda_{\mu \nu} z_{\nu}^{*} z_{\mu}^{*}, \quad \mu, \nu=0,1,2
$$

together with the spherical relation $\sum_{\mu} z_{\mu}^{*} z_{\mu}=1$. Here $\theta$ is a real parameter and the deformation parameters are given by $\lambda_{\mu \mu}=1$ and

$$
\lambda_{12}=\bar{\lambda}_{21}=: \lambda=e^{2 \pi i \theta}, \quad \lambda_{j 0}=\lambda_{0 j}=1, \quad j=1,2 .
$$

For $\theta=0$ one recovers the $*$-algebra of complex polynomial functions on the usual $S^{4}$.

The differential calculus $\Omega\left(S_{\theta}^{4}\right)$ is generated as a graded differential *-algebra by the elements $z_{\mu}, z_{\mu}^{*}$ in degree 0 and elements $d z_{\mu}, d z_{\mu}^{*}$ in degree 1 satisfying the relations,

$$
\begin{array}{ll}
d z_{\mu} d z_{\nu}+\lambda_{\mu \nu} d z_{\nu} d z_{\mu}=0, & d z_{\mu} d z_{\nu}^{*}+\lambda_{\nu \mu} d z_{\nu}^{*} d z_{\mu}=0 \\
z_{\mu} d z_{\nu}=\lambda_{\mu \nu} d z_{\nu} z_{\mu}, & z_{\mu} d z_{\nu}^{*}=\lambda_{\nu \mu} d z_{\nu}^{*} z_{\mu},
\end{array}
$$

with $\lambda_{\mu \nu}$ as before. There is a unique differential $\mathrm{d}$ on $\Omega\left(S_{\theta}^{4}\right)$ such that $\mathrm{d}: z_{\mu} \mapsto d z_{\mu}$ and the involution on $\Omega\left(S_{\theta}^{4}\right)$ is the graded extension of $z_{\mu} \mapsto z_{\mu}^{*}$ : $(\mathrm{d} \omega)^{*}=\mathrm{d} \omega^{*}$ and $\left(\omega_{1} \omega_{2}\right)^{*}=(-1)^{d_{1} d_{2}} \omega_{2}^{*} \omega_{1}^{*}$ for $\omega_{j}$ a form of degree $d_{j}$.

With $\lambda_{a b}^{\prime}=e^{2 \pi i \theta_{a b}^{\prime}}$ and $\left(\theta_{a b}^{\prime}\right)$ a real antisymmetric matrix, the algebra $\mathcal{A}\left(S_{\theta^{\prime}}^{7}\right)$ of polynomial functions on the sphere $S_{\theta^{\prime}}^{7}$ is generated by elements $\psi_{a}, \psi_{a}^{*}, a=1, \ldots, 4$, subject to relations

$$
\psi_{a} \psi_{b}=\lambda_{a b}^{\prime} \psi_{b} \psi_{a}, \quad \psi_{a} \psi_{b}^{*}=\lambda_{b a}^{\prime} \psi_{b}^{*} \psi_{a}, \quad \psi_{a}^{*} \psi_{b}^{*}=\lambda_{a b}^{\prime} \psi_{b}^{*} \psi_{a}^{*}
$$

and with the spherical relation $\sum_{a} \psi_{a}^{*} \psi_{a}=1$. Clearly, $\mathcal{A}\left(S_{\theta^{\prime}}^{7}\right)$ is a deformation of the *-algebra of complex polynomial functions on the sphere $S^{7}$. As before, a differential calculus $\Omega\left(S_{\theta^{\prime}}^{7}\right)$ can be defined to be generated by the elements $\psi_{a}, \psi_{a}^{*}$ in degree 0 and elements $d \psi_{a}, d \psi_{a}^{*}$ in degree 1 satisfying relations similar to the ones in (4.3).

In order to construct the noncommutative fibration over the given 4-sphere $S_{\theta}^{4}$ we need to select a particular noncommutative 7 dimensional sphere $S_{\theta^{\prime}}^{7}$. We take the one corresponding to the following deformation parameters

$$
\lambda_{a b}^{\prime}=\left(\begin{array}{cccc}
1 & 1 & \bar{\mu} & \mu \\
1 & 1 & \mu & \bar{\mu} \\
\mu & \bar{\mu} & 1 & 1 \\
\bar{\mu} & \mu & 1 & 1
\end{array}\right), \quad \mu=\sqrt{\lambda}, \quad \text { or } \quad \theta_{a b}^{\prime}=\frac{\theta}{2}\left(\begin{array}{cccc}
0 & 0 & -1 & 1 \\
0 & 0 & 1 & -1 \\
1 & -1 & 0 & 0 \\
-1 & 1 & 0 & 0
\end{array}\right) .
$$

The previous choice is essentially the only one 1 that allows the algebra $\mathcal{A}\left(S_{\theta^{\prime}}^{7}\right)$ to carry an action of the group $\mathrm{SU}(2)$ by automorphisms and such that the invariant subalgebra

\footnotetext{
${ }^{1}$ Compatibility requires that $\mu^{2}=\lambda$; we drop the case $\mu=-\sqrt{\lambda}$ since its "classical" limit would correspond to "anti-commuting" coordinates.
} 
coincides with $\mathcal{A}\left(S_{\theta}^{4}\right)$. The best way to see this is by means of the matrix-valued function on $\mathcal{A}\left(S_{\theta^{\prime}}^{7}\right)$ given by

$$
\Psi=\left(\begin{array}{cc}
\psi_{1} & -\psi_{2}^{*} \\
\psi_{2} & \psi_{1}^{*} \\
\psi_{3} & -\psi_{4}^{*} \\
\psi_{4} & \psi_{3}^{*}
\end{array}\right)
$$

Then the commutation relation of the algebra $\mathcal{A}\left(S_{\theta^{\prime}}^{7}\right)$ gives $\Psi^{\dagger} \Psi=\mathbb{I}_{2}$ and $p=\Psi \Psi^{\dagger}$ is a projection, $p^{2}=p=p^{\dagger}$, with entries in $\mathcal{A}\left(S_{\theta}^{4}\right)$. Indeed, the right action of $\mathrm{SU}(2)$ on $\mathcal{A}\left(S_{\theta^{\prime}}^{7}\right)$ is simply given by

$$
\alpha_{w}(\Psi)=\Psi w, \quad w=\left(\begin{array}{cc}
w_{1} & -\bar{w}_{2} \\
w_{2} & \bar{w}_{1}
\end{array}\right) \in \mathrm{SU}(2),
$$

from which the invariance under the $S U(2)$-action of the entries of $p$ follows at once. Explicitly,

$$
p=\frac{1}{2}\left(\begin{array}{cccc}
1+z_{0} & 0 & z_{1} & -\bar{\mu} z_{2}^{*} \\
0 & 1+z_{0} & z_{2} & \mu z_{1}^{*} \\
z_{1}^{*} & z_{2}^{*} & 1-z_{0} & 0 \\
-\mu z_{2} & \bar{\mu} z_{1} & 0 & 1-z_{0}
\end{array}\right),
$$

with the generators of $\mathcal{A}\left(S_{\theta}^{4}\right)$ given by

$$
\begin{aligned}
z_{0} & =\psi_{1}^{*} \psi_{1}+\psi_{2}^{*} \psi_{2}-\psi_{3}^{*} \psi_{3}-\psi_{4}^{*} \psi_{4} \\
& =2\left(\psi_{1}^{*} \psi_{1}+\psi_{2}^{*} \psi_{2}\right)-1=1-2\left(\psi_{3}^{*} \psi_{3}+\psi_{4}^{*} \psi_{4}\right), \\
z_{1} & =2\left(\mu \psi_{3}^{*} \psi_{1}+\psi_{2}^{*} \psi_{4}\right)=2\left(\psi_{1} \psi_{3}^{*}+\psi_{2}^{*} \psi_{4}\right) \\
z_{2} & =2\left(-\psi_{1}^{*} \psi_{4}+\bar{\mu} \psi_{3}^{*} \psi_{2}\right)=2\left(-\psi_{1}^{*} \psi_{4}+\psi_{2} \psi_{3}^{*}\right) .
\end{aligned}
$$

One straightforwardly computes that $z_{1}^{*} z_{1}+z_{1}^{*} z_{1}+z_{0}^{2}=1$ and the commutation rules $z_{1} z_{2}=\lambda z_{2} z_{1}, z_{1} z_{2}^{*}=\bar{\lambda} z_{2}^{*} z_{1}$, and that $z_{0}$ is central.

The relations (4.9) can also be expressed in the form,

$$
z_{\mu}=\sum_{a b} \psi_{a}^{*}\left(\gamma_{\mu}\right)_{a b} \psi_{b}, \quad z_{\mu}^{*}=\sum_{a b} \psi_{a}^{*}\left(\gamma_{\mu}^{*}\right)_{a b} \psi_{b}
$$

with $\gamma_{\mu}$ twisted $4 \times 4$ Dirac matrices given by

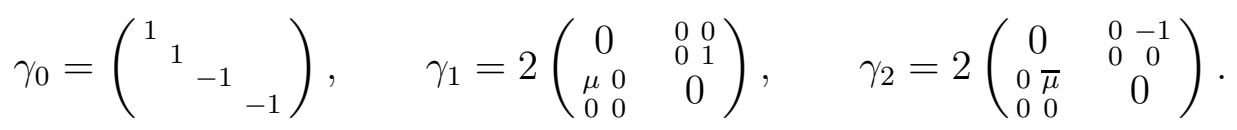

Note that as usual $\gamma_{0}$ is the grading

$$
\gamma_{0}=-\frac{1}{4}\left[\gamma_{1}, \gamma_{1}^{*}\right]\left[\gamma_{2}, \gamma_{2}^{*}\right]
$$

These matrices satisfy twisted Clifford algebra relations [14],

$$
\gamma_{j} \gamma_{k}+\lambda_{j k} \gamma_{k} \gamma_{j}=0, \quad \gamma_{j} \gamma_{k}^{*}+\lambda_{k j} \gamma_{k}^{*} \gamma_{j}=4 \delta_{j k} ; \quad(j, k=1,2) .
$$

There are compatible toric actions on $S_{\theta}^{4}$ and $S_{\theta^{\prime}}^{7}$. The torus $\mathbb{T}^{2}$ acts on $\mathcal{A}\left(S_{\theta}^{4}\right)$ as,

$$
\sigma_{s}\left(z_{0}, z_{1}, z_{2}\right)=\left(z_{0}, e^{2 \pi i s_{1}} z_{1}, e^{2 \pi i s_{2}} z_{2}\right), \quad s \in \mathbb{T}^{2} .
$$


This action is lifted to a double cover action on $\mathcal{A}\left(S_{\theta^{\prime}}^{7}\right)$. The double cover map $p: \widetilde{\mathbb{T}}^{2} \rightarrow \mathbb{T}^{2}$ is given explicitly by $p:\left(s_{1}, s_{2}\right) \mapsto\left(s_{1}+s_{2},-s_{1}+s_{2}\right)$. Then $\widetilde{\mathbb{T}}^{2}$ acts on the $\psi_{a}$ 's as,

$$
\widetilde{\sigma}:\left(\psi_{1}, \psi_{2}, \psi_{3}, \psi_{4}\right) \mapsto\left(e^{2 \pi i s_{1}} \psi_{1}, e^{-2 \pi i s_{1}} \psi_{2}, e^{-2 \pi i s_{2}} \psi_{3}, e^{2 \pi i s_{2}} \psi_{4}\right)
$$

Equation (4.9) shows that $\widetilde{\sigma}$ is indeed a lifting to $S_{\theta^{\prime}}^{7}$ of the action of $\mathbb{T}^{2}$ on $S_{\theta}^{4}$. Clearly, this compatibility is built in the construction of the Hopf fibration $S_{\theta^{\prime}}^{7} \rightarrow S_{\theta}^{4}$ as a deformation of the classical Hopf fibration $S^{7} \rightarrow S^{4}$ with respect to an action of $\mathbb{T}^{2}$, a fact that also dictated the form of the deformation parameter $\lambda^{\prime}$ in (4.5). As we shall see, the previous double cover of tori comes from a spin cover $\operatorname{Spin}_{\theta}(5)$ of $\mathrm{SO}_{\theta}(5)$ deforming the usual action of $\operatorname{Spin}(5)$ on $S^{7}$ as a double cover of the action of $\mathrm{SO}(5)$ on $S^{4}$.

In which sense the algebra inclusion $\mathcal{A}\left(S_{\theta}^{4}\right) \hookrightarrow \mathcal{A}\left(S_{\theta^{\prime}}^{7}\right)$ is a noncommutative principal bundle was explained in [27] to which we refer for more details. Presently we shall recall the construction of associated bundles.

\subsection{Associated bundles}

We shall work in the context of smooth functions on $S_{\theta^{\prime}}^{7}$ and $S_{\theta}^{4}$ as defined in general in Section 3. Let $\rho$ be any finite-dimensional representation of $\mathrm{SU}(2)$ on the vector space $W$. The space that generalizes to the case $\theta \neq 0$, the space of $\mathrm{SU}(2)$-equivariant maps from $S^{7}$ to $W$, is given by,

$$
C^{\infty}\left(S_{\theta^{\prime}}^{7}\right) \otimes_{\rho} W:=\left\{\eta \in C^{\infty}\left(S_{\theta^{\prime}}^{7}\right) \otimes W:\left(\alpha_{w} \otimes \mathrm{id}\right)(\eta)=\left(\mathrm{id} \otimes \rho(w)^{-1}\right)(\eta), \forall w \in \mathrm{SU}(2)\right\},
$$

where $\alpha_{w}$ is the $\mathrm{SU}(2)$ action given in (4.7). This space is clearly a $C^{\infty}\left(S_{\theta}^{4}\right)$-bimodule. We have proved in [27] that $C^{\infty}\left(S_{\theta^{\prime}}^{7}\right) \bigotimes_{\rho} W$ is a finite projective module. It is to be thought of as the module of sections of a "noncommutative vector bundle" associated to the principal bundle via the representation $\rho$. It is worth stressing that the choice of a projection for a finite projective module requires the choice of one of the two (left or right) module structures. Similarly, the definition of a Hermitian structure requires the choice of the left or right module structure. In the following, we will always work with the right structure for the associated modules. There is a natural (right) Hermitian structure on $C^{\infty}\left(S_{\theta^{\prime}}^{7}\right) \bigotimes_{\rho} W$, defined in terms of a fixed inner product of $W$ as,

$$
\left\langle\eta, \eta^{\prime}\right\rangle:=\sum_{i} \bar{\eta}_{i} \eta_{i}^{\prime}
$$

where we denoted $\eta=\sum_{i} \eta_{i} \otimes e_{i}$ and $\eta^{\prime}=\sum_{i} \eta_{i}^{\prime} \otimes e_{i}$, given an orthonormal basis $\left\{e_{i}, i=1, \cdots, \operatorname{dim} W\right\}$ of $W$. One quickly checks that $\left\langle\eta, \eta^{\prime}\right\rangle$ is an element in $C^{\infty}\left(S_{\theta}^{4}\right)$, and that $\langle$,$\rangle satisfies all conditions of a right Hermitian structure.$

The bimodules $C^{\infty}\left(S_{\theta^{\prime}}^{7}\right) \otimes_{\rho} W$ are of the type described in Section 3.3. The associated vector bundle $E=S^{7} \times_{\rho} W$ on $S^{4}$ carries an action $V$ of $\widetilde{T}^{2}$ induced from its action on $S^{7}$, which is obviously $\sigma$-equivariant. By the very definition of $C^{\infty}\left(S_{\theta^{\prime}}^{7}\right)$ and of $\Gamma^{\infty}\left(S_{\theta}^{4}, E\right)$ in Section 3.3, it follows that $C^{\infty}\left(S_{\theta^{\prime}}^{7}\right) \otimes_{\rho} W \simeq \Gamma^{\infty}\left(S_{\theta}^{4}, E\right)$. Indeed, from the undeformed isomorphism, $\Gamma^{\infty}\left(S^{4}, E\right) \simeq C^{\infty}\left(S^{7}\right) \bigotimes_{\rho} W$, the quantization map $L_{\theta^{\prime}}$ of $C^{\infty}\left(S^{7}\right)$, acting only on the first leg of the tensor product, establishes this isomorphism,

$$
L_{\theta^{\prime}}: C^{\infty}\left(S^{7}\right) \bigotimes_{\rho} W \rightarrow C^{\infty}\left(S_{\theta^{\prime}}^{7}\right) \bigotimes_{\rho} W .
$$


The above is well defined since the action of $\widetilde{\mathbb{T}}^{2}$ commutes with the action of $S U(2)$. Also, it is such that $L_{\theta^{\prime}}\left(f \triangleright_{\theta^{\prime}} \eta\right)=L_{\theta^{\prime}}(f) L_{\theta^{\prime}}(\eta)=L_{\theta}(f) L_{\theta^{\prime}}(\eta)$ for $f \in C^{\infty}\left(S^{4}\right)$ and $\eta \in C^{\infty}\left(S^{7}\right) \bigotimes_{\rho} W$, due to the identity $L_{\theta^{\prime}}=L_{\theta}$ on $C^{\infty}\left(S^{4}\right) \subset C^{\infty}\left(S^{7}\right)$. A similar result holds for the action $\triangleleft_{\theta^{\prime}}$.

Proposition 10. The right $C^{\infty}\left(S_{\theta}^{4}\right)$-modules $C^{\infty}\left(S_{\theta^{\prime}}^{7}\right) \bigotimes_{\rho} W$ admits a homogeneous module basis $\left\{e_{\alpha}, \alpha=1, \cdots, N\right\}$ - with a suitable $N$ - that is, under the action $V$ of the torus $\widetilde{\mathbb{T}}^{2}$, its elements transform as,

$$
V_{s}\left(e_{\alpha}\right)=e^{2 \pi i s \cdot r_{\alpha}} e_{\alpha}, \quad s \in \widetilde{\mathbb{T}}^{2} .
$$

with $r_{\alpha} \in \mathbb{Z}^{2}$ the degree of $e_{\alpha}$.

Proof. The vector space $W$ is a direct sum of irreducible representation spaces of $\mathrm{SU}(2)$ and the module $C^{\infty}\left(S_{\theta^{\prime}}^{7}\right) \bigotimes_{\rho} W$ decomposes accordingly. Thus, we can restrict to irreducible representations. The latter are labeled by an integer $n$ with $W \simeq \mathbb{C}^{n+1}$.

Consider first the case $W=\mathbb{C}^{2}$. Using [27, Proposition 2], a basis $\left\{e_{1}, \cdots, e_{4}\right\}$ of the right module $C^{\infty}\left(S_{\theta^{\prime}}^{7}\right) \bigotimes_{\rho} \mathbb{C}^{2}$ is given by the columns of $\Psi^{\dagger}$ where $\Psi$ is the matrix in (4.6):

$$
e_{1}:=\left(\begin{array}{c}
\psi_{1}^{*} \\
-\psi_{2}
\end{array}\right), \quad e_{2}:=\left(\begin{array}{c}
\psi_{2}^{*} \\
\psi_{1}
\end{array}\right), \quad e_{3}:=\left(\begin{array}{c}
\psi_{3}^{*} \\
-\psi_{4}
\end{array}\right), \quad e_{4}:=\left(\begin{array}{c}
\psi_{4}^{*} \\
\psi_{3}
\end{array}\right) .
$$

Using the explicit action (4.14) it is immediate to compute the corresponding degrees,

$$
r_{1}=(-1,0), \quad r_{2}=(1,0), \quad r_{3}=(0,1), \quad r_{4}=(0,-1) .
$$

More generally, with $W=\mathbb{C}^{n+1}$ a homogeneous basis $\left\{e_{\alpha}, \alpha=1, \cdots, 4^{n}\right\}$ for the right module $C^{\infty}\left(S_{\theta^{\prime}}^{7}\right) \otimes_{\rho} \mathbb{C}^{n+1}$ can be constructed from the columns of a similar $(n+1) \times 4^{n}$ matrix $\Psi_{(n)}^{\dagger}$ given in [27].

The above property allows us to prove a useful result for the associated modules.

Proposition 11. Let $\rho_{1}$ and $\rho_{2}$ be two finite dimensional representations of $\mathrm{SU}(2)$ on the vector spaces $W_{1}$ and $W_{2}$, respectively. There is the following isomorphism of right $C^{\infty}\left(S_{\theta}^{4}\right)$-modules,

$$
\left(C^{\infty}\left(S_{\theta^{\prime}}^{7}\right) \otimes_{\rho_{1}} W_{1}\right) \bar{\otimes}_{C^{\infty}\left(S_{\theta}^{4}\right)}\left(C^{\infty}\left(S_{\theta^{\prime}}^{7}\right) \bigotimes_{\rho_{2}} W_{2}\right) \simeq C^{\infty}\left(S_{\theta^{\prime}}^{7}\right) \bigotimes_{\rho_{1} \otimes \rho_{2}}\left(W_{1} \otimes W_{2}\right) .
$$

Proof. Let $\left\{e_{\alpha}^{1}, \alpha=1, \cdots, N_{1}\right\}$ and $\left\{e_{\beta}^{2}, \beta=1, \cdots, N_{2}\right\}$ be homogeneous bases for the right modules $C^{\infty}\left(S_{\theta^{\prime}}^{7}\right) \bigotimes_{\rho_{1}} W_{1}$ and $C^{\infty}\left(S_{\theta^{\prime}}^{7}\right) \bigotimes_{\rho_{2}} W_{2}$ respectively. Then, the right module $C^{\infty}\left(S_{\theta^{\prime}}^{7}\right) \otimes_{\rho_{1} \otimes \rho_{2}}\left(W_{1} \otimes W_{2}\right)$ has a homogeneous basis given by $\left\{e_{\alpha}^{1} \otimes e_{\beta}^{2}\right\}$. We define a map

$$
\phi:\left(C^{\infty}\left(S_{\theta^{\prime}}^{7}\right) \otimes_{\rho_{1}} W_{1}\right) \bar{\otimes}_{C^{\infty}\left(S_{\theta}^{4}\right)}\left(C^{\infty}\left(S_{\theta^{\prime}}^{7}\right) \bigotimes_{\rho_{2}} W_{2}\right) \rightarrow C^{\infty}\left(S_{\theta^{\prime}}^{7}\right) \otimes_{\rho_{1} \otimes \rho_{2}}\left(W_{1} \otimes W_{2}\right)
$$

by

$$
\phi\left(e_{\alpha}^{1} \times_{\theta} f_{\alpha}^{1} \otimes e_{\beta}^{2} \times_{\theta} f_{\beta}^{2}\right)=\left(e_{\alpha}^{1} \otimes e_{\beta}^{2}\right) \times_{\theta} \sigma_{r_{\beta} \theta}\left(f_{\alpha}^{1}\right) \times_{\theta} f_{\beta}^{2},
$$

with summation over $\alpha$ and $\beta$ understood. Here $r_{\beta} \in \mathbb{Z}^{2}$ is the degree of $e_{\beta}^{2}$ under the action of $\widetilde{\mathbb{T}}^{2}$, so that $e_{\beta}^{2} \times_{\theta} \sigma_{r_{\beta} \theta}(f)=f \times_{\theta} e_{\beta}^{2}$ for any $f \in C^{\infty}\left(S_{\theta}^{4}\right)$. Note that this map is well-defined since

$$
\phi\left(e_{\alpha}^{1} \times_{\theta} f_{\alpha}^{1} \times_{\theta} f \otimes e_{\beta}^{2} \times_{\theta} f_{\beta}^{2}-e_{\alpha}^{1} \times_{\theta} f_{\alpha}^{1} \otimes f \times_{\theta} e_{\beta}^{2} \times_{\theta} f_{\beta}^{2}\right)=0 .
$$


Moreover, it is clearly a map of right $C^{\infty}\left(S_{\theta}^{4}\right)$-modules. In fact, it is an isomorphism with its inverse given explicitly by

$$
\phi^{-1}\left(\left(e_{\alpha}^{1} \otimes e_{\beta}^{2}\right) \times_{\theta} f_{\alpha \beta}\right)=e_{1}^{1} \otimes\left(e_{\beta}^{2} \times_{\theta} f_{1 \beta}\right)+\cdots+e_{N_{1}}^{1} \otimes\left(e_{\beta}^{2} \times_{\theta} f_{N_{1} \beta}\right)
$$

with $f_{\alpha \beta} \in C^{\infty}\left(S_{\theta}^{4}\right)$.

Given a right $C^{\infty}\left(S_{\theta}^{4}\right)$-module $\mathcal{E}$, its dual module is defined by

$$
\mathcal{E}^{\prime}:=\left\{\phi: \mathcal{E} \rightarrow C^{\infty}\left(S_{\theta}^{4}\right): \phi(\eta f)=\phi(\eta) f, \forall f \in C^{\infty}\left(S_{\theta}^{4}\right)\right\}
$$

and is naturally a left $C^{\infty}\left(S_{\theta}^{4}\right)$-module. In the case that $\mathcal{E}$ is also a left $C^{\infty}\left(S_{\theta}^{4}\right)$-module, then $\mathcal{E}^{\prime}$ is also a right $C^{\infty}\left(S_{\theta}^{4}\right)$-module. If $\mathcal{E}:=C^{\infty}\left(S_{\theta^{\prime}}^{7} \otimes_{\rho} W\right.$ comes from the $\mathrm{SU}(2)$ representation $(W, \rho)$, by using the induced dual representation $\rho^{\prime}$ on the dual vector space $W^{\prime}$ given by

$$
\left(\rho^{\prime}(w) v^{\prime}\right)(v):=v^{\prime}\left(\rho(w)^{-1} v\right) ; \quad \forall v^{\prime} \in W^{\prime}, v \in W,
$$

we have that

$$
\begin{aligned}
\mathcal{E}^{\prime} & \simeq C^{\infty}\left(S_{\theta^{\prime}}^{7}\right) \otimes_{\rho^{\prime}} W^{\prime} \\
& :=\left\{\phi \in C^{\infty}\left(S_{\theta^{\prime}}^{7}\right) \otimes W^{\prime}:\left(\alpha_{w} \otimes \mathrm{id}\right)(\phi)=\left(\mathrm{id} \otimes \rho^{\prime}(w)^{-1}\right)(\phi), \forall w \in \mathrm{SU}(2)\right\} .
\end{aligned}
$$

Next, let $L(W)$ denote the space of linear maps on $W$, so that $L(W)=W \otimes W^{\prime}$. The adjoint action of $\mathrm{SU}(2)$ on $L(W)$ is the tensor product representation ad $:=\rho \otimes \rho^{\prime}$ on $W \otimes W^{\prime}$. We define

$$
\begin{aligned}
C^{\infty}\left(S_{\theta^{\prime}}^{7}\right) \bigotimes_{\mathrm{ad}} L(W):=\left\{T \in C^{\infty}\left(S_{\theta^{\prime}}^{7}\right) \otimes L(W):\right. \\
\left.\quad:\left(\alpha_{w} \otimes \mathrm{id}\right)(T)=\left(\operatorname{id} \otimes \operatorname{ad}(w)^{-1}\right)(T), \forall w \in \mathrm{SU}(2)\right\},
\end{aligned}
$$

and write $T=T_{i j} \otimes e_{i j}$ with respect to the basis $\left\{e_{i j}\right\}$ of $L(W)$ induced from the basis $\left\{e_{i}\right\}_{i=1}^{\operatorname{dim} W}$ of $W$ and the dual one $\left\{e_{i}^{\prime}\right\}_{i=1}^{\operatorname{dim} W}$ of $W^{\prime}$.

On the other hand, there is the endomorphism algebra

$$
\operatorname{End}_{C^{\infty}\left(S_{\theta}^{4}\right)}(\mathcal{E}):=\left\{T: \mathcal{E} \rightarrow \mathcal{E}: T(\eta f)=T(\eta) f, \forall f \in C^{\infty}\left(S_{\theta}^{4}\right)\right\} .
$$

We will suppress the subscript $C^{\infty}\left(S_{\theta}^{4}\right)$ from End in the following. As a corollary to the previous Proposition, we have the following.

Proposition 12. Let $\mathcal{E}:=C^{\infty}\left(S_{\theta^{\prime}}^{7}\right) \bigotimes_{\rho} W$ for a finite-dimensional representation $\rho$. Then there is an isomorphism of algebras

$$
\operatorname{End}(\mathcal{E}) \simeq C^{\infty}\left(S_{\theta^{\prime}}^{7}\right) \bigotimes_{\mathrm{ad}} L(W)
$$

Proof. By Proposition 11, we have that $C^{\infty}\left(S_{\theta^{\prime}}^{7}\right) \otimes_{\mathrm{ad}} L(W) \equiv C^{\infty}\left(S_{\theta^{\prime}}^{7}\right) \otimes_{\rho \otimes \rho^{\prime}}\left(W \otimes W^{\prime}\right)$ is isomorphic to $\mathcal{E} \bar{\otimes}_{C^{\infty}\left(S_{\theta}^{4}\right)} \mathcal{E}^{\prime}$ as a right $C^{\infty}\left(S_{\theta}^{4}\right)$-module. Since $\mathcal{E}$ is a finite projective $C^{\infty}\left(S_{\theta}^{4}\right)$-module, there is an isomophism $\operatorname{End}(\mathcal{E}) \simeq \mathcal{E} \bar{\otimes}_{C^{\infty}\left(S_{\theta}^{4}\right)} \mathcal{E}^{\prime}$, whence the result. 
We see that the algebra of endomorphisms of the module $\mathcal{E}$ can be understood as the space of sections of the noncommutative vector bundle associated to the adjoint representation on $L(W)$ - exactly as it happens in the classical case. This also allows an identification of skew-hermitian endomorphisms $\operatorname{End}^{s}(\mathcal{E})$ - which were defined in general in (2.19) - for the toric deformations at hand.

Corollary 13. There is an identification

$$
\operatorname{End}^{s}(\mathcal{E}) \simeq C_{\mathbb{R}}^{\infty}\left(S_{\theta^{\prime}}^{7}\right) \bigotimes_{\mathrm{ad}} u(n),
$$

with $C_{\mathbb{R}}^{\infty}\left(S_{\theta^{\prime}}^{7}\right)$ denoting the subspace of self-adjoint elements in $C^{\infty}\left(S_{\theta^{\prime}}^{7}\right)$ and $u(n)$ consists of skew-adjoint matrices in $M_{n}(\mathbb{C}) \simeq L(W)$, with $n=\operatorname{dim} W$.

Proof. Note that the involution $T \mapsto T^{*}$ in $\operatorname{End}(\mathcal{E})$ reads in components $T_{i j} \mapsto \overline{T_{j i}}$ so that, with the identification of Proposition 12, the space $\operatorname{End}^{s}(\mathcal{E})$ is made of elements $X \in C^{\infty}\left(S_{\theta^{\prime}}^{7}\right) \bigotimes_{\mathrm{ad}} L(W)$ satisfying $\overline{X_{j i}}=-X_{i j}$. Since any element in $C^{\infty}\left(S_{\theta^{\prime}}^{7}\right)$ can be written as the sum of two self-adjoint elements, $X_{i j}=X_{i j}^{\Re}+i X_{i j}^{\Im}$, we can write

$$
X=\sum_{i} X_{i i}^{\Im} \otimes i e_{i i}+\sum_{i \neq j} X_{i j}^{\Re} \otimes\left(e_{i j}-e_{j i}\right)+X_{i j}^{\Im} \otimes\left(i e_{i j}+i e_{j i}\right)=\sum_{a} X_{a} \otimes \sigma^{a},
$$

with $X_{a}$ generic elements in $C_{\mathbb{R}}^{\infty}\left(S_{\theta^{\prime}}^{7}\right)$ and $\left\{\sigma^{a}, a=1, \ldots, n^{2}\right\}$ the generators of $u(n)$.

Example 14. Of central interest in the following is the special case of the noncommutative instanton bundle first constructed in [15]. Now $W=\mathbb{C}^{2}$ and $\rho$ is the defining representation of $\mathrm{SU}(2)$. The projection $p$ giving the $C^{\infty}\left(S_{\theta}^{4}\right)$-module $C^{\infty}\left(S_{\theta^{\prime}}^{7}\right) \bigotimes_{\rho} \mathbb{C}^{2}$ as a direct summand of $\left(C^{\infty}\left(S_{\theta}^{4}\right)\right)^{N}$ for some $N$, is precisely given by the one in (4.8) and $N=4$. Indeed, a generic element in $C^{\infty}\left(S_{\theta^{\prime}}^{7}\right) \bigotimes_{\rho} \mathbb{C}^{2}$ is of the form $\Psi^{\dagger} f$ for some $f \in C^{\infty}\left(S_{\theta}^{4}\right) \otimes \mathbb{C}^{4}$ with $\Psi$ defined in (4.6), and the correspondence is given by

$$
C^{\infty}\left(S_{\theta^{\prime}}^{7}\right) \bigotimes_{\rho} \mathbb{C}^{2} \simeq p\left(C^{\infty}\left(S_{\theta}^{4}\right)\right)^{4}, \quad \Psi^{\dagger} f \leftrightarrow p f .
$$

Furthermore, $\operatorname{End}(\mathcal{E}) \simeq C^{\infty}\left(S_{\theta^{\prime}}^{7}\right) \bigotimes_{\mathrm{ad}} M_{2}(\mathbb{C})$. It is a known fact that $M_{2}(\mathbb{C})$ decomposes into the adjoint representation su(2) and the trivial representation $\mathbb{C}$ while it is easy to see that $C^{\infty}\left(S_{\theta^{\prime}}^{7}\right) \bigotimes_{\mathrm{id}} \mathbb{C} \simeq C^{\infty}\left(S_{\theta}^{4}\right)$. Thus, we conclude that

$$
\operatorname{End}(\mathcal{E}) \simeq \Gamma^{\infty}\left(\operatorname{ad}\left(S_{\theta^{\prime}}^{7}\right)\right) \oplus C^{\infty}\left(S_{\theta}^{4}\right)
$$

where we have set $\Gamma^{\infty}\left(\operatorname{ad}\left(S_{\theta^{\prime}}^{7}\right)\right):=C^{\infty}\left(S_{\theta^{\prime}}^{7}\right) \bigotimes_{\mathrm{ad}} s u(2)$. The latter $C^{\infty}\left(S_{\theta}^{4}\right)$-bimodule will be understood as the space of (complex) sections of the adjoint bundle. It is the complexification of the traceless skew-hermitian endomorphisms $C_{\mathbb{R}}^{\infty}\left(S_{\theta^{\prime}}^{7}\right) \bigotimes_{\mathrm{ad}} s u(2)$.

\subsection{Yang-Mills theory on $S_{\theta}^{4}$}

Let us now move to the main goal of this paper and discuss the Yang-Mills action functional on $S_{\theta}^{4}$ together with its equations of motion. We will see that instantons naturally arise as the local minima of this action.

Before we proceed we recall the noncommutative spin structure $\left(C^{\infty}\left(S_{\theta}^{4}\right), \mathcal{H}, D, \gamma_{5}\right)$ of $S_{\theta}^{4}$ with $\mathcal{H}=L^{2}\left(S^{4}, \mathcal{S}\right)$ the Hilbert space of spinors, $D$ the undeformed Dirac operator, and $\gamma_{5}$ - the even structure - the fifth Dirac matrix. 
Let $\mathcal{E}=\Gamma^{\infty}\left(S_{\theta}^{4}, E\right)$ for some $\sigma$-equivariant vector bundle $E$ on $S^{4}$, so that there exists a projection $p \in M_{N}\left(C^{\infty}\left(S_{\theta}^{4}\right)\right)$ such that $\mathcal{E} \simeq p\left(C^{\infty}\left(S_{\theta}^{4}\right)^{N}\right.$. Recall from Section 2 that a connection $\nabla$ on $\mathcal{E}=\Gamma^{\infty}\left(S_{\theta}^{4}, E\right)$ can be given as a map from $\mathcal{E}$ to $\mathcal{E} \otimes \Omega^{1}\left(S_{\theta}^{4}\right)$ satisfying a Leibniz rule with respect to the right multiplication of $C^{\infty}\left(S_{\theta}^{4}\right)$ on $\mathcal{E}$. It is also required to be compatible with a Hermitian structure on $\mathcal{E}$. The Yang-Mills action functional is defined in terms of the curvature $F$ of $\nabla$, an element in $\operatorname{Hom}_{C}{ }_{\left(S_{\theta}^{4}\right)}\left(\mathcal{E}, \mathcal{E} \otimes \Omega^{2}\left(S_{\theta}^{4}\right)\right)$. Equivalently, it can be thought of as belonging to $\operatorname{End}_{\Omega\left(S_{\theta}^{4}\right)}\left(\mathcal{E} \otimes \Omega\left(S_{\theta}^{4}\right)\right)$ of degree 2. We define an inner product on the latter algebra following [12, III.3]. Any $T \in \operatorname{End}_{\Omega\left(S_{\theta}^{4}\right)}(\mathcal{E} \otimes$ $\left.\Omega\left(S_{\theta}^{4}\right)\right)$ of degree $k$ can be also understood as an element in $p M_{N}\left(\Omega^{k}\left(S_{\theta}^{4}\right)\right) p$, since $\mathcal{E} \otimes \Omega\left(S_{\theta}^{4}\right)$ is a finite projective module over $\Omega\left(S_{\theta}^{4}\right)$. A trace over internal indices together with the inner product in (3.25), defines the inner product $(\cdot, \cdot)_{2}$ on $\operatorname{End}_{\Omega\left(S_{\theta}^{4}\right)}\left(\mathcal{E} \otimes \Omega\left(S_{\theta}^{4}\right)\right)$. In particular, we can give the following definition:

Definition 15. The Yang-Mills action functional on the collection $C(\mathcal{E})$ of compatible connections on $\mathcal{E}$ is defined by

$$
\mathrm{YM}(\nabla)=(F, F)_{2}=f *_{\theta} \operatorname{tr}\left(F *_{\theta} F\right)
$$

for any connection $\nabla$ with curvature $F$.

Recall from Section 2 that gauge transformations are unitary endomorphisms $\mathcal{U}(\mathcal{E})$ of $\mathcal{E}$.

Lemma 16. The Yang-Mills action functional is gauge invariant, positive and quartic.

Proof. From equation (2.26), under a gauge transformation $u \in \mathcal{U}(\mathcal{E})$ the curvature $F$ transforms as $F \mapsto u^{*} F u$. Since $\mathcal{U}(\mathcal{E})$ can be identified with the unitary elements in $p M_{N}(\mathcal{A}) p$, it follows that

$$
\mathrm{YM}\left(\nabla^{u}\right)=f \sum_{i, j, k, l} *_{\theta}\left(\overline{u_{j i}} F_{j k} *_{\theta} F_{k l} u_{l i}\right)=\mathrm{YM}(\nabla),
$$

using the tracial property of the Dixmier trace and the fact that $u_{l i} \overline{u_{j i}}=\delta_{l j}$.

Positiveness of the Yang-Mills action functional follows from Lemma 7 giving

$$
(F, F)_{2}=\left(F_{D}, F_{D}\right)_{D}=f F_{D}^{*} F_{D}
$$

which is clearly positive.

The Yang-Mills equations of motion (equations for critical points) are obtained from the Yang-Mills action functional by a variational principle. Let us describe how this principle works in our case. We consider a linear perturbation $\nabla_{t}=\nabla+t \alpha$ of a connection $\nabla$ on $\mathcal{E}$ by an element $\alpha \in \operatorname{Hom}\left(\mathcal{E}, \mathcal{E} \otimes_{C^{\infty}\left(S_{\theta}^{4}\right)} \Omega^{1}\left(S_{\theta}^{4}\right)\right)$. The curvature $F_{t}$ of $\nabla_{t}$ is readily computed as $F_{t}=F+t[\nabla, \alpha]+\mathcal{O}\left(t^{2}\right)$. If we suppose that $\nabla$ is a critical point of the Yang-Mills action functional, this linear perturbation should not affect the action. In other words, we need

$$
\left.\frac{\partial}{\partial t}\right|_{t=0} \operatorname{YM}\left(\nabla_{t}\right)=0
$$


If we substitute the explicit formula for $F_{t}$, we obtain

$$
([\nabla, \alpha], F)_{2}+\overline{([\nabla, \alpha], F)_{2}}=0
$$

using the fact that $(\cdot, \cdot)_{2}$ defines a complex scalar product on $\operatorname{Hom}\left(\mathcal{E}, \mathcal{E} \otimes \Omega\left(S_{\theta}^{4}\right)\right)$. Positive definiteness of this scalar product implies that $\left(F_{t}, F_{t}\right)=\overline{\left(F_{t}, F_{t}\right)}$, which when differentiated with respect to $t$, at $t=0$, yields $([\nabla, \alpha], F)_{2}=\overline{([\nabla, \alpha], F)_{2}}$; hence, $([\nabla, \alpha], F)_{2}=0$. Using the fact that $\alpha$ was arbitrary, we derive the following equations of motion

$$
\left[\nabla^{*}, F\right]=0 .
$$

where the adjoint $\left[\nabla^{*}, \cdot\right]$ is defined with respect to the inner product $(\cdot, \cdot)_{2}$, i.e.

$$
\left(\left[\nabla^{*}, \alpha\right], \beta\right)_{2}=(\alpha,[\nabla, \beta])_{2}
$$

for any $\alpha \in \operatorname{Hom}\left(\mathcal{E}, \mathcal{E} \otimes \Omega^{3}\left(S_{\theta}^{4}\right)\right)$ and any $\beta \in \operatorname{Hom}\left(\mathcal{E}, \mathcal{E} \otimes \Omega^{1}\left(S_{\theta}^{4}\right)\right)$. From Lemma 8 , it follows that $\left[\nabla^{*}, F\right]=*_{\theta}\left[\nabla, *_{\theta} F\right]$, so that the equations of motion can be written as the more familiar Yang-Mills equations,

$$
\left[\nabla, *_{\theta} F\right]=0 .
$$

Clearly, connections with a self-dual or antiself-dual curvature, $*_{\theta} F= \pm F$, are special solutions of the Yang-Mills equations. Indeed, in this case the latter equations follow directly from the Bianchi identity, $[\nabla, F]=0$, given in Proposition 1 .

We will now establish a connection between the Yang-Mills action functional and the so-called topological action [12, VI.3] on $S_{\theta}^{4}$. Suppose $\mathcal{E}$ is a finite projective module over $C^{\infty}\left(S_{\theta}^{4}\right)$ defined by a projection $p \in M_{N}\left(C^{\infty}\left(S_{\theta}^{4}\right)\right)$. The topological action for $\mathcal{E}$ is the pairing between the class of $p$ in K-theory and the cyclic cohomology of $C^{\infty}\left(S_{\theta}^{4}\right)$. For computational purposes, we define it in terms of the curvature of a connection on $\mathcal{E}$,

Definition 17. Let $\nabla$ be a connection on $\mathcal{E}$ with curvature $F$. The topological action is given by

$$
\operatorname{Top}(\mathcal{E})=\left(F, *_{\theta} F\right)_{2}=f *_{\theta} \operatorname{tr}\left(F^{2}\right)
$$

where the trace is taken over internal indices and in the second equality we have used the identity $*_{\theta} \circ *_{\theta}=\mathrm{id}$ on $S_{\theta}^{4}$.

Let us show that this does not depend on the choice of a connection on $\mathcal{E}$. Since two connections differ by an element $\alpha$ in $\operatorname{Hom}_{C^{\infty}\left(S_{\theta}^{4}\right)}\left(\mathcal{E}, \mathcal{E} \otimes \Omega^{1}\left(S_{\theta}^{4}\right)\right)$, we have to establish that $\left(F^{\prime}, *_{\theta} F^{\prime}\right)_{2}=\left(F, *_{\theta} F\right)_{2}$ where $F^{\prime}=F+t[\nabla, \alpha]+\mathcal{O}\left(t^{2}\right)$ is the curvature of $\nabla^{\prime}:=\nabla+t \alpha$, $t \in \mathbb{R}$. By definition of the inner product $(\cdot, \cdot)_{2}$ we have that

$$
\begin{aligned}
\left(F^{\prime}, *_{\theta} F^{\prime}\right)_{2}-\left(F, *_{\theta} F\right)_{2} & =t\left(F, *_{\theta}[\nabla, \alpha]\right)_{2}+t\left([\nabla, \alpha], *_{\theta} F\right)_{2}+\mathcal{O}\left(t^{2}\right) \\
& =t\left(F,\left[\nabla^{*}, *_{\theta} \alpha\right]\right)_{2}+t\left(\left[\nabla^{*}, *_{\theta} \alpha\right], F\right)_{2}+\mathcal{O}\left(t^{2}\right) .
\end{aligned}
$$

With equation (4.31), this reduces to

$$
\left(F^{\prime}, *_{\theta} F^{\prime}\right)_{2}-\left(F, *_{\theta} F\right)_{2}=t\left([\nabla, F], *_{\theta} \alpha\right)_{2}+t\left(*_{\theta} \alpha,[\nabla, F]\right)_{2}+\mathcal{O}\left(t^{2}\right)
$$


which vanishes modulo $t^{2}$ due to the Bianchi identity $[\nabla, F]=0$.

The Hodge star operator $*_{\theta}$ splits $\Omega^{2}\left(S_{\theta}^{4}\right)$ into a self-dual and antiself-dual space,

$$
\Omega^{2}\left(S_{\theta}^{4}\right)=\Omega_{+}^{2}\left(S_{\theta}^{4}\right) \oplus \Omega_{-}^{2}\left(S_{\theta}^{4}\right) .
$$

In fact, $\Omega_{ \pm}^{2}\left(S_{\theta}^{4}\right)=L_{\theta}\left(\Omega_{ \pm}^{2}\left(S^{4}\right)\right)$. This decomposition is orthogonal with respect to the inner product $(\cdot, \cdot)_{2}$ a fact that follows from the property $(\alpha, \beta)_{2}=\overline{(\beta, \alpha)_{2}}$; thus we can write the Yang-Mills action functional as

$$
\mathrm{YM}(\nabla)=\left(F_{+}, F_{+}\right)_{2}+\left(F_{-}, F_{-}\right)_{2} .
$$

Comparing this with the analogue decomposition of the topological action,

$$
\operatorname{Top}(\mathcal{E})=\left(F_{+}, F_{+}\right)_{2}-\left(F_{-}, F_{-}\right)_{2},
$$

we see that $\operatorname{YM}(\nabla) \geq|\operatorname{Top}(\mathcal{E})|$, with equality holding iff

$$
*_{\theta} F= \pm F \text {. }
$$

Solutions of these equations are called instantons. We conclude that instantons are absolute minima of the Yang-Mills action functional.

\section{Construction of $\mathrm{SU}(2)$-instantons on $S_{\theta}^{4}$}

In this section, we construct a set of $\mathrm{SU}(2)$ instantons on $S_{\theta}^{4}$, with topological charge 1 , by acting with a twisted infinitesimal conformal symmetry on the basic instanton on $S_{\theta}^{4}$ constructed in [15]. We will find a five-parameter family of such instantons. Then we prove that the "tangent space" to the moduli space of irreducible instantons at the basic instanton is five-dimensional, proving that this set is complete. Here, one has to be careful with the notion of tangent space to the moduli space. As will be discussed elsewhere [26], one can construct a noncommutative family of instantons, that is instantons parametrized by the quantum quotient space of the deformed conformal group $\mathrm{SL}_{\theta}(2, \mathbb{H})$ by the deformed spin group $\operatorname{Sp}_{\theta}(2)$. It turns out that the basic instanton of [15] is a "classical point" in this moduli space of instantons. We perturb the connection $\nabla_{0}$ linearly by sending $\nabla_{0} \mapsto \nabla_{0}+t \alpha$ where $t \in \mathbb{R}$ and $\alpha \in \operatorname{Hom}\left(\mathcal{E}, \mathcal{E} \otimes \Omega^{1}\left(S_{\theta}^{4}\right)\right)$. In order for this new connection still to be an instanton, we have to impose the self-duality equations on its curvature. After deriving this equation with respect to $t$, setting $t=0$ afterwards, we obtain the linearized self-duality equations to be fulfilled by $\alpha$. It is in this sense that we are considering the tangent space to the moduli space of instantons at the point $\nabla_{0}$.

\subsection{The basic instanton}

We start with a technical lemma that simplifies the discussion. Let $\mathcal{E}=C^{\infty}\left(S_{\theta^{\prime}}^{7}\right) \bigotimes_{\rho} W$ be a module of sections associated to a finite dimensional representation of $\mathrm{SU}(2)$, as defined in equation (4.15).

Lemma 18. There is the following isomorphism of right $C^{\infty}\left(S_{\theta}^{4}\right)$-modules,

$$
\mathcal{E} \otimes_{C^{\infty}\left(S_{\theta}^{4}\right)} \Omega\left(S_{\theta}^{4}\right) \simeq \Omega\left(S_{\theta}^{4}\right) \otimes_{C^{\infty}\left(S_{\theta}^{4}\right)} \mathcal{E} .
$$

Consequently, $\operatorname{Hom}\left(\mathcal{E}, \mathcal{E} \otimes_{C^{\infty}\left(S_{\theta}^{4}\right)} \Omega\left(S_{\theta}^{4}\right)\right) \simeq \Omega\left(S_{\theta}^{4}\right) \otimes_{C^{\infty}\left(S_{\theta}^{4}\right)} \operatorname{End}(\mathcal{E})$. 
Proof. Recall from Proposition 10 that the right $C^{\infty}\left(S_{\theta}^{4}\right)$-module $\mathcal{E}$ has a homogeneous module-basis $\left\{e_{\alpha}, \alpha=1, \cdots, N\right\}$ for some $N$ and each $e_{\alpha}$ of degree $r_{\alpha}$. A generic element in $\mathcal{E} \otimes_{C^{\infty}\left(S_{\theta}^{4}\right)} \Omega\left(S_{\theta}^{4}\right)$ can be written as a sum $\sum_{\alpha} e_{\alpha} \otimes_{C^{\infty}\left(S_{\theta}^{4}\right)} \omega^{\alpha}$, with $\omega^{\alpha}$ an element in $\Omega\left(S_{\theta}^{4}\right)$. Now, for every $\omega \in \Omega\left(S_{\theta}^{4}\right)$ there is an element $\widetilde{\omega} \in \Omega\left(S_{\theta}^{4}\right)$ - given explicitly by $\widetilde{\omega}=\sigma_{r_{\alpha} \cdot \theta}(\omega)$ - such that $e_{\alpha} \omega=\widetilde{\omega} e_{\alpha}$ where the latter equality holds inside the algebra $\Omega\left(S_{\theta^{\prime}}^{7}\right)$ (recall that $\Omega\left(S_{\theta}^{4}\right) \subset \Omega\left(S_{\theta^{\prime}}^{7}\right)$ ). We can thus define a map

$$
T: \mathcal{E} \otimes_{C^{\infty}\left(S_{\theta}^{4}\right)} \Omega\left(S_{\theta}^{4}\right) \simeq \Omega\left(S_{\theta}^{4}\right) \otimes_{C^{\infty}\left(S_{\theta}^{4}\right)} \mathcal{E}
$$

by $T\left(e_{\alpha} \otimes_{C^{\infty}\left(S_{\theta}^{4}\right)} \omega^{\alpha}\right)=\widetilde{\omega}^{\alpha} \otimes e_{\alpha}$; it is a right $C^{\infty}\left(S^{4}\right)$-module map:

$$
T\left(e_{\alpha} \otimes_{C^{\infty}\left(S_{\theta}^{4}\right)}\left(\omega^{\alpha} \times_{\theta} f\right)\right)=\left(\widetilde{\omega}^{\alpha} \times_{\theta} \widetilde{f}\right) \otimes_{C^{\infty}\left(S_{\theta}^{4}\right)} e_{\alpha}=T\left(e_{\alpha} \otimes_{C^{\infty}\left(S_{\theta}^{4}\right)} \omega^{\alpha}\right) \times_{\theta} f .
$$

Since an inverse map $T^{-1}$ is easily constructed, $T$ gives the desired isomorphism.

Thus, we can unambiguously use the notation $\Omega\left(S_{\theta}^{4}, \mathcal{E}\right)$ for the above right $C^{\infty}\left(S_{\theta}^{4}\right)$ module $\mathcal{E} \otimes_{C^{\infty}\left(S_{\theta}^{4}\right)} \Omega\left(S_{\theta}^{4}\right)$.

We let $\nabla_{0}=p \circ \mathrm{d}$ be the canonical (Grassmann) connection on the projective module $\mathcal{E}=C^{\infty}\left(S_{\theta^{\prime}}^{7}\right) \bigotimes_{\rho} \mathbb{C}^{2} \simeq p\left(C^{\infty}\left(S_{\theta}^{4}\right)\right)^{4}$, with the projection $p=\Psi^{\dagger} \Psi$ of (4.8) and $\Psi$ is the matrix (4.6) (refer to Example 14). When acting on equivariant maps, we write $\nabla_{0}$ as

$$
\nabla_{0}: \mathcal{E} \rightarrow \mathcal{E} \otimes_{C^{\infty}\left(S_{\theta}^{4}\right)} \Omega^{1}\left(S_{\theta}^{4}\right), \quad\left(\nabla_{0} f\right)_{i}=\mathrm{d} f_{i}+\omega_{i j} \times_{\theta} f_{j}
$$

where $\omega$ - referred to as the gauge potential - is given in terms of the matrix $\Psi$ by

$$
\omega=\Psi^{\dagger} \mathrm{d} \Psi
$$

The above, is a $2 \times 2$-matrix with entries in $\Omega^{1}\left(S_{\theta^{\prime}}^{7}\right)$ satisfying $\overline{\omega_{i j}}=\omega_{j i}$ and $\sum_{i} \omega_{i i}=0$. Note here that the entries $\omega_{i j}$ commute with all elements in $C^{\infty}\left(S_{\theta^{\prime}}^{7}\right)$. Indeed, from (4.6) we see that the elements in $\omega_{i j}$ are $\mathbb{T}^{2}$-invariant and hence central (as one forms) in $\Omega\left(S_{\theta^{\prime}}^{7}\right)$. In other words $L_{\theta}(\omega)=\omega$, which shows that for an element $f \in \mathcal{E}$ as above, we have $\nabla_{0}(f)_{i}=\mathrm{d} f_{i}+\omega_{i j} \times_{\theta} f_{j}=\mathrm{d} f_{i}+\omega_{i j} f_{j}$ which coincides with the action of the classical connection $\mathrm{d}+\omega$ on $f$.

The curvature $F_{0}=\nabla_{0}^{2}=\mathrm{d} \omega+\omega^{2}$ of $\nabla_{0}$ is an element of $\operatorname{End}(\mathcal{E}) \otimes_{C^{\infty}\left(S_{\theta}^{4}\right)} \Omega^{2}\left(S_{\theta}^{4}\right)$ that satisfies [1, 14] the self-duality equation

$$
*_{\theta} F_{0}=F_{0}
$$

hence this connection is an instanton. At the classical value of the deformation parameter, $\theta=0$, the connection (5.2) is nothing but the $S U(2)$ instanton of [7].

Its "topological charge", i.e. the values of $\operatorname{Top}(\mathcal{E})$ in Definition [17, was already computed in [15]. Clearly it depends only on the class $[p]$ of the bundle and can be evaluated as the index

$$
\operatorname{Top}([p])=\operatorname{index}\left(D_{p}\right)=f \gamma_{5} \pi_{D}\left(\operatorname{ch}_{2}(p)\right)
$$

of the twisted Dirac operator

$$
D_{p}=p\left(D \otimes \mathbb{I}_{4}\right) p
$$


The last equality in (5.4) follows from the vanishing of the class $\operatorname{ch}_{1}(p)$ of the bundle 2 . On the other hand, one finds

$$
\left.\pi_{D}\left(\operatorname{ch}_{2}(p)\right)\right)=3 \gamma_{5}
$$

which, together with the fact that

$$
f 1=\operatorname{Tr}_{\omega}|D|^{-4}=\frac{1}{3}
$$

on $S^{4}$ (see for instance [22, 24]), gives the value $\operatorname{Top}([p])=1$.

We aim at constructing all connections $\nabla$ on $\mathcal{E}$ whose curvature satisfies the selfduality equations and have topological charge equal to 1 . We can write any such connection in terms of the canonical connection as in equation (2.9), i.e. $\nabla=\nabla_{0}+\alpha$ with $\alpha$ a one-form valued endomorphism of $\mathcal{E}$. Clearly, this will not change the value of the topological charge. Being interested in $\mathrm{SU}(2)$-instantons, we impose that $\alpha$ is traceless and skew-hermitian. Here the trace is taken in the second leg of $\operatorname{End}(\mathcal{E}) \simeq C^{\infty}\left(S_{\theta^{\prime}}^{7}\right) \bigotimes_{\mathrm{ad}} M_{2}(\mathbb{C})$. When complexified, we get an element $\alpha \in \Omega^{1}\left(S_{\theta}^{4}\right) \otimes_{C^{\infty}\left(S_{\theta}^{4}\right)} \Gamma^{\infty}\left(\operatorname{ad}\left(S_{\theta^{\prime}}^{7}\right)\right)=: \Omega^{1}\left(\operatorname{ad}\left(S_{\theta}^{4}\right)\right)$ (cf. Example 14).

As usual, we impose an irreducibility condition on the instanton connections, a connection on $\mathcal{E}$ being irreducible if it cannot be written as the sum of two other connections on $\mathcal{E}$. We are interested only in the irreducible instanton connections on the module $\mathcal{E}$.

Remark 19. In [27], we constructed projections $p_{(n)}$ for all modules $C^{\infty}\left(S_{\theta^{\prime}}^{7}\right) \bigotimes_{\rho} \mathbb{C}^{n}$ over $C^{\infty}\left(S_{\theta}^{4}\right)$ associated to the irreducible representations $\mathbb{C}^{n}$ of $\mathrm{SU}(2)$. The induced Grassmann connections $\nabla_{0}^{(n)}:=p_{(n)} \mathrm{d}$, when acting on $C^{\infty}\left(S_{\theta^{\prime}}^{7}\right) \bigotimes_{\rho} \mathbb{C}^{n}$, were written as $\mathrm{d}+\omega_{(n)}$, with $\omega_{(n)}$ an $n \times n$ matrix with entries in $\Omega^{1}\left(S_{\theta^{\prime}}^{7}\right)$. A similar argument as above then shows that all $\omega_{(n)}$ have entries that are central (as one forms) in $\Omega\left(S_{\theta^{\prime}}^{7}\right)$; again, this means that $L_{\theta}\left(\omega_{(n)}\right)=\omega_{(n)}$. In particular, this holds for the adjoint bundle associated to the adjoint representation on $s u(2)_{\mathbb{C}} \simeq \mathbb{C}^{3}$ (as complex representation spaces), from which we conclude that $\nabla_{0}^{(2)}$ coincides with $\left[\nabla_{0}, \cdot\right]$ (since this is the case if $\theta=0$ ).

\subsection{Twisted infinitesimal symmetries}

The noncommutative sphere $S_{\theta}^{4}$ can be realized as a quantum homogeneous space of the quantum orthogonal group $\mathrm{SO}_{\theta}(5)$ [36, 14, 1]. In other words, $\mathcal{A}\left(S_{\theta}^{4}\right)$ can be obtained as the subalgebra of $\mathcal{A}\left(\mathrm{SO}_{\theta}(5)\right)$ made of elements that are coinvariant under the natural coaction of $\mathrm{SO}_{\theta}(4)$ on $\mathrm{SO}_{\theta}(5)$. For our purposes, it turns out to be more convenient to take a dual point of view and consider an action instead of a coaction. We obtain a twisted symmetry action of the Lie algebra $s o(5)$ on $S_{\theta}^{4}$. Elements of $s o(5)$ act as twisted derivations on the algebra $\mathcal{A}\left(S_{\theta}^{4}\right)$. This action is lifted to $S_{\theta^{\prime}}^{7}$ and the basic instanton $\nabla_{0}$ described above is invariant under this infinitesimal twisted symmetry.

Different instantons are obtained by a twisted symmetry action of $s o(5,1)$. Classically, so $(5,1)$ is the conformal Lie algebra consisting of the infinitesimal diffeomorphisms leaving the conformal structure invariant. The Lie algebra $s o(5,1)$ is given by adding 5 generators to $s o(5)$. We explicitly describe its action on $S_{\theta}^{4}$ together with its lift to $S_{\theta^{\prime}}^{7}$ as an algebra of

\footnotetext{
${ }^{2}$ The Chern character classes and their realization as operators are in the Appendix.
} 
twisted derivations. The induced action on forms leaves the conformal structure invariant and when acting on $\nabla_{0}$ eventually results in a five-parameter family of instantons.

In fact, what we are really describing are Hopf algebras $\mathrm{U}_{\theta}(s o(5))$ and $\mathrm{U}_{\theta}(s o(5,1))$ which are obtained from the undeformed Hopf algebras $\mathrm{U}(s o(5))$ and $\mathrm{U}(s o(5,1))$ via a twist of a Drinfel'd type. Twisting of algebras and coalgebras has been known for some time [18, 19, 21]. The twists relevant for toric noncommutative manifolds are associated to the Cartan subalgebra of a Lie algebra and were already introduced in [31. Their use to implement symmetries of toric noncommutative manifolds like the ones of the present paper was made explicit in [35. The geometry of multi-parametric quantum groups and quantum enveloping algebras coming from twists has been studied in [2, 3].

Since we do not explicitly need Hopf algebras, we postpone a full fledged analysis of them and of their actions which will appear elsewhere. Here we shall rather give the explicit actions of both $s o(5)$ and $s o(5,1)$ as Lie algebras of twisted derivations (having however always in the back of our mind their origin as deformed Hopf algebras).

The eight roots of the Lie algebra so(5) are two-component vectors $r=\left(r_{1}, r_{2}\right)$ of the form $r=( \pm 1, \pm 1)$ and $r=(0, \pm 1), r=( \pm 1,0)$. There are corresponding generators $E_{r}$ of so(5) together with two mutually commuting generators $H_{1}, H_{2}$ of the Cartan subalgebra. The Lie brackets are

$$
\begin{aligned}
& {\left[H_{1}, H_{2}\right]=0, \quad\left[H_{j}, E_{r}\right]=r_{j} E_{r},} \\
& {\left[E_{-r}, E_{r}\right]=r_{1} H_{1}+r_{2} H_{2}, \quad\left[E_{r}, E_{r^{\prime}}\right]=N_{r, r^{\prime}} E_{r+r^{\prime}},}
\end{aligned}
$$

with $N_{r, r^{\prime}}=0$ if $r+r^{\prime}$ is not a root.

In order to give the action of $s o(5)$ on $S_{\theta}^{4}$, for convenience we introduce "partial derivatives", $\partial_{\mu}$ and $\partial_{\mu}^{*}$ with the usual action on the generators of the algebra $\mathcal{A}\left(S_{\theta}^{4}\right)$ i.e, $\partial_{\mu}\left(z_{\nu}\right)=\delta_{\mu \nu}, \partial_{\mu}\left(z_{\nu}^{*}\right)=0$, and $\partial_{\mu}^{*}\left(z_{\nu}^{*}\right)=\delta_{\mu \nu}, \partial_{\mu}^{*}\left(z_{\nu}\right)=0$. With these we construct operators on $\mathcal{A}\left(S_{\theta}^{4}\right)$,

$$
\begin{aligned}
H_{1} & =z_{1} \partial_{1}-z_{1}^{*} \partial_{1}^{*}, & H_{2} & =z_{2} \partial_{2}-z_{2}^{*} \partial_{2}^{*} \\
E_{+1,+1} & =z_{2} \partial_{1}^{*}-z_{1} \partial_{2}^{*}, & E_{+1,-1} & =z_{2}^{*} \partial_{1}^{*}-z_{1} \partial_{2} \\
E_{+1,0} & =\frac{1}{\sqrt{2}}\left(2 z_{0} \partial_{1}^{*}-z_{1} \partial_{0}\right), & E_{0,+1} & =\frac{1}{\sqrt{2}}\left(2 z_{0} \partial_{2}^{*}-z_{2} \partial_{0}\right),
\end{aligned}
$$

and $E_{-r}=\left(E_{r}\right)^{*}$, with the obvious meaning of the adjoint.

Proposition 20. The operators in (5.6) give a well defined action of so(5) on the algebra $\mathcal{A}\left(S_{\theta}^{4}\right)$ provided one extends them to the whole of $\mathcal{A}\left(S_{\theta}^{4}\right)$ as "twisted derivations" via the rules,

$$
\begin{aligned}
& E_{r}(a b)=E_{r}(a) \lambda^{\frac{1}{2}\left(-r_{1} H_{2}+r_{2} H_{1}\right)}(b)+\lambda^{\frac{1}{2}\left(r_{1} H_{2}-r_{2} H_{1}\right)}(a) E_{r}(b), \\
& H_{j}(a b)=H_{j}(a) b+a H_{j}(b)
\end{aligned}
$$

for any two elements $a, b \in \mathcal{A}\left(S_{\theta}^{4}\right)$; here $\lambda=e^{2 \pi \mathrm{i} \theta}$ is the deformation parameter.

Proof. With these twisted rules, one explicitly checks compatibility of the action (5.6) with the commutation relations (4.1) of $\mathcal{A}\left(S_{\theta}^{4}\right)$.

Remark 21. The operators $\lambda^{ \pm \frac{1}{2} r_{i} H_{j}}$ in (15.7) are understood as exponentials of diagonal matrices: on the generators $z_{\mu}$, $z_{\mu}^{*}$ of $\mathcal{A}\left(S_{\theta}^{4}\right)$, the operators $H_{1}$ and $H_{2}$ can be written as finite dimensional matrices. A comparison with equation (4.13) shows that $H_{1}$ and $H_{2}$ in (5.6) are the infinitesimal generators of the action of $\mathbb{T}^{2}$ on $S_{\theta}^{4}$. 
We can write the twisted action of $s o(5)$ on $\mathcal{A}\left(S_{\theta}^{4}\right)$ by using the quantization map $L_{\theta}$ introduced in Section 3. For $L_{\theta}(a) \in \mathcal{A}\left(S_{\theta}^{4}\right)$ and $t \in s o(5)$ a twisted action is defined by

$$
T \cdot L_{\theta}(a)=L_{\theta}(t \cdot a)
$$

where $T$ is the "quantization" of $t$ and $t \cdot a$ is the classical action of $\operatorname{so}(5)$ on $\mathcal{A}\left(S^{4}\right)$ (a better but heavier notation for the action $T \cdot$ would be $t \cdot \theta$ ). One checks that both of these definitions of the twisted action coincide. The latter definition allows one to define an action of $s o(5)$ on $C^{\infty}\left(S_{\theta}^{4}\right)$ by allowing $a$ to be in $C^{\infty}\left(S^{4}\right)$ in equation (5.8). Furthermore, as operators on the Hilbert space $\mathcal{H}$ of spinors, one could identify $\lambda^{\frac{1}{2}\left(r_{1} H_{2}-r_{2} H_{1}\right)}=U\left(\frac{1}{2} r \cdot \theta\right)$, with $r=\left(r_{1}, r_{2}\right), \theta$ the antisymmetric two by two matrix with $\theta_{12}=-\theta_{21}=\theta$ and $U(s)$ is the representation of $\mathbb{T}^{2}$ on $\mathcal{H}$ as in Sect 3.1 .

The twisted action of the Lie algebra $s o(5)$ on $\mathcal{A}\left(S_{\theta}^{4}\right)$ is extended to the differential calculus $\left(\Omega\left(S_{\theta}^{4}\right), \mathrm{d}\right)$ by requiring it to commute with the exterior derivative,

$$
T \cdot \mathrm{d} \omega:=\mathrm{d}(T \cdot \omega) .
$$

for $T \in$ so(5), $\omega \in \Omega\left(S_{\theta}^{4}\right)$. Then, we need to use the rule (5.7) on a generic form. For instance, on 1-forms we have,

$$
\begin{aligned}
& E_{r}\left(\sum_{k} a_{k} \mathrm{~d} b_{k}\right)=\sum_{k}\left(E_{r}\left(a_{k}\right) \mathrm{d}\left(\lambda^{\frac{1}{2}\left(-r_{1} H_{2}+r_{2} H_{1}\right)}\left(b_{k}\right)\right)+\lambda^{\frac{1}{2}\left(r_{1} H_{2}-r_{2} H_{1}\right)}\left(a_{k}\right) \mathrm{d}\left(E_{r}\left(b_{k}\right)\right)\right), \\
& H_{j}\left(\sum_{k} a_{k} \mathrm{~d} b_{k}\right)=\sum_{k}\left(H_{j}\left(a_{k}\right) \mathrm{d} b_{k}+a_{k} \mathrm{~d}\left(H_{j}\left(b_{k}\right)\right)\right) .
\end{aligned}
$$

The representation of $s o(5)$ on $S_{\theta}^{4}$ given in (5.6) is the fundamental vector representation. When lifted to $S_{\theta^{\prime}}^{7}$ one gets the fundamental spinor representation: as we see from the quadratic relations among corresponding generators, as given in (4.9), the lifting amounts to take the "square root" representation. The action of $\operatorname{so}(5)$ on $\mathcal{A}\left(S_{\theta^{\prime}}^{7}\right)$ is constructed by requiring twisted derivation properties via the rule (5.7) - when acting now on any two elements $a, b \in \mathcal{A}\left(S_{\theta}^{7}\right)$ - so as to reduce to the action (5.6) on $\mathcal{A}\left(S_{\theta}^{4}\right)$ when using the defining quadratic relations (4.9). The resulting action on $\mathcal{A}\left(S_{\theta^{\prime}}^{7}\right)$ can be given as the action of matrices $\Gamma$ 's on the $\psi$ 's,

$$
\psi_{a} \mapsto \sum_{b} \Gamma_{a b} \psi_{b}, \quad \psi_{a}^{*} \mapsto \sum_{b} \widetilde{\Gamma}_{a b} \psi_{b}^{*},
$$

with the matrices $\Gamma=\left\{H_{j}, E_{r}\right\}$ given explicitly by,

$$
\begin{aligned}
& H_{1}=\frac{1}{2}\left(\begin{array}{cccc}
1 & & & \\
& -1 & & \\
& & -1 & \\
& & & 1
\end{array}\right), \quad H_{2}=\frac{1}{2}\left(\begin{array}{cccc}
-1 & & & \\
& 1 & & \\
& & -1 & \\
& & & 1
\end{array}\right),
\end{aligned}
$$

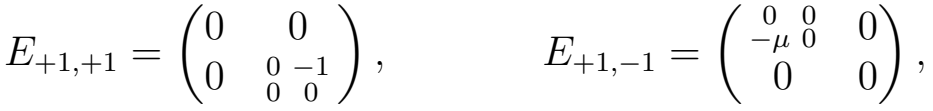

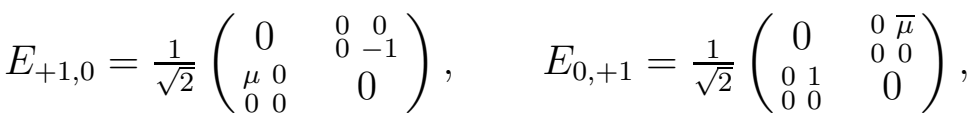

and $\widetilde{\Gamma}:=\sigma \Gamma \sigma^{-1}$ with

$$
\sigma:=\left(\begin{array}{ccc}
0 & -1 & 0 \\
1 & 0 & 0 \\
0 & 0 & -1
\end{array}\right)
$$


Furthermore, $E_{-r}=\left(E_{r}\right)^{*}$. With the twisted rules (5.7) for the action on products of any two elements $a, b \in \mathcal{A}\left(S_{\theta}^{7}\right)$, one checks compatibility of the above action with the commutation relations (4.4) of $\mathcal{A}\left(S_{\theta^{\prime}}^{7}\right)$. Again, the operators $\lambda^{ \pm \frac{1}{2} r_{i} H_{j}}$ in (5.7) are exponentials of diagonal matrices $H_{1}$ and $H_{2}$ given in the representation (5.11) and as above, one could think of $\lambda^{\frac{1}{2}\left(r_{1} H_{2}-r_{2} H_{1}\right)}$ as the operator $U\left(\frac{1}{2} r \cdot \theta\right)$.

Remark 22. Compare the form of the matrices $H_{1}$ and $H_{2}$ in the representation (5.11) above with the lifted action $\widetilde{\sigma}$ of $\widetilde{T}^{2}$ on $S_{\theta^{\prime}}^{7}$ as defined in (4.14). One checks that

$$
\widetilde{\sigma}_{s}=e^{\pi i\left(\left(s_{1}+s_{2}\right) H_{1}+\left(-s_{1}+s_{2}\right) H_{2}\right)},
$$

when acting on the spinor $\left(\psi_{1}, \cdots, \psi_{4}\right)$.

Notice that $\widetilde{\Gamma}=-\Gamma^{t}$ at $\theta=0$. There is a beautiful correspondence between the matrices in the representation (5.11) and the twisted Dirac matrices introduced in (4.11),

$$
\begin{array}{rlrl}
\frac{1}{4}\left[\gamma_{1}^{*}, \gamma_{1}\right] & =2 H_{1} & \frac{1}{4}\left[\gamma_{2}^{*}, \gamma_{2}\right]=2 H_{2} \\
\frac{1}{4}\left[\gamma_{1}, \gamma_{2}\right]=(\mu+\bar{\mu}) E_{+1,+1} & \frac{1}{4}\left[\gamma_{1}, \gamma_{2}^{*}\right]=(\mu+\bar{\mu}) E_{+1,-1} \\
\frac{1}{4}\left[\gamma_{1}, \gamma_{0}\right]=\sqrt{2} E_{+1,0} & \frac{1}{4}\left[\gamma_{2}, \gamma_{0}\right]=\sqrt{2} \bar{\mu} E_{0,+1} .
\end{array}
$$

Remark 23. It is straightforward to check that the twisted Dirac matrices satisfy the following relations under conjugation by $\sigma$ :

$$
\left(\sigma \gamma_{0} \sigma^{-1}\right)^{t}=\gamma_{0} ; \quad\left(\sigma \gamma_{1} \sigma^{-1}\right)^{t}=\gamma_{1} \lambda^{H_{2}} ; \quad\left(\sigma \gamma_{2} \sigma^{-1}\right)^{t}=\gamma_{2} \lambda^{H_{1}}
$$

As for $S_{\theta}^{4}$, the twisted action of $s o(5)$ on $\mathcal{A}\left(S_{\theta}^{7}\right)$ is straightforwardly extended to the differential calculus $\left(\Omega\left(S_{\theta}^{7}\right), \mathrm{d}\right)$. Furthermore, due to the form of $\widetilde{\Gamma}$ and the property $\Psi_{a 2}=\sigma_{a b} \psi_{b}^{*}$ for the second column of the matrix $\Psi$ in (4.6), we have also that so(5) acts on $\Psi$ by left matrix multiplication by $\Gamma$, and by right matrix multiplication on $\Psi^{*}$ by the matrix transpose $\widetilde{\Gamma}^{t}$ as follows

$$
\Psi_{a i} \mapsto \sum_{b} \Gamma_{a b} \Psi_{b i}, \quad \Psi_{i a}^{*} \mapsto \sum_{a} \Psi_{i b}^{*} \widetilde{\Gamma}_{a b}
$$

All this is used in the following

Proposition 24. The instanton gauge potential $\omega$ is invariant under the action of so(5).

Proof. From the above observations, the gauge potential transforms as,

$$
\omega=\Psi^{*} \mathrm{~d} \Psi \mapsto \Psi^{*}\left(\widetilde{\Gamma}^{t} \lambda^{-r_{1} H_{2}}+\lambda^{r_{2} H_{1}} \Gamma\right) \mathrm{d} \Psi .
$$

where $\lambda^{-r_{i} H_{j}}$ is understood in its representation (5.11) on $\mathcal{A}\left(S_{\theta}^{7}\right)$. Direct computation for $\Gamma=\left\{H_{j}, E_{r}\right\}$ shows that $\widetilde{\Gamma}^{t} \lambda^{-r_{1} H_{2}}+\lambda^{r_{2} H_{1}} \Gamma=0$, which finishes the proof. 


\subsection{Twisted conformal transformations}

In order to have new instantonic configurations we need to use conformal transformations. The conformal Lie algebra $s o(5,1)$ consists of the generators of $s o(5)$ together with the dilation and the so-called special conformal transformations. On $\mathbb{R}^{4}$ with coordinates $\left\{x_{\mu}, \mu=1, \ldots, 4\right\}$ they are given by the operators $H_{0}=\sum_{\mu} x_{\mu} \partial / \partial x_{\mu}$ and $G_{\mu}=2 x_{\mu} \sum_{\nu} x_{\nu} \partial / \partial x_{\nu}-\sum_{\nu} x_{\nu}^{2}\left(\partial / \partial x_{\nu}\right)$, respectively [28].

Since we are deforming with respect to the two-torus coming from the Cartan subalgebra $H_{1}, H_{2}$ of $s o(5)$, we write the Lie algebra $s o(5,1)$ as $s o(5)$ together with five extra generators, $H_{0}, G_{r}$, the latter labeled by the corresponding roots with respect to $H_{1}$ and $H_{2}$, that is $r=( \pm 1,0),(0, \pm 1)$. Besides the Lie brackets of $s o(5)$ given in (5.5), we have

$$
\begin{array}{lll}
{\left[H_{0}, H_{i}\right]=0,} & {\left[H_{j}, G_{r}\right]=r_{j} G_{r},} \\
{\left[H_{0}, G_{r}\right]=\sqrt{2} E_{r},} & {\left[H_{0}, E_{r}\right]=(\sqrt{2})^{-1} G_{r},}
\end{array}
$$

whenever $r=( \pm 1,0),(0, \pm 1)$, and

$$
\begin{array}{ll}
{\left[G_{-r}, G_{r}\right]=2 r_{1} H_{1}+2 r_{2} H_{2},} & {\left[G_{r}, G_{r^{\prime}}\right]=N_{r, r^{\prime}} E_{r+r^{\prime}},} \\
{\left[E_{r}, G_{r^{\prime}}\right]=\widetilde{N}_{r, r^{\prime}} G_{r+r^{\prime}},} & {\left[E_{-r}, G_{r}\right]=\sqrt{2} H_{0},}
\end{array}
$$

with, as before, $N_{r, r^{\prime}}=0$ if $r+r^{\prime}$ is not a root of $s o(5)$ and $\widetilde{N}_{r, r^{\prime}}=0$ if $r+r^{\prime}$ does not belong to $\{( \pm 1,0),(0, \pm 1)\}$.

The action of $s o(5,1)$ on $\mathcal{A}\left(S_{\theta}^{4}\right)$ is given by the operators (5.6) together with

$$
\begin{aligned}
& H_{0}=\partial_{0}-z_{0}\left(z_{0} \partial_{0}+z_{1} \partial_{1}+z_{1}^{*} \partial_{1}^{*}+z_{2} \partial_{2}+z_{2}^{*} \partial_{2}^{*}\right), \\
& G_{1,0}=2 \partial_{1}^{*}-z_{1}\left(z_{0} \partial_{0}+z_{1} \partial_{1}+z_{1}^{*} \partial_{1}^{*}+\bar{\lambda} z_{2} \partial_{2}+\lambda z_{2}^{*} \partial_{2}^{*}\right), \\
& G_{0,1}=2 \partial_{2}^{*}-z_{2}\left(z_{0} \partial_{0}+z_{1} \partial_{1}+z_{1}^{*} \partial_{1}^{*}+z_{2} \partial_{2}+z_{2}^{*} \partial_{2}^{*}\right),
\end{aligned}
$$

and $G_{-r}=\left(G_{r}\right)^{*}$. Note that the introduction of the extra $\lambda$ 's in $G_{1,0}$ (and $G_{-1,0}$ ) are necessary for the Lie algebra structure of $s o(5,1)$ - as dictated by the Lie brackets in (5.16) and (5.17) - to be preserved, that is in order to have a Lie algebra representation. Since the operators $H_{0}$ and $G_{r}$ are quadratic in the $z$ 's, one has to be careful when deriving the above Lie brackets and use the twisted rules (5.7). For instance, on the generator $z_{2}$, we have

$$
\begin{aligned}
{\left[E_{-1,-1}, G_{1,0}\right]\left(z_{2}\right) } & =E_{-1,-1}\left(-\bar{\lambda} z_{1} z_{2}\right)+G_{1,0}\left(z_{1}^{*}\right) \\
& =-\bar{\lambda}\left(E_{-1,-1}\left(z_{1}\right) \lambda^{H_{2}}\left(z_{2}\right)+\lambda^{H_{1}}\left(z_{1}\right) E_{-1,-1}\left(z_{2}\right)\right)+G_{1,0}\left(z_{1}^{*}\right) \\
& =-z_{2}^{*} z_{2}+z_{1} z_{1}^{*}+2-z_{1} z_{1}^{*}=G_{0,-1}\left(z_{2}\right)
\end{aligned}
$$

As for Proposition 20, a direct computation establishes the following,

Proposition 25. The operators in (5.6) and (5.18) give a well defined action of so $(5,1)$ on the algebra $\mathcal{A}\left(S_{\theta}^{4}\right)$ provided one extends them to the whole of $\mathcal{A}\left(S_{\theta}^{4}\right)$ as twisted derivations via the rules (5.7) together with

$$
\begin{aligned}
& G_{r}(a b)=G_{r}(a) \lambda^{\frac{1}{2}\left(-r_{1} H_{2}+r_{2} H_{1}\right)}(b)+\lambda^{\frac{1}{2}\left(r_{1} H_{2}-r_{2} H_{1}\right)}(a) G_{r}(b), \\
& H_{0}(a b)=H_{0}(a) b+a H_{0}(b)
\end{aligned}
$$

for any two elements $a, b \in \mathcal{A}\left(S_{\theta}^{4}\right)$. 
Equivalently, so $(5,1)$ could be defined to act on $\mathcal{A}\left(S_{\theta}^{4}\right)$ by

$$
T \cdot L_{\theta}(a)=L_{\theta}(t \cdot a)
$$

for $T$ the operator deforming $t \in s o(5,1)$ and $L_{\theta}(a) \in \mathcal{A}\left(S_{\theta}^{4}\right)$ deforming $a \in \mathcal{A}\left(S^{4}\right)$. Again, equation (5.20) makes sense for $a \in C^{\infty}\left(S^{4}\right)$, which defines an action of $s o(5,1)$ on $C^{\infty}\left(S_{\theta}^{4}\right)$. As before, the action on the differential calculus $\left(\Omega\left(S_{\theta}^{4}\right)\right.$, d) is obtained by requiring commutation with the exterior derivative: $T \cdot \mathrm{d} \omega=\mathrm{d}(T \cdot \omega)$, for $T \in \operatorname{so}(5,1)$ and $\omega \in \Omega\left(S_{\theta}^{4}\right)$. On products we shall have formulæ like the one in (5.9),

$$
\begin{aligned}
& G_{r}\left(\sum_{k} a_{k} \mathrm{~d} b_{k}\right)=\sum_{k}\left(G_{r}\left(a_{k}\right) \mathrm{d}\left(\lambda^{\frac{1}{2}\left(-r_{1} H_{2}+r_{2} H_{1}\right)}\left(b_{k}\right)\right)+\lambda^{\frac{1}{2}\left(r_{1} H_{2}-r_{2} H_{1}\right)}\left(a_{k}\right) \mathrm{d}\left(G_{r}\left(b_{k}\right)\right)\right), \\
& H_{0}\left(\sum_{k} a_{k} \mathrm{~d} b_{k}\right)=\sum_{k}\left(H_{0}\left(a_{k}\right) \mathrm{d} b_{k}+a_{k} \mathrm{~d}\left(H_{0}\left(b_{k}\right)\right)\right) .
\end{aligned}
$$

What we are dealing with are "infinitesimal" twisted conformal transformations:

Lemma 26. The Hodge $*_{\theta}$-structure of $\Omega\left(S_{\theta}^{4}\right)$ is invariant for the twisted action of so $(5,1)$,

$$
T \cdot\left(*_{\theta} \omega\right)=*_{\theta}(T \cdot \omega)
$$

Proof. Recall that $T\left(L_{\theta}(a)\right)=L_{\theta}(t \cdot a)$ for $a \in \mathcal{A}\left(S^{4}\right)$ and $T$ the "quantization" of $t \in$ so $(5,1)$. Then, since so $(5,1)$ leaves the Hodge $*$-structure of $\Omega\left(S^{4}\right)$ invariant and the differential d commutes with the action of $s o(5,1)$, if follows that the latter algebra leaves the Hodge $*_{\theta}$-structure of $\Omega\left(S_{\theta}^{4}\right)$ invariant as well.

Again, the action of $s o(5,1)$ on $S_{\theta}^{4}$ can be lifted to an action on $S_{\theta^{\prime}}^{7}$. And the latter action can be written as in (5.10) in terms of matrices $\Gamma$ 's acting on the $\psi$ 's,

$$
\psi_{a} \mapsto \sum_{b} \Gamma_{a b} \psi_{b}, \quad \psi_{a}^{*} \mapsto \sum_{b} \widetilde{\Gamma}_{a b} \psi_{b}^{*}
$$

where in addition to (5.11) we have also the matrices $\Gamma=\left\{H_{0}, G_{r}\right\}$, given explicitly by

$$
\begin{aligned}
H_{0} & =\frac{1}{2}\left(-z_{0} \mathbb{I}_{4}+\gamma_{0}\right), \\
G_{1,0} & =\frac{1}{2}\left(-z_{1} \lambda^{-H_{2}}+\gamma_{1}\right), \quad G_{0,1}=\frac{1}{2}\left(-z_{2}+\lambda^{-H_{1}} \gamma_{2}\right),
\end{aligned}
$$

with $G_{-r}=\left(G_{r}\right)^{*}$ and $\widetilde{\Gamma}=\sigma \Gamma \sigma^{-1}$. Notice the reappearance of the twisted Dirac matrices $\gamma_{\mu}, \gamma_{\mu}^{*}$ of (4.11) in the above expressions. In the above expressions, the operators $\lambda^{-H_{j}}$ are $4 \times 4$ matrices obtained from the spin representation (5.11) of $H_{1}$ and $H_{2}$ and given explicitily by

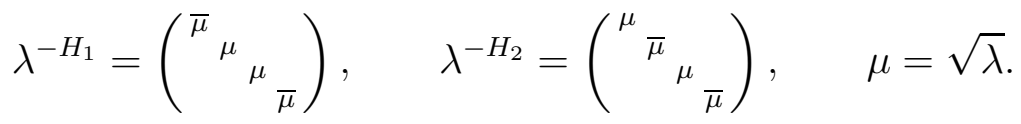

As for $s o(5)$, the action of $s o(5,1)$ on the matrix $\Psi$ is found to be by left matrix multiplication by $\Gamma$ and on $\Psi^{*}$ by $\widetilde{\Gamma}$,

$$
\Psi_{a i} \mapsto \sum_{b} \Gamma_{a b} \Psi_{b i}, \quad \Psi_{i a}^{*} \mapsto \sum_{a} \widetilde{\Gamma}_{a b} \Psi_{i b}^{*}
$$


Here we have to be careful with the ordering between $\widetilde{\Gamma}$ and $\Psi^{*}$ in the second term since the $\widetilde{\Gamma}$ 's involve the (not-central) $z$ 's. There are the following useful commutation relations between the $z_{\mu}$ 's and $\Psi$ :

$$
\begin{array}{ll}
z_{1} \Psi_{a i}=\left(\lambda^{-H_{2}}\right)_{a b} \Psi_{b i} z_{1}, & z_{2} \Psi_{a i}=\left(\lambda^{-H_{1}}\right)_{a b} \Psi_{b i} z_{2}, \\
z_{1} \Psi_{i a}^{*}=\Psi_{i b}^{*}\left(\lambda^{-H_{2}}\right)_{b a} z_{1}, & z_{2} \Psi_{i a}^{*}=\Psi_{i b}^{*}\left(\lambda^{-H_{1}}\right)_{b a} z_{2},
\end{array}
$$

with $\lambda^{-H_{j}}$ understood as the explicit matrices (5.24).

Proposition 27. The instanton gauge potential $\omega=\Psi^{*} \mathrm{~d} \Psi$ transforms under the action of the extra elements of $s o(5,1)$ as $\omega \mapsto \omega+\delta_{i} \omega$, where

$$
\begin{aligned}
& \delta_{0} \omega:=H_{0}(\omega)=-z_{0} \omega-\frac{1}{2} d z_{0} \mathbb{I}_{2}+\Psi^{*} \gamma_{0} \mathrm{~d} \Psi, \\
& \delta_{1} \omega:=G_{+1,0}(\omega)=-z_{1} \omega-\frac{1}{2} d z_{1} \mathbb{I}_{2}+\Psi^{*} \gamma_{1} \mathrm{~d} \Psi, \\
& \delta_{2} \omega:=G_{0,+1}(\omega)=-z_{2} \omega-\frac{1}{2} d z_{2} \mathbb{I}_{2}+\Psi^{*} \gamma_{2} \mathrm{~d} \Psi, \\
& \delta_{3} \omega:=G_{-1,0}(\omega)=-\omega \bar{z}_{1}-\frac{1}{2} d \bar{z}_{1} \mathbb{I}_{2}+\Psi^{*} \gamma_{1}^{*} \mathrm{~d} \Psi, \\
& \delta_{4} \omega:=G_{0,-1}(\omega)=-\omega \bar{z}_{2}-\frac{1}{2} d \bar{z}_{2} \mathbb{I}_{2}+\Psi^{*} \gamma_{2}^{*} \mathrm{~d} \Psi,
\end{aligned}
$$

with $\gamma_{\mu}, \gamma_{\mu}^{*}$ the twisted $4 \times 4$ Dirac matrices defined in (4.11).

Proof. The action of $H_{0}$ on the instanton gauge potential $\omega=\Psi^{*} \mathrm{~d} \Psi$ takes the form

$$
H_{0}(\omega)=H_{0}\left(\Psi^{*}\right) \mathrm{d} \Psi+\Psi^{*} \mathrm{~d}\left(H_{0}(\Psi)\right)=\Psi^{*}\left(-z_{0} \mathbb{I}_{4}+\gamma_{0}\right) \mathrm{d} \Psi-\frac{1}{2} d z_{0} \Psi^{*} \Psi
$$

since $z_{0}$ is central. Direct computation results in the above expression for $\delta_{0} \omega$. Instead, the twisted action of $G_{r}$ on $\omega$ takes the form,

$$
G_{r}: \omega_{i j} \mapsto \sum_{a, b, c} \widetilde{\Gamma}_{a b} \Psi_{i b}^{*}\left(\lambda^{-r_{1} H_{2}}\right)_{a c} \mathrm{~d} \Psi_{c j}+\left(\lambda^{r_{2} H_{1}}\right)_{a b} \Psi_{i b}^{*} \Gamma_{a c} \mathrm{~d} \Psi_{c j}+\left(\lambda^{r_{2} H_{1}}\right)_{a b} \Psi_{i b}^{*}\left(\mathrm{~d} \Gamma_{a c}\right) \Psi_{c j}
$$

where we used the fact that $\widetilde{H}_{j}=\sigma H_{j} \sigma^{-1}=-H_{j}$. Let us consider the case $r=(+1,0)$. Firstly, note that the complex numbers $\left(\lambda^{-H_{2}}\right)_{a c}$ commute with $\Psi_{i b}^{*}$ so that from the definition of $\Gamma$ and $\widetilde{\Gamma}$, and using (5.26), we obtain for the first two terms,

$$
-z_{1}\left(\Psi^{*} \mathrm{~d} \Psi\right)_{i j}+\frac{1}{2} \Psi_{i b}^{*}\left(\sigma \gamma_{1} \sigma^{-1}\right)_{c b}\left(\lambda^{-H_{2}}\right)_{c d} \mathrm{~d} \Psi_{d j}+\frac{1}{2} \Psi_{i b}^{*}\left(\gamma_{1}\right)_{b c} \mathrm{~d} \Psi_{c j} .
$$

The first term forms the matrix $-z_{1} \omega$ whereas the second two terms combine to give $\Psi^{*} \gamma_{1} \mathrm{~d} \Psi$, due to relation (5.14). Finally, using equation (5.26) the term $\Psi_{i b}^{*}\left(\mathrm{~d} \Gamma_{a c}\right) \Psi_{c j}$ reduces to $-\frac{1}{2} \mathrm{~d} z_{1} \Psi_{i b}^{*} \Psi_{b j}=-\frac{1}{2} \mathrm{~d} z_{1} \mathbb{I}_{2}$. The formulae for $r=(-1,0)$ and $r=(0, \pm 1)$ are established in likewise manner.

Remark 28. At first sight, the infinitesimal gauge potentials $\delta_{j} \omega$ given above do not seem to be su(2)-gauge potentials, in that they do not satisfy $\overline{\left(\delta_{j} \omega\right)_{k l}}=\left(\delta_{j} \omega\right)_{l k}$ and $\sum_{k}\left(\delta_{j} \omega\right)_{k k}=$ 0 . This is only due to the fact that the generators $G_{r}$ and $H_{0}$ are the deformed analogues of the generators of the complexified Lie algebra so $(5,1) \otimes_{\mathbb{R}} \mathbb{C}$. One recovers su(2)-gauge potentials by acting with the real generators $\frac{1}{2}\left(G_{r}+G_{r}^{*}\right), \frac{1}{2 i}\left(G_{r}-F_{r}^{*}\right)$ and $H_{0}$. The resulting gauge potentials, $\delta_{0} \omega, \frac{1}{2}\left(\delta_{1} \omega+\delta_{3} \omega\right), \frac{1}{2 i}\left(\delta_{1} \omega-\delta_{3} \omega\right), \frac{1}{2}\left(\delta_{2} \omega+\delta_{4} \omega\right)$ and $\frac{1}{2 i}\left(\delta_{2} \omega-\delta_{4} \omega\right)$, are traceless skew-hermitian matrices with entries in $\Omega^{1}\left(S_{\theta^{\prime}}^{7}\right)$. 
The transformations of the gauge potential $\omega$ under the twisted symmetry so $(5,1)$, given in Proposition 27, induce natural transformations of the canonical connection $\nabla_{0}$ in (5.1) to $\nabla_{t, i}:=\nabla_{0}+t \delta_{i} \omega+\mathcal{O}\left(t^{2}\right)$. We shall presently see that these new connections are (infinitesimal) instantons, i.e. their curvatures are self-dual. In fact, this also follows from Lemma 26 which states that $s o(5,1)$ acts by conformal transformation therefore leaving the self-duality equation $*_{\theta} F_{0}=F_{0}$ for the basic instanton $\nabla_{0}$ invariant.

We start by writing $\nabla_{t, i}$ in terms of the canonical connection on $\mathcal{E} \simeq p\left(\mathcal{A}\left(S_{\theta}^{4}\right)\right)^{4}$. Using the isomorphism, described in Example 14, between this module and the module of equivariant maps $\mathcal{A}\left(S_{\theta^{\prime}}^{7}\right) \bigotimes_{\rho} \mathbb{C}^{2}$, we find that $\nabla_{t, i}=p \mathrm{~d}+t \delta_{i} \alpha+\mathcal{O}\left(t^{2}\right)$ with

$$
\begin{array}{lll}
\delta_{0} \alpha=p \gamma_{0}(\mathrm{~d} p) p-\frac{1}{2} \Psi \mathrm{d} z_{0} \Psi^{*}, & \\
\delta_{1} \alpha=p \gamma_{1}(\mathrm{~d} p) p-\frac{1}{2} \Psi \mathrm{d} z_{1} \Psi^{*}, & & \delta_{3} \alpha=p \gamma_{1}^{*}(\mathrm{~d} p) p-\frac{1}{2} \Psi \mathrm{d} z_{1}^{*} \Psi^{*}, \\
\delta_{2} \alpha=p \gamma_{2}(\mathrm{~d} p) p-\frac{1}{2} \Psi \mathrm{d} z_{2} \Psi^{*}, & \delta_{4} \alpha=p \gamma_{2}^{*}(\mathrm{~d} p) p-\frac{1}{2} \Psi \mathrm{d} z_{2}^{*} \Psi^{*},
\end{array}
$$

The $\delta_{i} \alpha$ 's are $4 \times 4$ matrices with entries in the one-forms $\Omega^{1}\left(S_{\theta}^{4}\right)$ and satisfying conditions $p \delta_{i} \alpha=\delta_{i} \alpha p=p \delta_{i} \alpha p=\delta_{i} \alpha$, as expected from the general theory on connections on modules in Section 2, Indeed, using relations (5.26) one can move the $\mathrm{d} z_{\mu}$ 's to the left of $\Psi$ at the cost of some $\mu$ 's, so getting expression like $\mathrm{d} z_{i} p \in M_{4}\left(\Omega^{1}\left(S_{\theta}^{4}\right)\right)$.

From equation (2.10), the curvature $F_{t, i}$ of the connection $\nabla_{t, i}$ is given by

$$
F_{t, i}=F_{0}+\operatorname{tpd}\left(\delta_{i} \alpha\right)+\mathcal{O}\left(t^{2}\right) .
$$

In order to check self-duality (modulo $t^{2}$ ) of this curvature, we will express it in terms of the projection $p$ and consider $F_{t, i}$ as a two-form valued endomorphism on the module $\mathcal{E} \simeq p\left(\mathcal{A}\left(S_{\theta}^{4}\right)\right)^{4}$.

Proposition 29. The curvatures $F_{t, i}$ of the connections $\nabla_{t, i}, i=0, \ldots, 4$, are given by $F_{t, i}=F_{0}+t \delta_{i} F+\mathcal{O}\left(t^{2}\right)$, where $F_{0}=p \mathrm{~d} p \mathrm{~d} p$ and $\delta_{i} F$ are the following $4 \times 4$-matrices of 2-forms:

$$
\begin{array}{ll}
\delta_{0} F=-2 z_{0} F_{0}, & \\
\delta_{1} F=-2 z_{1} \lambda^{H_{2}} F_{0}, & \delta_{3} F=-2 z_{1}^{*} \lambda^{-H_{2}} F_{0}, \\
\delta_{2} F=-2 z_{2} \lambda^{H_{1}} F_{0} ; & \delta_{4} F=-2 z_{2}^{*} \lambda^{-H_{1}} F_{0} .
\end{array}
$$

Proof. A small computation yields for $\delta_{i} F=p \mathrm{~d}\left(\delta_{i} \alpha\right)$, thought of as an $\Omega^{2}\left(S_{\theta}^{4}\right)$-valued endomorphism on $\mathcal{E}$ the expression, $\delta_{i} F=p(\mathrm{~d} p) \gamma_{i}(\mathrm{~d} p) p-p \gamma_{i}(\mathrm{~d} p)(\mathrm{d} p) p$, with the notation $\gamma_{3}=\gamma_{1}^{*}$ and $\gamma_{4}=\gamma_{2}^{*}$, and using $p(\mathrm{~d} p) p=0$. Then, the crucial property $p\left(\mathrm{~d} p \gamma_{i}+\gamma_{i} \mathrm{~d} p\right)(\mathrm{d} p) p=0$ all $i=0, \ldots, 4$ yields $\delta_{i} F=-2 p \gamma_{i} \mathrm{~d} p \mathrm{~d} p p$. This is expressed as $\delta_{i} F=-2 p \gamma_{i} p d p d p$ by using $\mathrm{d} p=(\mathrm{d} p) p+p \mathrm{~d} p$. Finally, $p \gamma_{i} p=\Psi\left(\Psi^{*} \gamma_{i} \Psi\right) \Psi^{*}$, so that the result follows from the definition of the $z$ 's in terms of the Dirac matrices given in equation (4.10), together with the commutation relations between them and the matrix $\Psi$ in equation (5.26)

Proposition 30. The connections $\nabla_{t, i}$ are (infinitesimal) instantons, i.e.

$$
*_{\theta} F_{t, i}=F_{t, i} \quad \bmod t^{2} .
$$

Moreover, the connections $\nabla_{t, i}$ are not gauge equivalent to $\nabla_{0}$. 
Proof. The first point follows directly from the above expressions for $\delta_{i} F$ and the selfduality of $F_{0}$. To establish the gauge inequivalence of the connections $\nabla_{t, i}$ with $\nabla_{0}$, we recall that an infinitesimal gauge transformation is given by $\nabla_{0} \mapsto \nabla_{0}+t\left[\nabla_{0}, X\right]$ for $X \in \Gamma^{\infty}\left(\operatorname{ad}\left(S_{\theta^{\prime}}^{7}\right)\right)$. We need to show that $\delta_{i} \omega$ is orthogonal to $\left[\nabla_{0}, X\right]$ for any such $X$, i.e.

$$
\left(\left[\nabla_{0}, X\right], \delta_{i} \omega\right)_{2}=0
$$

with the natural inner product on $\Omega^{1}\left(\operatorname{ad}\left(S_{\theta^{\prime}}^{7}\right)\right):=\Omega^{1}\left(S_{\theta}^{4}\right) \otimes_{C^{\infty}\left(S_{\theta}^{4}\right)} \Gamma^{\infty}\left(\operatorname{ad}\left(S_{\theta^{\prime}}^{7}\right)\right)$. From Remark 19, it follows that

$$
\left(\nabla_{0}^{(2)}(X), \delta_{i} \omega\right)_{2}=\left(X,\left(\nabla_{0}^{(2)}\right)^{*}\left(\delta_{i} \omega\right)\right)_{2},
$$

which then should vanish for all $X$. From equation (5.20), we see that $\delta_{i} \omega=T_{i}(\omega)$ coincides with $L_{\theta}\left(t_{i} \cdot \omega^{(0)}\right)$ with $t_{i}$ and $\omega^{(0)}$ the classical counterparts of $T_{i}$ and $\omega$, respectively. In the undeformed case, the infinitesimal gauge potentials generated by acting with elements in $s o(5,1)-s o(5)$ on the basic instanton gauge potential $\omega^{(0)}$ satisfy $\left(\nabla_{0}^{(2)}\right)^{*}\left(\delta_{i} \omega^{(0)}\right)=0$ as shown in [6]. The result then follows from the observation that $\nabla_{0}^{(2)}$ commutes with the quantization map $L_{\theta}$ (cf. Remark 19).

\subsection{Local expressions}

In this section, we obtain "local expressions" for the instantons on $S_{\theta}^{4}$ constructed in the previous section; that is we map them to a noncommutative $\mathbb{R}_{\theta}^{4}$ obtained by "removing a point" from $S_{\theta}^{4}$. The algebra $\mathcal{A}\left(\mathbb{R}_{\theta}^{4}\right)$ of polynomial functions on the 4 -plane $\mathbb{R}_{\theta}^{4}$ is defined to be the $*$-algebra generated by elements $\zeta_{1}, \zeta_{2}$ satisfying

$$
\zeta_{1} \zeta_{2}=\lambda \zeta_{2} \zeta_{1} ; \quad \zeta_{1} \zeta_{2}^{*}=\bar{\lambda} \zeta_{2}^{*} \zeta_{1}
$$

with $\lambda=e^{2 \pi i \theta}$ as above. At $\theta=0$ one recovers the $*$-algebra $\mathcal{A}\left(\mathbb{R}^{4}\right)$ of polynomial functions on the usual 4-plane $\mathbb{R}^{4}$.

The algebra $\mathcal{A}\left(\mathbb{R}_{\theta}^{4}\right)$ can also be defined as the vector space $\mathcal{A}\left(\mathbb{R}^{4}\right)$ equipped with a deformed product $\times_{\theta}$ as in equation (3.2). Indeed, the torus $\mathbb{T}^{2}$ acts naturally on the two complex coordinates of $\mathbb{R}^{4} \simeq \mathbb{C}^{2}$. This also allows us to define the smooth algebra $C_{b}^{\infty}\left(\mathbb{R}_{\theta}^{4}\right)$ as the vector space $C_{b}^{\infty}\left(\mathbb{R}^{4}\right)$ of bounded smooth functions on $\mathbb{R}^{4}$ equipped with a deformed product $\times_{\theta}$. However, for our purposes it suffices to consider the polynomial algebra $\mathcal{A}\left(\mathbb{R}_{\theta}^{4}\right)$ with one self-adjoint central generator $\rho$ added together with relations $\rho^{2}\left(1+|\zeta|^{2}\right)=\left(1+|\zeta|^{2}\right) \rho^{2}=1$ where $|\zeta|^{2}:=\zeta_{1}^{*} \zeta_{1}+\zeta_{2}^{*} \zeta_{2}$ (this enlargement was already done in [14]). In the following, we will denote the enlarged algebra by $\widetilde{\mathcal{A}}\left(\mathbb{R}_{\theta}^{4}\right)$ and will also use the notation

$$
\rho^{2}=\left(1+|\zeta|^{2}\right)^{-1}=\frac{1}{1+|\zeta|^{2}} .
$$

Note that $\rho^{2}$ is an element in $C_{b}^{\infty}\left(\mathbb{R}_{\theta}^{4}\right)$.

One defines elements $\widetilde{z}_{\mu}, \mu=0,1,2$ in $\widetilde{\mathcal{A}}\left(\mathbb{R}^{4}\right)$ by

$$
\widetilde{z}_{0}=\left(1-|\zeta|^{2}\right)\left(1+|\zeta|^{2}\right)^{-1}, \quad \widetilde{z}_{j}=2 \zeta_{j}\left(1+|\zeta|^{2}\right)^{-1} \quad j=1,2,
$$

and checks that they satisfy the same relations as in (4.1) of the generators $z_{\mu}$ of $\mathcal{A}\left(S_{\theta}^{4}\right)$. The difference is that the classical point $z_{0}=-1, z_{j}=z_{j}^{*}=0$ of $S_{\theta}^{4}$ is not in the spectrum 
of $\widetilde{z}_{\mu}$. We interpret the noncommutative plane $\mathbb{R}_{\theta}^{4}$ as a "chart" of the noncommutative 4-sphere $S_{\theta}^{4}$ and equation (5.33) as the (inverse) stereographic projection from $S_{\theta}^{4}$ to $\mathbb{R}_{\theta}^{4}$. In fact, one can cover $S_{\theta}^{4}$ by two such charts with domain $\mathbb{R}_{\theta}^{4}$, and transition functions on $\mathbb{R}_{\theta}^{4} \backslash\{0\}$, where $\{0\}$ is the classical point $\zeta_{j}=\zeta_{j}^{*}=0$ of $\mathbb{R}_{\theta}^{4}$ (cf. [14 for more details).

A differential calculus $\left(\Omega\left(\mathbb{R}_{\theta}^{4}\right), \mathrm{d}\right)$ on $\mathbb{R}_{\theta}^{4}$ is obtained from the general procedure described in Section 3. Explicitly, $\Omega\left(\mathbb{R}_{\theta}^{4}\right)$ is the graded $*$-algebra generated by the elements $\zeta_{\mu}$ of degree 0 and $d \zeta_{\mu}$ of degree 1 with relations,

$$
\begin{aligned}
& d \zeta_{\mu} d \zeta_{\nu}+\lambda_{\mu \nu} d \zeta_{\nu} d \zeta_{\mu}=0, \quad d \zeta_{\mu} d \zeta_{\nu}^{*}+\lambda_{\nu \mu} d \zeta_{\nu}^{*} d \zeta_{\mu}=0 \\
& \zeta_{\mu} d \zeta_{\nu}=\lambda_{\mu \nu} d \zeta_{\nu} \zeta_{\mu}, \quad \zeta_{\mu} d \zeta_{\nu}^{*}=\lambda_{\nu \mu} d \zeta_{\nu}^{*} \zeta_{\mu}
\end{aligned}
$$

and $\lambda_{12}=\bar{\lambda}_{21}=: \lambda=e^{2 \pi i \theta}$. There is a unique differential $\mathrm{d}$ on $\Omega\left(\mathbb{R}_{\theta}^{4}\right)$ for which one has $\mathrm{d}: \zeta_{\mu} \mapsto d \zeta_{\mu}$ and a Hodge star operator $*_{\theta}: \Omega^{p}\left(\mathbb{R}_{\theta}^{4}\right) \rightarrow \Omega^{4-p}\left(\mathbb{R}_{\theta}^{4}\right)$, obtained from the classical Hodge star operator as before. In terms of the standard Riemannian metric on $\mathbb{R}^{4}$, on two-forms we have,

$$
*_{\theta} d \zeta_{1} d \zeta_{2}=-d \zeta_{1} d \zeta_{2}, \quad *_{\theta} d \zeta_{1} d \zeta_{1}^{*}=-d \zeta_{2} d \zeta_{2}^{*}, \quad *_{\theta} d \zeta_{1} d \zeta_{2}^{*}=d \zeta_{1} d \zeta_{2}^{*}
$$

and $*_{\theta}^{2}=\mathrm{id}$. These are the same formulae as the ones for the undeformed Hodge $*$ on $\mathbb{R}^{4}$ - since the metric is not changed in an isospectral deformation.

Again, we slightly enlarge the differential calculus $\Omega\left(\mathbb{R}_{\theta}^{4}\right)$ by adding the self-adjoint central generator $\rho$. The differential $\mathrm{d}$ on $\rho$ is derived from the Leibniz rule for $\mathrm{d}$ applied to its defining relation,

$$
\left(\mathrm{d} \rho^{2}\right)\left(1+|\zeta|^{2}\right)+\rho^{2} \mathrm{~d}\left(1+|\zeta|^{2}\right)=\mathrm{d}\left(\rho^{2}\left(1+|\zeta|^{2}\right)\right)=0
$$

so that $\rho \mathrm{d} \rho=\frac{1}{2} \mathrm{~d} \rho^{2}=-\frac{1}{2} \rho^{4} \mathrm{~d}\left(1+|\zeta|^{2}\right)=-\frac{1}{2} \rho^{4} \sum_{\mu}\left(\zeta_{\mu} d \zeta_{\mu}^{*}+\zeta_{\mu}^{*} d \zeta_{\mu}\right)$. The enlarged differential calculus will be denoted by $\widetilde{\Omega}\left(\mathbb{R}_{\theta}^{4}\right)$.

The stereographic projection from $S^{4}$ onto $\mathbb{R}^{4}$ is a conformal map commuting with the action of $\mathbb{T}^{2}$; thus it makes sense to investigate the form of the instanton connections on $S_{\theta}^{4}$ obtained in Proposition 27 on the local chart $\mathbb{R}_{\theta}^{4}$. As in [25], we first introduce a "local section" of the principal bundle $S_{\theta^{\prime}}^{7} \rightarrow S_{\theta}^{4}$ on the local chart of $S_{\theta}^{4}$ defined in (5.33). Let $u=\left(u_{1}, u_{2}\right)$ be a complex spinor of modulus one, $u_{1}^{*} u_{1}+u_{2}^{*} u_{2}=1$, and define

$$
\left(\begin{array}{l}
\psi_{1} \\
\psi_{2}
\end{array}\right)=\rho\left(\begin{array}{l}
u_{1} \\
u_{2}
\end{array}\right), \quad\left(\begin{array}{l}
\psi_{3} \\
\psi_{4}
\end{array}\right)=\rho\left(\begin{array}{cc}
\zeta_{1}^{*} & \zeta_{2}^{*} \\
-\mu \zeta_{2} & \bar{\mu} \zeta_{1}
\end{array}\right)\left(\begin{array}{l}
u_{1} \\
u_{2}
\end{array}\right)
$$

Remark 31. Strictly speaking, the symbols $\psi_{a}$ here denotes elements in the algebra $\mathcal{A}\left(S_{\theta^{\prime}}^{7}\right)$ enlarged by an extra generator which is the inverse of $1+z_{0}=2\left(1+\psi_{1}^{*} \psi_{1}+\psi_{2}^{*} \psi_{2}\right)$. Intuitively, this corresponds to "remove" the fiber $S^{3}$ in $S_{\theta^{\prime}}^{7}$ above the classical point $z_{0}=-1, z_{j}=z_{j}^{*}=0$ of the base space $S_{\theta}^{4}$.

The commutations rules of the $u_{j}$ 's with the $\zeta_{k}$ 's are dictated by those of the $\psi_{j}$ :

$$
u_{1} \zeta_{j}=\mu \zeta_{j} u_{1}, \quad u_{2} \zeta_{j}=\bar{\mu} \zeta_{j} u_{2}, \quad j=1,2 .
$$

The right action of $\mathrm{SU}(2)$ rotates the vector $u$ while mapping to the "same point" of $S_{\theta}^{4}$, which, using the definition (4.9), from the choice in (5.36) is found to be

$$
2\left(\psi_{1} \psi_{3}^{*}+\psi_{2}^{*} \psi_{4}\right)=\widetilde{z}_{1}, \quad 2\left(-\psi_{1}^{*} \psi_{4}+\psi_{2} \psi_{3}^{*}\right)=\widetilde{z}_{2}, \quad 2\left(\psi_{1}^{*} \psi_{1}+\psi_{2}^{*} \psi_{2}\right)-1=\widetilde{z}_{0},
$$


and is in the local chart (5.33), as expected.

By writing the unit vector $u$ as a matrix, $u=\left(\begin{array}{cc}u_{1} & -u_{2}^{*} \\ u_{2} & u_{1}^{*}\end{array}\right) \in \mathrm{SU}(2)$, we have

$$
\Psi=\rho\left(\begin{array}{cc}
\mathbb{I}_{2} & 0 \\
0 & \mathcal{Z}
\end{array}\right)\left(\begin{array}{l}
u \\
u
\end{array}\right), \quad \text { with } \mathcal{Z}=\left(\begin{array}{cc}
\zeta_{1}^{*} & \zeta_{2}^{*} \\
-\mu \zeta_{2} & \bar{\mu} \zeta_{1}
\end{array}\right) .
$$

Then, direct computation of the gauge potential $\omega=\Psi^{*} d \Psi$ yields

$$
\begin{aligned}
u \omega u^{*} & =\rho^{-1} \mathrm{~d} \rho+\rho^{2} \mathcal{Z}^{*} \mathrm{~d} \mathcal{Z}+(\mathrm{d} u) u^{*} \\
& =\frac{1}{\left(1+|\zeta|^{2}\right)}\left(\begin{array}{cc}
\sum_{i} \zeta_{i} d \zeta_{i}^{*}-d \zeta_{i} \zeta_{i}^{*} & 2\left(\zeta_{1} d \zeta_{2}^{*}-d \zeta_{1} \zeta_{2}^{*}\right) \\
2\left(\zeta_{2} d \zeta_{1}^{*}-d \zeta_{2} \zeta_{1}^{*}\right) & \sum_{i} \zeta_{i}^{*} d \zeta_{i}-d \zeta_{i}^{*} \zeta_{i}
\end{array}\right)+(\mathrm{d} u) u^{*}
\end{aligned}
$$

while its curvature $F_{0}=\mathrm{d} \omega+\omega^{2}$ is,

$$
u F_{0} u^{*}=\rho^{4} \mathrm{~d} \mathcal{Z}^{*} \mathrm{~d} \mathcal{Z}=\frac{1}{\left(1+|\zeta|^{2}\right)^{2}}\left(\begin{array}{cc}
d \zeta_{1} d \zeta_{1}^{*}-d \zeta_{2} d \zeta_{2}^{*} & 2 d \zeta_{1} d \zeta_{2}^{*} \\
2 d \zeta_{2} d \zeta_{1}^{*} & -d \zeta_{1} d \zeta_{1}^{*}-d \zeta_{2} d \zeta_{2}^{*}
\end{array}\right)
$$

From the expressions (5.35) for the Hodge operator on two forms, one checks that this curvature is self-dual, $*_{\theta}\left(u F_{0} u^{*}\right)=u F_{0} u^{*}$, as expected.

The explicit local expressions for the transformed - under infinitesimal conformal transformations - gauge potentials and their curvature can be obtained in a similar manner. As an example, let us work out the local expression for $\delta_{0} \omega$ which is the most transparent one. Given the expression for $\delta_{0} \omega$ in Proposition 27, a direct computation shows that its local counterpart is

$$
u \delta_{0} \omega u^{*}=-2 \rho \mathrm{d} \rho-2 \rho^{4} \mathcal{Z}^{*} \mathrm{~d} \mathcal{Z}
$$

giving for the transformed curvature,

$$
u F_{t, 0} u^{*}=F_{0}+2 t\left(1-2 \rho^{2}\right) F_{0}+\mathcal{O}\left(t^{2}\right) .
$$

It is clear that this rescaled curvature still satisfies the self-duality equation; this is also in concordance with Proposition $\left[29\right.$, being $\widetilde{z}_{0}=2 \rho^{2}-1$.

\subsection{Moduli space of instantons}

We will closely follow the infinitesimal construction of instantons for the undeformed case given in [6]. This will eventually result in the computation of the dimension of the "tangent space" to the moduli space of instantons on $S_{\theta}^{4}$ by index methods. It will turn out that the five-parameter family of instantons constructed in the previous section is indeed the complete set of infinitesimal instantons on $S_{\theta}^{4}$.

Let us start by considering a family of connections on $S_{\theta}^{4}$,

$$
\nabla_{t}=\nabla_{0}+t \alpha
$$

where $\alpha \in \Omega^{1}\left(\operatorname{ad}\left(S_{\theta^{\prime}}^{7}\right)\right) \equiv \Omega^{1}\left(S_{\theta}^{4}\right) \otimes_{C^{\infty}\left(S_{\theta}^{4}\right)} \Gamma^{\infty}\left(\operatorname{ad}\left(S_{\theta^{\prime}}^{7}\right)\right)$ and after Example 14 we have denoted $\Gamma^{\infty}\left(\operatorname{ad}\left(S_{\theta^{\prime}}^{7}\right)\right)=C^{\infty}\left(S_{\theta^{\prime}}^{7}\right) \otimes_{\text {ad }} s u(2)$. For $\nabla_{t}$ to be an instanton, we have to impose 
the self-duality equation $*_{\theta} F_{t}=F_{t}$ on the curvature $F_{t}=F_{0}+t[\nabla, \alpha]+\mathcal{O}\left(t^{2}\right)$ of $\nabla_{t}$. This leads, when differentiated with respect to $t$, at $t=0$, to the linearized self-duality equation

$$
P_{-}\left[\nabla_{0}, \alpha\right]=0,
$$

with $P_{-}:=\frac{1}{2}\left(1-*_{\theta}\right)$ the projection onto the antiself-dual 2 -forms. Here $\left[\nabla_{0}, \alpha\right]$,

$$
\left[\nabla_{0}, \alpha\right]_{i j}=\mathrm{d} \alpha_{i j}+\omega_{i k} \alpha_{k j}-\alpha_{i k} \omega_{k j}
$$

is an element in $\Omega^{2}\left(\operatorname{ad}\left(S_{\theta^{\prime}}^{7}\right)\right)$ and has vanishing trace, due to the fact that $\omega_{i k} \alpha_{k j}=\alpha_{k j} \omega_{i k}$ (cf. equations (5.1), (5.2) and the related discussion).

If the family $\nabla_{t}$ were obtained from an infinitesimal gauge transformation we would have had $\alpha=\left[\nabla_{0}, X\right]$, for some $X \in \Gamma^{\infty}\left(\operatorname{ad}\left(S_{\theta^{\prime}}^{7}\right)\right)$. Indeed, $\left[\nabla_{0}, X\right]$ is an element in $\Omega^{1}\left(\operatorname{ad}\left(S_{\theta^{\prime}}^{7}\right)\right)$ and $P_{-}\left[\nabla_{0},\left[\nabla_{0}, X\right]\right]=\left[P_{-} F_{0}, X\right]=0$, since $F_{0}$ is self-dual. Hence, we have defined an element in the first cohomology group $H^{1}$ of the so-called self-dual complex,

$$
0 \rightarrow \Omega^{0}\left(\operatorname{ad}\left(S_{\theta^{\prime}}^{7}\right)\right) \stackrel{\mathrm{d}_{0}}{\longrightarrow} \Omega^{1}\left(\operatorname{ad}\left(S_{\theta^{\prime}}^{7}\right)\right) \stackrel{\mathrm{d}_{1}}{\longrightarrow} \Omega_{-}^{2}\left(\operatorname{ad}\left(S_{\theta^{\prime}}^{7}\right)\right) \rightarrow 0
$$

where $\Omega^{0}\left(\operatorname{ad}\left(S_{\theta^{\prime}}^{7}\right)\right)=\Gamma^{\infty}\left(\operatorname{ad}\left(S_{\theta^{\prime}}^{7}\right)\right)$ and $\mathrm{d}_{0}=\left[\nabla_{0}, \cdot\right], \mathrm{d}_{1}:=P_{-}\left[\nabla_{0}, \cdot\right]$. Note that these operators are Fredholm operators, so that the cohomology groups of the complex are finite dimensional. As usual, the complex can be replaced by a single Fredholm operator

$$
\mathrm{d}_{0}^{*}+\mathrm{d}_{1}: \Omega^{1}\left(\operatorname{ad}\left(S_{\theta^{\prime}}^{7}\right)\right) \longrightarrow \Omega^{0}\left(\operatorname{ad}\left(S_{\theta^{\prime}}^{7}\right)\right) \oplus \Omega_{-}^{2}\left(\operatorname{ad}\left(S_{\theta^{\prime}}^{7}\right)\right)
$$

with $\mathrm{d}_{0}^{*}$ the adjoint of $\mathrm{d}_{0}$ with respect to the inner product (3.25).

Our goal is to compute $h^{1}=\operatorname{dim} H^{1}$, the number of "true" instantons. This is achieved by calculating the alternating sum $h^{0}-h^{1}+h^{2}$ of Betti numbers from the index of this Fredholm operator,

$$
\operatorname{index}\left(d_{0}^{*}+d_{1}\right)=-h^{0}+h^{1}-h^{2},
$$

while showing that $h^{0}=h^{2}=0$.

By definition, $H^{0}$ consists of the covariant constant elements in $\Gamma^{\infty}\left(\operatorname{ad}\left(S_{\theta^{\prime}}^{7}\right)\right)$. Since the operator $\left[\nabla_{0}, \cdot\right]$ commutes with the action of $\mathbb{T}^{2}$ and coincides with $\nabla_{0}^{(2)}$ on $\Gamma^{\infty}\left(\operatorname{ad}\left(S_{\theta^{\prime}}^{7}\right)\right)$ (cf. Remark 19), being covariantly constant means that

$$
\left[\nabla_{0}, X\right]=\nabla_{0}^{(2)}(X)=0 .
$$

If we write once more $X=L_{\theta}\left(X^{(0)}\right)$ in terms of its classical counterpart and use the fact that $\nabla_{0}^{(2)}$ commutes with $L_{\theta}$ (cf. Remark [19) we find that this condition entails

$$
\nabla_{0}^{(2)}\left(L_{\theta}\left(X^{(0)}\right)\right)=L_{\theta}\left(\nabla_{0}^{(2)}\left(X^{(0)}\right)\right)=0
$$

For the undeformed case, there are no covariant constant elements in $\Gamma^{\infty}\left(\operatorname{ad}\left(S^{7}\right)\right)$ for an irreducible self-dual connection on $\mathcal{E}$, thus we conclude that $h^{0}=0$. A completely analogous argument for the kernel of the operator $d_{1}^{*}$ shows that also $h^{2}=0$. 


\subsection{Dirac operator associated to the complex}

The next step consists in computing the index of the Fredholm operator $\mathrm{d}_{0}^{*}+\mathrm{d}_{1}$ defined in (5.48). Firstly, this operator can be replaced by a Dirac operator on the spinor bundle $\mathcal{S}$ with coefficients in the adjoint bundle. For this, we need the following lemma, which is a straightforward modification of its classical analogue [6]. Recall that the $\mathbb{Z}^{2}$-grading $\gamma_{5}$ induces a decomposition of the spinor bundle $\mathcal{S}=\mathcal{S}^{+} \oplus \mathcal{S}^{-}$. Note also that $\mathcal{S}^{-}$coincides classically with the charge -1 anti-instanton bundle. Indeed, the Levi-Civita connection when lifted to the spinor bundle and restricted to negative chirality spinors - has antiselfdual curvature. Similarly, $\mathcal{S}^{+}$coincides with the charge +1 instanton bundle. Then Remark 10 implies that the $C^{\infty}\left(S^{4}\right)$-modules $\Gamma^{\infty}\left(S^{4}, \mathcal{S}^{ \pm}\right)$have a module-basis that is homogeneous under the action of $\widetilde{\mathbb{T}}^{2}$. We conclude from $\widetilde{\mathbb{T}}^{2}$-equivariance that $\Gamma^{\infty}\left(S_{\theta}^{4}, \mathcal{S}^{-}\right)$ is isomorphic to the charge -1 anti-instanton bundle $\Gamma^{\infty}\left(S_{\theta^{\prime}}^{7} \times_{\mathrm{SU}(2)} \mathbb{C}^{2}\right)$ on $S_{\theta}^{4}$. Similarly $\Gamma^{\infty}\left(S_{\theta}^{4}, \mathcal{S}^{+}\right)$is isomorphic to the charge +1 instanton bundle.

Lemma 32. There are the following isomorphisms of right $C^{\infty}\left(S_{\theta}^{4}\right)$-modules,

$$
\begin{aligned}
\Omega^{1}\left(S_{\theta}^{4}\right) & \simeq \Gamma^{\infty}\left(S_{\theta}^{4}, \mathcal{S}^{+} \otimes \mathcal{S}^{-}\right) \simeq \Gamma^{\infty}\left(S_{\theta}^{4}, \mathcal{S}^{+}\right) \otimes_{C^{\infty}\left(S_{\theta}^{4}\right)} \Gamma^{\infty}\left(S_{\theta}^{4}, \mathcal{S}^{-}\right), \\
\Omega^{0}\left(S_{\theta}^{4}\right) \oplus \Omega_{-}^{2}\left(S_{\theta}^{4}\right) & \simeq \Gamma^{\infty}\left(S_{\theta}^{4}, \mathcal{S}^{-} \otimes \mathcal{S}^{-}\right) \simeq \Gamma^{\infty}\left(S_{\theta}^{4}, \mathcal{S}^{-}\right) \otimes_{C^{\infty}\left(S_{\theta}^{4}\right)} \Gamma^{\infty}\left(S_{\theta}^{4}, \mathcal{S}^{-}\right) .
\end{aligned}
$$

Proof. Since classically $\Omega^{1}\left(S^{4}\right) \simeq \Gamma^{\infty}\left(S^{4}, \mathcal{S}^{+} \otimes \mathcal{S}^{-}\right)$as $\sigma$-equivariant $C^{\infty}\left(S^{4}\right)$-bimodules, Lemma 5 shows that $\Omega^{1}\left(S_{\theta}^{4}\right) \simeq \Gamma^{\infty}\left(S_{\theta}^{4}, \mathcal{S}^{+} \otimes \mathcal{S}^{-}\right)$as $C^{\infty}\left(S_{\theta}^{4}\right)$-bimodules. The observations above the Lemma indicate that $\mathcal{S}^{ \pm} \simeq S^{7} \times{ }_{\rho^{ \pm}} \mathbb{C}^{2}$ for the spinor representation $\rho^{+} \oplus \rho^{-}$of $\operatorname{Spin}(4) \simeq \mathrm{SU}(2) \times \mathrm{SU}(2)$ on $\mathbb{C}^{4}$, so that

$$
\begin{aligned}
\Gamma^{\infty}\left(S_{\theta}^{4}, \mathcal{S}^{+} \otimes \mathcal{S}^{-}\right) & \simeq C^{\infty}\left(S_{\theta^{\prime}}^{7}\right) \otimes_{\rho^{+} \otimes \rho^{-}}\left(\mathbb{C}^{2} \otimes \mathbb{C}^{2}\right) \\
& \simeq\left(C^{\infty}\left(S_{\theta^{\prime}}^{7}\right) \bigotimes_{\rho^{+}} \mathbb{C}^{2}\right) \otimes_{C^{\infty}\left(S_{\theta}^{4}\right)}\left(C^{\infty}\left(S_{\theta^{\prime}}^{7}\right) \bigotimes_{\rho^{-}} \mathbb{C}^{2}\right),
\end{aligned}
$$

using Proposition 11 in the last line. This proves our claim. An analogous statement holds for the second isomorphism.

Let us forget for the moment the adjoint bundle $\operatorname{ad}\left(S_{\theta^{\prime}}^{7}\right)$. Since $\Omega\left(S_{\theta}^{4}\right) \simeq \Omega\left(S^{4}\right)$ as vector spaces and both $\mathrm{d}$ and the Hodge $*$ commute with the action of $\mathbb{T}^{2}$, the operator $\mathrm{d}^{*}+P_{-} \mathrm{d}$ can be understood as a map from $\Omega^{1}\left(S^{4}\right) \rightarrow \Omega^{0}\left(S^{4}\right) \oplus \Omega_{-}^{2}\left(S^{4}\right)$ (see Section 3.4). Under the isomorphisms of the above Lemma, this operator is replaced [6] by a Dirac operator with coefficients in $\mathcal{S}^{-}$,

$$
D^{\prime}: \Gamma^{\infty}\left(S_{\theta}^{4}, \mathcal{S}^{+} \otimes \mathcal{S}^{-}\right) \rightarrow \Gamma^{\infty}\left(S_{\theta}^{4}, \mathcal{S}^{-} \otimes \mathcal{S}^{-}\right)
$$

Twisting by the adjoint bundle ad $\left(S_{\theta^{\prime}}^{7}\right)$, merely results into a composition with the projection $p_{(2)}$ defining the bundle $\operatorname{ad}\left(S_{\theta^{\prime}}^{7}\right)$. Hence, eventually the operator $\mathrm{d}_{0}^{*}+\mathrm{d}_{1}$ is replaced by the Dirac operator

$$
\mathcal{D}: \Gamma^{\infty}\left(S_{\theta}^{4}, \mathcal{S}^{+} \otimes \mathcal{S}^{-} \otimes \operatorname{ad}\left(S_{\theta^{\prime}}^{7}\right)\right) \rightarrow \Gamma^{\infty}\left(S_{\theta}^{4}, \mathcal{S}^{-} \otimes \mathcal{S}^{-} \otimes \operatorname{ad}\left(S_{\theta^{\prime}}^{7}\right)\right)
$$

with coefficients in the vector bundle $\mathcal{S}^{-} \otimes \operatorname{ad}\left(S_{\theta^{\prime}}^{7}\right)$ on $S_{\theta}^{4}$.

We have finally arrived to the computation of the index of this Dirac operator which we do by means of the Connes-Moscovoci local index formula. It is given by the pairing,

$$
\operatorname{index}(\mathcal{D})=\left\langle\phi, \operatorname{ch}\left(\mathcal{S}^{-} \otimes \operatorname{ad}\left(S_{\theta^{\prime}}^{7}\right)\right)\right\rangle=\left\langle\phi, \operatorname{ch}\left(\mathcal{S}^{-}\right) \cdot \operatorname{ch}\left(\operatorname{ad}\left(S_{\theta^{\prime}}^{7}\right)\right)\right\rangle .
$$


In the Appendix we recall the expression for both the cyclic cocycle $\phi$ and the Chern characters, as well as their realization as operators $\pi_{D}(\operatorname{ch}(\cdot))$ on the the Hilbert space of spinors $\mathcal{H}$. In [27] we computed these operators for all modules associated to the principal bundle $S_{\theta^{\prime}}^{7} \rightarrow S_{\theta}^{4}$. In particular, for the adjoint bundle we found that

$$
\pi_{D}\left(\operatorname{ch}_{0}\left(\operatorname{ad}\left(S_{\theta^{\prime}}^{7}\right)\right)\right)=3, \quad \pi_{D}\left(\operatorname{ch}_{1}\left(\operatorname{ad}\left(S_{\theta^{\prime}}^{7}\right)\right)\right)=0, \quad \pi_{D}\left(\operatorname{ch}_{2}\left(\operatorname{ad}\left(S_{\theta^{\prime}}^{7}\right)\right)\right)=4\left(3 \gamma_{5}\right) .
$$

To compute the Chern character of the spinor bundle $\mathcal{S}^{-}$we use its mentioned identification with the charge -1 instanton bundle $\Gamma^{\infty}\left(S_{\theta^{\prime}}^{7} \times \times_{\mathrm{SU}(2)} \mathbb{C}^{2}\right)$ on $S_{\theta}^{4}$. It then follows from [15] (cf. also [27]) that

$$
\pi_{D}\left(\operatorname{ch}_{0}\left(\mathcal{S}^{-}\right)\right)=2, \quad \pi_{D}\left(\operatorname{ch}_{1}\left(\mathcal{S}^{-}\right)\right)=0, \quad \pi_{D}\left(\operatorname{ch}_{2}\left(\mathcal{S}^{-}\right)\right)=-3 \gamma_{5} .
$$

Combining both Chern characters and using the local index formula on $S_{\theta}^{4}$, we have

$$
\operatorname{index}(\mathcal{D})=6 \operatorname{Res}_{z=0} z^{-1} \operatorname{tr}\left(\gamma_{5}|D|^{-2 z}\right)+0+\frac{1}{2}(2 \cdot 4-3 \cdot 1) \operatorname{Res}_{z=0} \operatorname{tr}\left(3 \gamma_{5}^{2}|D|^{-4-2 z}\right),
$$

with $D$ identified with the classical Dirac operator on $S^{4}$ (recall that we do not change it in the isospectral deformation). Now, the first term vanishes due to index $(D)=0$ for this classical operator. On the other hand $\gamma_{5}^{2}=\mathbb{I}_{4}$, and

$$
3 \underset{z=0}{3 \operatorname{Res}} \operatorname{tr}\left(|D|^{-4-2 z}\right)=6 \operatorname{Tr}_{\omega}\left(|D|^{-4}\right)=2,
$$

since the Dixmier trace of $|D|^{-m}$ on the $m$-sphere equals $8 / m$ ! (cf. for instance [22, 24]). We conclude that index $(\mathcal{D})=5$ and for the moduli space of instantons on $S_{\theta}^{4}$, we have the following

Theorem 33. The tangent space at the base point $\nabla_{0}$ to the moduli space of (irreducible) $\mathrm{SU}(2)$-instantons on $S_{\theta}^{4}$ is five-dimensional.

\section{Towards Yang-Mills theory on $M_{\theta}$}

In this final Section, we shall briefly describe how the Yang-Mills theory on $S_{\theta}^{4}$ can be generalized to any compact four-dimensional toric noncommutative manifold $M_{\theta}$.

With $G$ a compact semisimple Lie group, let $P \rightarrow M$ be a principal $G$ bundle on $M$. We take $M$ to be a compact four-dimensional Riemannian manifold equipped with an isometrical action $\sigma$ of the torus $\mathbb{T}^{2}$. For the construction to work, we assume that this action can be lifted to an action $\widetilde{\sigma}$ of a cover $\widetilde{\mathbb{T}}^{2}$ on $P$ that commutes with the action of $G$. As in Section [3, we define the noncommutative algebras $C^{\infty}\left(P_{\theta}\right)$ and $C^{\infty}\left(M_{\theta}\right)$ as the vector spaces $C^{\infty}(P)$ and $C^{\infty}(M)$ with star products defined like in (3.2) with respect to the action of $\widetilde{\mathbb{T}}^{2}$ and $\mathbb{T}^{2}$ respectively or, equivalently as the images of $C^{\infty}(P)$ and $C^{\infty}(M)$ under the corresponding quantization map $L_{\theta}$. Since the action of $\widetilde{\mathbb{T}}^{2}$ is taken to commute with the action of $G$ on $P$, the action $\alpha$ of $G$ on the algebra $C^{\infty}(P)$ given by

$$
\alpha_{g}(f)(p)=f\left(g^{-1} \cdot p\right)
$$

induces an action of $G$ by automorphisms on the algebra $C^{\infty}\left(P_{\theta}\right)$. This also means that the inclusion $C^{\infty}(M) \subset C^{\infty}(P)$ as $G$-invariant elements in $C^{\infty}(P)$ extends to an inclusion $C^{\infty}\left(M_{\theta}\right) \subset C^{\infty}\left(P_{\theta}\right)$ of $G$-invariant element in $C^{\infty}\left(P_{\theta}\right)$. Clearly, the action of $G$ translates into a coaction of the Hopf algebra $C^{\infty}(G)$ on $C^{\infty}\left(P_{\theta}\right)$. 
Proposition 34. The inclusion $C^{\infty}\left(M_{\theta}\right) \hookrightarrow C^{\infty}\left(P_{\theta}\right)$ is a (principal) Hopf-Galois $C^{\infty}(G)$ extension.

Proof. One needs to establish bijectivity of the canonical map

$$
\begin{aligned}
\chi: C^{\infty}\left(P_{\theta}\right) \otimes_{C^{\infty}\left(M_{\theta}\right)} C^{\infty}\left(P_{\theta}\right) & \rightarrow C^{\infty}\left(P_{\theta}\right) \otimes C^{\infty}(G), \\
f^{\prime} \otimes_{C^{\infty}\left(M_{\theta}\right)} f & \mapsto f^{\prime} \Delta_{R}(f)=f^{\prime} f_{(0)} \otimes f_{(1)} .
\end{aligned}
$$

Now, for the undeformed case the bijectivity of the corresponding canonical map $\chi^{(0)}$ : $C^{\infty}(P) \otimes_{C^{\infty}(M)} C^{\infty}(P) \rightarrow C^{\infty}(P) \otimes C^{\infty}(G)$ follows by the very definition of a principal bundle. Furthermore, there is an isomorphism of vector spaces,

$$
\begin{aligned}
T: C^{\infty}\left(P_{\theta}\right) \otimes_{C^{\infty}\left(M_{\theta}\right)} C^{\infty}\left(P_{\theta}\right) & \rightarrow C^{\infty}(P) \otimes_{C^{\infty}(M)} C^{\infty}(P) \\
f^{\prime} \otimes_{C^{\infty}\left(M_{\theta}\right)} f & \mapsto \sum_{r} f_{r}^{\prime} \otimes_{C^{\infty}(M)} \widetilde{\sigma}_{r \theta}(f)
\end{aligned}
$$

where $f^{\prime}=\sum_{r} f_{r}^{\prime}$ is the homogeneous decomposition of $f^{\prime}$ under the action of $\widetilde{T}^{2}$. We claim that the canonical map is given as the composition $\chi=\left(L_{\theta} \otimes\right.$ id $) \circ \chi^{(0)} \circ T$; hence it is bijective. Indeed,

$$
\begin{aligned}
\left(L_{\theta} \otimes \mathrm{id}\right) \circ \chi^{(0)} \circ T\left(f^{\prime} \otimes_{C^{\infty}\left(M_{\theta}\right)} f\right) & =\sum_{r} L_{\theta}\left(f_{r}^{\prime} \widetilde{\sigma}_{r \theta}\left(f_{(0)}\right)\right) \otimes f_{(1)} \\
& =L_{\theta}\left(f^{\prime} \times_{\theta} f_{(0)}\right) \otimes f_{(1)}=\chi\left(f^{\prime} \otimes_{C^{\infty}\left(M_{\theta}\right)} f\right),
\end{aligned}
$$

since the action of $\widetilde{\mathbb{T}}^{2}$ on $C^{\infty}\left(P_{\theta}\right)$ commutes with the coaction of $C^{\infty}(G)$.

Noncommutative associated bundles are defined as in (4.15) by setting

$$
\mathcal{E}=C^{\infty}\left(P_{\theta}\right) \otimes_{\rho} W:=\left\{f \in C^{\infty}\left(P_{\theta}\right) \otimes W \mid\left(\alpha_{g} \otimes \mathrm{id}\right)(f)=\left(\mathrm{id} \otimes \rho(g)^{-1}\right)(f), \forall g \in G\right\}
$$

for a representation $\rho$ of $G$ on $W$. These $C^{\infty}\left(M_{\theta}\right)$ bimodules are finite projective since they are of the kind defined in Section 3.3 (cf. Remark 10). Moreover, Proposition 12 generalizes and reads $\operatorname{End}(\mathcal{E}) \simeq C^{\infty}\left(P_{\theta}\right) \bigotimes_{\text {ad }} L(W)$, where ad is the adjoint representation of $G$ on $L(W)$. Also, one identifies the adjoint bundle as the module coming from the adjoint representation of $G$ on $\mathfrak{g} \subset L(W)$, namely $\Gamma^{\infty}\left(\operatorname{ad}\left(P_{\theta}\right)\right):=C^{\infty}\left(P_{\theta}\right) \bigotimes_{a d} \mathfrak{g}$.

For a (right) finite projective $C^{\infty}\left(M_{\theta}\right)$-module $\mathcal{E}$ we define an inner product $(\cdot, \cdot)_{2}$ on $\operatorname{Hom}_{C^{\infty}\left(M_{\theta}\right)}\left(\mathcal{E}, \mathcal{E} \otimes_{C^{\infty}\left(M_{\theta}\right)} \Omega\left(M_{\theta}\right)\right)$ as in Section 4.3. The Yang-Mills action functional for a connection $\nabla$ on $\mathcal{E}$ in terms of its curvature $F$ is then defined by

$$
\mathrm{YM}(\nabla)=(F, F)_{2}
$$

This is a gauge invariant, positive and quartic functional. The derivation of the YangMills equations (4.32) on $S_{\theta}^{4}$ does not rely on the specific properties of $S_{\theta}^{4}$ and continues to hold on $M_{\theta}$. The same is true for the topological action, and $\operatorname{YM}(\nabla) \geq|\operatorname{Top}(\mathcal{E})|$ with equality iff $*_{\theta} F= \pm F$. In other words, instanton connections are minima of the Yang-Mills action.

The explicit construction of instanton connections on $S_{\theta}^{4}$ carried over in Section 5 can of course not be generalized to any manifold $M_{\theta}$. Local expressions could in principle 
be obtained on a "local chart" $\mathbb{R}_{\theta}^{4}$ of $M_{\theta}$ if $\mathbb{T}^{2}$ acts on the corresponding local chart $\mathbb{R}^{4}$ of $M$. On the other hand, the infinitesimal construction of instantons on $M_{\theta}$ giving the dimension of the "tangent of the moduli space" can be generalized to any toric noncommutative manifold $M_{\theta}$, again closely following [6]. It is essential however the existence of a "base point", i.e. an instanton connection that can be linearly perturbed to obtain a family of infinitesimal instantons.

\section{Acknowledgements}

We are grateful to an anonymous referee for an excellent review which led to a much improved version of the paper. We thank Paolo Aschieri and Marc Rieffel for useful remarks and suggestions. Part of the work was carried out while GL was visiting ESI in Vienna and INI in Cambridge. 


\section{A Local index formula}

Suppose in general that $(\mathcal{A}, \mathcal{H}, D, \gamma)$ is an even $p$-summable spectral triple with discrete simple dimension spectrum. For a projection $e \in M_{N}(A)$, the operator

$$
D_{e}=e\left(D \otimes \mathbb{I}_{N}\right) e
$$

is a Fredholm operator, thought of as the Dirac operator with coefficient in the module determined by $e$. The local index formula of Connes and Moscovici [16] provides a method to compute its index via the pairing of suitable cyclic cycles and cocycles. We shall recall the "even" case since it is the one that is relevant for the present paper.

Let $C_{*}(\mathcal{A})$ be the chain complex over the algebra $\mathcal{A}$; in degree $n, C_{n}(\mathcal{A}):=\mathcal{A}^{\otimes(n+1)}$. On this complex there are defined the Hochschild operator $b: C_{n}(\mathcal{A}) \rightarrow C_{n-1}(\mathcal{A})$ and the boundary operator $B: C_{n}(\mathcal{A}) \rightarrow C_{n+1}(\mathcal{A})$, satisfying $b^{2}=0, B^{2}=0, b B+B b=0$; thus $(b+B)^{2}=0$. From general homological theory, one defines a bicomplex $C C_{*}(\mathcal{A})$ by $C C_{(n, m)}(\mathcal{A}):=C C_{n-m}(\mathcal{A})$ in bi-degree $(n, m)$. Dually, one defines $C C^{*}(\mathcal{A})$ as functionals on $C C_{*}(\mathcal{A})$, equipped with the dual Hochschild operator $b$ and coboundary operator $B$ (we refer to [12] and [29] for more details on this).

Theorem 35 (Connes-Moscovici [16]).

(a) An even cocycle $\phi^{*}=\sum_{k \geq 0} \phi^{2 k}$ in $C C^{*}(\mathcal{A}),(b+B) \phi^{*}=0$, is defined by the following formula. For $k=0$,

$$
\phi^{0}(a):=\operatorname{Res}_{z=0} z^{-1} \operatorname{tr}\left(\gamma a|D|^{-2 z}\right)
$$

whereas for $k \neq 0$

$$
\phi^{2 k}\left(a^{0}, \ldots, a^{2 k}\right):=\sum_{\alpha} c_{k, \alpha} \operatorname{Res}_{z=0} \operatorname{tr}\left(\gamma a^{0}\left[D, a^{1}\right]^{\left(\alpha_{1}\right)} \cdots\left[D, a^{2 k}\right]^{\left(\alpha_{2 k}\right)}|D|^{-2(|\alpha|+k+z)}\right),
$$

with

$$
c_{k, \alpha}=(-1)^{|\alpha|} \Gamma(k+|\alpha|)\left(\alpha !\left(\alpha_{1}+1\right)\left(\alpha_{1}+\alpha_{2}+2\right) \cdots\left(\alpha_{1}+\cdots+\alpha_{2 k}+2 k\right)\right)^{-1}
$$

and $T^{(j)}$ denotes the $j^{\prime}$ th iteration of the derivation $T \mapsto\left[D^{2}, T\right]$.

(b) For $e \in K_{0}(\mathcal{A})$, the Chern character $\operatorname{ch}_{*}(e)=\sum_{k \geq 0} \operatorname{ch}_{k}(e)$ is the even cycle in $C C_{*}(\mathcal{A}),(b+B) \operatorname{ch}_{*}(e)=0$, defined by the following formula. For $k=0$,

$$
\operatorname{ch}_{0}(e):=\operatorname{tr}(e)
$$

whereas for $k \neq 0$

$$
\operatorname{ch}_{k}(e):=(-1)^{k} \frac{(2 k) !}{k !} \sum\left(e_{i_{0} i_{1}}-\frac{1}{2} \delta_{i_{0} i_{1}}\right) \otimes e_{i_{1} i_{2}} \otimes e_{i_{2} i_{3}} \otimes \cdots \otimes e_{i_{2 k} i_{0}} .
$$

(c) The index of the operator $D_{e}$ is given by the natural pairing between cycles and cocycles,

$$
\operatorname{index} D_{e}=\left\langle\phi^{*}, \operatorname{ch}_{*}(e)\right\rangle \text {. }
$$


For toric noncommutative manifolds, the above local index formula simplifies drastically [27].

Theorem 36. For a projection $p \in M_{N}\left(C^{\infty}\left(M_{\theta}\right)\right)$, we have

$$
\operatorname{index} D_{p}=\operatorname{Res}_{z=0} z^{-1} \operatorname{tr}\left(\gamma p|D|^{-2 z}\right)+\sum_{k \geq 1} \frac{(-1)^{k}}{k} \operatorname{Res}_{z=0} \operatorname{tr}\left(\gamma\left(p-\frac{1}{2}\right)[D, p]^{2 k}|D|^{-2(k+z)}\right)
$$

and now the trace tr comprises a matrix trace as well.

Proof. Recall that the quantization map $L_{\theta}$ preserves the spectral decomposition, for the toric action of $\widetilde{\mathbb{T}}^{n}$, of smooth operators (see equation (3.7)). Then, once extended the deformed $\times_{\theta}$-product to $C^{\infty}\left(M_{\theta}\right) \bigcup\left[D, C^{\infty}\left(M_{\theta}\right)\right]$ - which can be done unambiguously since $D$ is of degree 0 - we write the local cocycles $\phi^{2 k}$ in Theorem 35 in terms of the quantization map $L_{\theta}$ :

$$
\begin{aligned}
\phi^{2 k}\left(L_{\theta}\left(f^{0}\right)\right. & \left., L_{\theta}\left(f^{1}\right), \ldots, L_{\theta}\left(f^{2 k}\right)\right) \\
& =\operatorname{Res}_{z=0} \operatorname{tr}\left(\gamma L_{\theta}\left(f^{0} \times_{\theta}\left[D, f^{1}\right]^{\left(\alpha_{1}\right)} \times_{\theta} \cdots \times_{\theta}\left[D, f^{2 k}\right]^{\left(\alpha_{2 k}\right)}\right)|D|^{-2(|\alpha|+k+z)}\right) .
\end{aligned}
$$

Suppose now that $f^{0}, \ldots, f^{2 k} \in C^{\infty}(M)$ are homogeneous of degree $r^{0}, \ldots, r^{2 k}$ respectively, so that the operator $f^{0} \times_{\theta}\left[D, f^{1}\right]^{\left(\alpha_{1}\right)} \times_{\theta} \cdots \times_{\theta}\left[D, f^{2 k}\right]$ is a homogeneous element of degree $r=\sum_{j=0}^{2 k} r^{j}$. It is in fact a multiple of $f^{0}\left[D, f^{1}\right] \cdots\left[D, f^{2 k}\right]$ as can be established by working out the $\times_{\theta}$-products. Forgetting about this factor - which is a power of the deformation parameter $\lambda$ - we obtain from (3.6) that

$$
L_{\theta}\left(f^{0} \times_{\theta}\left[D, f^{1}\right]^{\left(\alpha_{1}\right)} \times_{\theta} \cdots \times_{\theta}\left[D, f^{2 k}\right]\right)=f^{0}\left[D, f^{1}\right] \cdots\left[D, f^{2 k}\right] U\left(\frac{1}{2} r \cdot \theta\right) .
$$

Each term in the local index formula for $\left(C^{\infty}\left(M_{\theta}\right), \mathcal{H}, D\right)$ then takes the form

$$
\operatorname{Res}_{z=0} \operatorname{tr}\left(\gamma f^{0}\left[D, f^{1}\right]^{\left(\alpha_{1}\right)} \cdots\left[D, f^{2 k}\right]^{\left(\alpha_{2 k}\right)}|D|^{-2(|\alpha|+k+z)} U(s)\right)
$$

for $s=\frac{1}{2} r \cdot \theta \in \widetilde{\mathbb{T}}^{n}$. The appearance of the operator $U(s)$ here is a consequence of the close relation with the index formula for $\mathbb{T}^{n}$-equivariant Dirac spectral triples. In [9] an even dimensional compact spin manifold $M$ on which a (connected compact) Lie group $G$ acts by isometries was studied. The equivariant Chern character was defined as an equivariant version of the JLO-cocycle, the latter being an element in equivariant entire cyclic cohomology. The essential point is that an explicit formula for the above residues was obtained. In the case of a $\mathbb{T}^{n}$-action on $M$ this is

$$
\begin{aligned}
\operatorname{Res}_{z=0} \operatorname{tr}\left(\gamma f^{0}\left[D, f^{1}\right]^{\left(\alpha_{1}\right)} \cdots\left[D, f^{2 k}\right]^{\left(\alpha_{2 k}\right)}|D|^{-2(|\alpha|+k+z)} U(s)\right) \\
=\Gamma(|\alpha|+k) \lim _{t \rightarrow 0} t^{|\alpha|+k} \operatorname{tr}\left(\gamma f^{0}\left[D, f^{1}\right]^{\left(\alpha_{1}\right)} \cdots\left[D, f^{2 k}\right]^{\left(\alpha_{2 k}\right)} e^{-t D^{2}} U(s)\right),
\end{aligned}
$$

for every $s \in \widetilde{\mathbb{T}}^{n}$. Moreover, from Thm 2 in $[9]$ the limit vanishes when $|\alpha| \neq 0$. This finishes the proof of our theorem.

By inserting the symbol $\pi$ for the algebra representation, the components of the Chern character are represented as operators on the Hilbert space $\mathcal{H}$ by explicit formulæ,

$$
\pi_{D}\left(\operatorname{ch}_{k}(e)\right):=(-1)^{k} \frac{(2 k) !}{k !} \sum\left(\pi\left(e_{i_{0} i_{1}}\right)-\frac{1}{2} \delta_{i_{0} i_{1}}\right)\left[D, \pi\left(e_{i_{1} i_{2}}\right)\right]\left[D, \pi\left(e_{i_{1} i_{2}}\right)\right] \cdots\left[D, \pi\left(e_{i_{2 k} i_{0}}\right)\right]
$$

for $k>0$, while $\pi_{D}\left(\operatorname{ch}_{0}(e)\right)=\sum \pi\left(e_{i_{0} i_{0}}\right)$. 


\section{References}

[1] P. Aschieri and F. Bonechi. On the noncommutative geometry of twisted spheres. Lett. Math. Phys. 59 (2002) 133-156.

[2] P. Aschieri and L. Castellani. Bicovariant Calculus on Twisted $I S O(N)$, Quantum Poincarè Group and Quantum Minkowski Space. Int. J. Mod. Phys. A11 (1996) 4513-4549.

[3] P. Aschieri and L. Castellani. Universal Enveloping Algebra and Differential Calculi on Inhomogeneous Orthogonal q-groups. J. Geom. Phys. 26 (1998) 247-271.

[4] M. F. Atiyah. The Geometry of Yang-Mills Fields. Fermi Lectures, Scuola Normale Pisa, 1979.

[5] M. F. Atiyah, N. J. Hitchin, V. G. Drinfel'd and Yu. I. Manin. Construction of instantons. Phys. Lett. A65 (1978) 185-187.

[6] M. F. Atiyah, N. J. Hitchin, and I. M. Singer. Self-duality in four-dimensional Riemannian geometry. Proc. Roy. Soc. Lond. A362 (1978) 425-461.

[7] A. Belavin, A. Polyakov, A. Schwarz, Y. Tyupkin. Pseudoparticle solutions of the Yang-Mills equations, Phys. Lett. 59B (1975) 85-87.

[8] V. Chari and A. Pressley. A guide to quantum groups. Cambridge University Press, Cambridge, 1994.

[9] S. Chern and X. Hu. Equivariant Chern character for the invariant Dirac operator. Michigan Math. J. 44 (1997) 451-473.

[10] A. Connes. $C^{*}$-algèbres et géométrie differentielle. C.R. Acad. Sci. Paris Ser. A-B, 290 (1980) A599-A604.

[11] A. Connes. Noncommutative differential geometry. IHES Sci. Publ. Math. 62 (1985) $257-360$.

[12] A. Connes. Noncommutative Geometry. Academic Press, San Diego, 1994.

[13] A. Connes. Gravity coupled with matter and the foundation of noncommutative geometry. Commun. Math. Phys. 182 (1996) 155-176.

[14] A. Connes and M. Dubois-Violette. Noncommutative finite-dimensional manifolds. I. Spherical manifolds and related examples. Commun. Math. Phys. 230 (2002) 539-579.

[15] A. Connes and G. Landi. Noncommutative manifolds: The instanton algebra and isospectral deformations. Commun. Math. Phys. 221 (2001) 141-159.

[16] A. Connes and H. Moscovici. The local index formula in noncommutative geometry. Geom. Funct. Anal. 5 (1995) 174-243. 
[17] J. Dixmier. Existence de traces non normales. C.R. Acad. Sci. Paris Sér A-B, 262 (1966) A1107-A1108.

[18] V.G. Drinfel'd. Constant quasiclassical solutions of the Yang-Baxter quantum equation. Soviet Math. Dokl. 28 (1983) 667-671.

[19] V.G. Drinfel'd. Quasi-Hopf algebras. Leningrad Math. J. 1 (1990) 1419-1457.

[20] V. Gayral, B. Iochum, and J. C. Várilly. Dixmier traces on noncompact isospectral deformations. J. Funct. Anal. 237 (2006) 507-539.

[21] A. Giaquinto, J.J. Zhang. Bialgebra actions, twists and universal deformation formulas. J. Pure Appl. Algebra. 128 (1998) 133-151.

[22] J. M. Gracia-Bondía, J. C. Várilly, and H. Figueroa. Elements of Noncommutative Geometry. Birkhäuser, Boston, 2001.

[23] P. Julg. K-théorie équivariante et produits croisés. C.R. Acad. Sci. Paris, 292 (1981) 629-632.

[24] G. Landi. An Introduction to Noncommutative Spaces and their Geometry. SpringerVerlag, Berlin, 1997.

[25] G. Landi. Spin-Hall effect with quantum group symmetry. Lett. Math. Phys. 75 (2006) 187-200.

[26] G. Landi, C. Pagani, C. Reina, and W. van Suijlekom. work in progress.

[27] G. Landi and W. van Suijlekom. Principal fibrations from noncommutative spheres. Commun. Math. Phys. 260 (2005) 203-225.

[28] S. Lie. Theorie der transformationsgruppen. Chelsea, New York, 1970.

[29] J.-L. Loday. Cyclic Homology. Springer-Verlag, Berlin, 1992.

[30] N. Nekrasov, A. Schwarz. Instantons on noncommutative $R^{4}$, and $(2,0)$ superconformal six dimensional theory. Commun. Math. Phys. 198 (1998) 689-703.

[31] N. Reshetikhin. Multiparameter quantum groups and twisted quasitriangular Hopf algebras. Lett. Math. Phys. 20 (1990) 331-335.

[32] M. A. Rieffel. Non-commutative tori - A case study of non-commutative differentiable manifolds. Contemp. Math. 105 (1990) 191-212.

[33] M.A. Rieffel. Deformation Quantization for Actions of $\mathbb{R}^{d}$. Memoirs of the Amer. Math. Soc. 506, Providence, RI, 1993.

[34] M. A. Rieffel. $K$-groups of $C^{*}$-algebras deformed by actions of $R^{d}$. J. Funct. Anal. 116 (1993) 199-214.

[35] A. Sitarz. Twists and spectral triples for isospectral deformations. Lett. Math. Phys. 58 (2001) 69-79. 
[36] J. C. Várilly. Quantum symmetry groups of noncommutative spheres. Commun. Math. Phys. 221 (2001) 511-523. 